\title{
Scaling Limits for Non-intersecting Polymers and Whittaker Measures
}

\author{
Samuel G. G. Johnston ${ }^{1} \cdot$ Neil O'Connell $^{2}$ (1) \\ Received: 3 November 2019 / Accepted: 19 March 2020 / Published online: 1 April 2020 \\ (c) The Author(s) 2020
}

\begin{abstract}
We study the partition functions associated with non-intersecting polymers in a random environment. By considering paths in series and in parallel, the partition functions carry natural notions of subadditivity, allowing the effective study of their asymptotics. For a certain choice of random environment, the geometric RSK correspondence provides an explicit representation of the partition functions in terms of a stochastic interface. Formally this leads to a variational description of the macroscopic behaviour of the interface and hence the free energy of the associated non-intersecting polymer model. At zero temperature we relate this variational description to the Marčenko-Pastur distribution, and give a new derivation of the surface tension of the bead model.
\end{abstract}

Keywords Non-intersecting paths $\cdot$ Polymers $\cdot$ Whittaker measures $\cdot$ Stochastic interfaces

\section{Introduction and Summary}

We study the partition functions associated with a natural model for non-intersecting polymers in a random environment. Apart from being an interesting physical model in its own right, this model is motivated by recent developments on connections between random polymers and Whittaker functions, obtained via the geometric RSK correspondence [14,37]. By considering paths in series and in parallel, the partition functions carry natural notions of subadditivity allowing the effective study of their asymptotics. We use this subadditivity to show that for a small number of paths, the free energy has a linear dependence on the number of paths (Theorem 2.2). Interestingly, it seems quite difficult to prove this using the integrable structure of the log-gamma polymer model; instead we give a general argument, valid for general weights. We also determine the correct order of scaling for the partition function associated with a large number of paths (see (2.15), (2.16) and Corollary 2.8).

Communicated by Ivan Corwin.

Samuel G. G. Johnston

sggjohnston@gmail.com

1 Institut für Mathematik und Wissenschaftliches Rechnen, University of Graz, Graz 8010, Austria

2 School of Mathematics and Statistics, University College Dublin, Dublin 4, Ireland 
We then turn our discussion to the random polymer model with log-gamma weights, where the geometric RSK correspondence provides an explicit representation of the partition functions in terms of a stochastic interface [14,19]. Formally this leads to a variational description of the macroscopic behaviour of the interface and hence the free energy of the associated non-intersecting polymer model. At zero temperature we relate this variational description to the Marčenko-Pastur distribution, and give a new derivation of the surface tension of the bead model, which was recently computed by Sun [43] using very different methods. In the remainder of Sect. 1, we summarise the main results of the paper, beginning with some preliminary definitions.

\subsection{Preliminary Definitions}

Let $\mathbf{e}_{1}=(1,0)$ and $\mathbf{e}_{2}=(0,1)$ be the standard basis for $\mathbb{Z}^{2}$. For points $x$ and $y$ in $\mathbb{Z}^{2}$, a path $\pi$ from $x$ to $y$ is a set of points $\pi=\left\{a_{0}, \ldots, a_{p}\right\}$ in $\mathbb{Z}^{2}$ such that $a_{0}=x, a_{p}=y$, and $a_{j+1}-a_{j}$ is equal to either $\mathbf{e}_{1}$ or $\mathbf{e}_{2}$ for every $1 \leq j<p$.

We say a vector $\mathbf{x}=\left(x_{1}, \ldots, x_{k}\right) \in \mathbb{Z}^{2 \times k}$ is a $k$-point if $x_{1}, \ldots, x_{k}$ are distinct points in $\mathbb{Z}^{2}$. Suppose $\mathbf{x}=\left(x_{1}, \ldots, x_{k}\right)$ and $\mathbf{y}=\left(y_{1}, \ldots, y_{k}\right)$ are $k$-points and for each $i, \pi_{i}$ is a path from $x_{i}$ to $y_{i}$. If the sets $\pi_{1}, \ldots, \pi_{k}$ are disjoint, we say the $k$-tuple $\pi=\left(\pi_{1}, \ldots, \pi_{k}\right)$ is non-intersecting and refer to $\pi$ as a $k$-path. We write $\Gamma_{\mathbf{x} \rightarrow \mathbf{y}}$ for the set of $k$-paths from $\mathbf{x}$ to $\mathbf{y}$, and write $\mathbf{x} \leq \mathbf{y}$ whenever $\Gamma_{\mathbf{x} \rightarrow \mathbf{y}}$ is non-empty. (We emphasise that a $k$-path will always refer to a non-intersecting $k$-tuple of paths travelling north-east in $\mathbb{Z}^{2}$.)

Suppose further we have a random environment $\left\{\omega(z): z \in \mathbb{Z}^{2}\right\}$-a collection of independent and identically distributed finite-expectation random variables under a probability measure $\mathbb{P}$. For a $k$-path $\pi=\left(\pi_{1}, \ldots, \pi_{k}\right)$ from $\mathbf{x}$ to $\mathbf{y}$, we define the energy $F(\pi)$ of $\pi$ to be the sum of the weights included in the $k$-path, not including the starting points. That is, $F(\pi)$ is the random variable

$$
F(\pi):=\sum_{i=1}^{k} \sum_{z \in \pi_{i}-\left\{x_{i}\right\}} \omega(z) .
$$

Suppose $\beta$ is a real number such that $G(\beta):=\mathbb{E}\left[e^{\beta \omega(0,0)}\right]<\infty$. The goal of the present paper is to study the partition function

$$
Z_{\mathbf{x} \rightarrow \mathbf{y}}(\beta):=\sum_{\pi \in \Gamma_{\mathbf{x} \rightarrow \mathbf{y}}} \exp (\beta F(\pi))
$$

associated with the non-intersecting directed random polymer running from $\mathbf{x}$ to $\mathbf{y}$, with a particular emphasis on the asymptotics of $Z_{\mathbf{x} \rightarrow \mathbf{y}}(\beta)$ as the lengths of the paths and/or the number of paths grows to infinity. We emphasise that the assumptions $v:=\mathbb{E}[\omega(0,0)]<\infty$ and $G(\beta)=\mathbb{E}\left[e^{\beta \omega(0,0)}\right]<\infty$ are in force throughout the paper.

This article is divided into three interconnected parts. The first part is dedicated to proving asymptotic results for the partition functions in a general environment. In the second part we introduce a collection of random functions on the $N \times N$ square known as stochastic interfaces, and express the non-intersecting partition functions associated with a particular random environment in terms of a stochastic interface, developing connections with random matrix theory in the process. In the final part, we study the scaling limits of these interfaces as $N \rightarrow \infty$, relating our model to the Marčenko-Pastur law and Gaussian fields, and developing the machinery along the way to obtain new derivation of the surface tension of the bead model. In the following we overview each of these three respective parts in more detail. 
Before proceeding, we mention a couple of conventions we use to lighten notation. For real numbers $u$, let $\lfloor u\rfloor$ denote the largest integer less than or equal to $u$, and let $\lceil u\rceil$ denote the smallest integer greater than or equal to $u$. Whenever $\left(a_{n}\right)_{n \in \mathbb{Z}}$ are variables indexed by the integers, for real numbers $r$ we set $a_{r}:=a_{\lfloor r\rfloor}$, where $\lfloor r\rfloor$ is the largest integer not greater than $r$. We will use similar conventions throughout, in particular

whenever $c$ is positive and $N$ is an integer, $(N, c N)$ refers to the point $(N,\lfloor c N\rfloor)$ of $\mathbb{Z}^{2}$.

If $f$ is a function defined on a subset of $\mathbb{Z}^{2}$ or $\mathbb{R}^{2}$, we say $f$ is symmetric if $f(r, s)=f(s, r)$ for every $s$ and $r$. Finally, if $\mathbf{x}$ is a $k$-point and $a$ is an element of $\mathbb{Z}^{2}$, define the translation $\mathbf{x}+a$ to be the $k$-point whose $i^{\text {th }}$ component is given by $\mathbf{x}_{i}+a$.

\subsection{Non-intersecting Polymers}

The first part of this article is concerned with studying key properties of non-intersecting polymers in a general environment, with a particular focus on developing tools to study their asymptotics. These results are stated in full in Sect. 2, though we provide a brief outline here.

We say a $k$-point $\mathbf{x}=\left(x_{1}, \ldots, x_{k}\right)$ is nice if either $x_{i+1}$ lies strictly north and strictly west of $x_{i}$, or $x_{i+1}=x_{i}+\mathbf{e}_{2}$. This is a technical condition which roughly speaking ensures that $k$-paths going to and from a $k$-point $\mathbf{x}$ do not intersect. (See the beginning of Sect. 2 for a precise statement and proof.)

Our main tools for tackling the asymptotics of non-intersecting polymer partition functions $Z_{\mathbf{x} \rightarrow \mathbf{y}}(\beta)$ are the dual notions of series and parallel concatenation, which we now outline. Given a $k$-path $\pi$ from $\mathbf{x}$ to $\mathbf{y}$ and $k$-path $\gamma$ from $\mathbf{y}$ to $\mathbf{z}$, we may concatenate the paths $\pi$ and $\gamma$ in series to form a new $k$-tuple $\pi \oplus \gamma$ of paths from from $\mathbf{x}$ to $\mathbf{z}$. Provided the intermediate $k$-point $\mathbf{y}$ is nice, the concatenated path $\pi \oplus \gamma$ turns out to also be non-intersecting, and in this case the operation $\oplus$ is additive in the sense that

$$
F(\pi \oplus \gamma)=F(\pi)+F(\gamma),
$$

where $F$ is given as in (1.1). In particular, we have an $F$-preserving injection taking every pair of paths in $\Gamma_{\mathbf{x} \rightarrow \mathbf{y}} \times \Gamma_{\mathbf{y} \rightarrow \mathbf{z}}$ to a new path $\pi \oplus \gamma$ in $\Gamma_{\mathbf{x} \rightarrow \mathbf{z}}$. In Sect. 2, we use this injection to prove the series bound, which states that for nice $k$-points $\mathbf{x} \leq \mathbf{y} \leq \mathbf{z}$, the partition functions satisfy

$$
Z_{\mathbf{x} \rightarrow \mathbf{z}}(\beta) \geq Z_{\mathbf{x} \rightarrow \mathbf{y}}(\beta) Z_{\mathbf{y} \rightarrow \mathbf{z}}(\beta) .
$$

The series bound gives rise to subadditivity in the logarithmic partition functions $\log Z_{\mathbf{x} \rightarrow \mathbf{y}}(\beta)$, which we use in conjunction with Kingman's subadditive ergodic theorem [26] to obtain the logarithmic asymptotics of $Z_{\mathbf{x} \rightarrow \mathbf{y}}(\beta)$ as $\mathbf{x}$ and $\mathbf{y}$ grow far apart in the $(1, c)$ direction. Theorem 2.1 states that for any pair of nice $k$-points $\mathbf{x}$ and $\mathbf{y}$

$$
\frac{1}{N} \log Z_{\mathbf{x} \rightarrow \mathbf{y}+(N, c N)}(\beta) \text { converges to a deterministic limit } f_{c}(k, \beta),
$$

where the constant $f_{c}(k, \beta)$ is independent of $\mathbf{x}$ and $\mathbf{y}$. As we will see later, it is a natural consequence of the parallel bound that the limit $f_{c}(k, \beta)$ is concave in the $k$-variable, in that for any $j, k$ we have

$$
f_{c}(k+j, \beta) \leq f_{c}(k, \beta)+f_{c}(j, \beta) .
$$

However, it turns out that $f_{c}(k, \beta)$ has a linear dependence on $k$. Indeed, Theorem 2.2 states that 


$$
f_{c}(k, \beta)=k f_{c}(\beta),
$$

where we are writing $f_{c}(\beta)$ for $f_{c}(1, \beta)$.

Where series concatenation involves combining paths by glueing their ends together, parallel concatenation combines paths by letting them run side-by-side. Namely, any $(k+j)$ path $\pi$ has a unique decomposition $\pi=\pi^{\prime} \boxplus \pi^{\prime \prime}$ where $\pi^{\prime}$ is a $k$-path and $\pi^{\prime \prime}$ is a $j$-path. Again this procedure is additive in that $F\left(\pi^{\prime} \boxplus \pi^{\prime \prime}\right)=F\left(\pi^{\prime}\right)+F\left(\pi^{\prime \prime}\right)$, and in Sect. 2.1 we use it prove the parallel bound

$$
Z_{\mathbf{x} \rightarrow \mathbf{y}}(\beta) \leq Z_{\mathbf{x}^{\prime} \rightarrow \mathbf{y}^{\prime}}(\beta) Z_{\mathbf{x}^{\prime \prime} \rightarrow \mathbf{y}^{\prime \prime}}(\beta),
$$

where $\mathbf{x}:=\left(x_{1}, \ldots, x_{k+j}\right), \mathbf{x}^{\prime}=\left(x_{1}, \ldots, x_{k}\right)$ and $\mathbf{x}^{\prime \prime}=\left(x_{k+1}, \ldots, x_{k+j}\right)$, and $\mathbf{y}, \mathbf{y}^{\prime}$ and $\mathbf{y}^{\prime \prime}$ are defined similarly.

The parallel bound (1.6) gives a subadditivity in the $k$-variable which allows us to prove a result analogous to (1.5) concerning infinitely many paths of finite length running sideby-side. Thereafter, we study the infinite temperature $(\beta=0)$ case, where the LindströmGessel-Viennot formula may be used in conjunction with Szegö's powerful limit theorem to obtain explicit expressions for the large- $k$ logarithmic asymptotics of the partition functions in terms of a Laurent series with binomial coefficients.

Finally we look at the case of many long paths. By studying the large- $N$ asymptotics of a variant of Macmahon's formula [42], in Lemma 2.6 we obtain explicit logarithmic asymptotics for the partition functions associated with the infinite temperature case. We then use Jensen's inequality to relate the positive temperature partition functions with their infinite temperature counterparts (Lemma 2.7), leading us to Corollary 2.8, which establishes $N^{2}$ behaviour for the logarithmic partition function when both the number of paths as well as their lengths are of order $N$.

\subsection{Stochastic Interfaces and Whittaker Measures}

In the second part of this article-the results of which we state in full in Sect. 3-we introduce stochastic interfaces, random functions defined on the square $S_{N}:=\{1, \ldots, N\}^{2}$ of $\mathbb{Z}^{2}$. With the exception of Theorem 3.7 which is new, our work in this section is dedicated to reformulating results from Baryshnikov [3] and Corwin et al. [14] in the language of stochastic interface models.

For a convex interaction potential $V$, the stochastic interfaces we consider are essentially random functions $\phi^{N}: S_{N} \rightarrow \mathbb{R}$ with distributions proportional to

$$
\exp \left(-\sum_{\langle x, y\rangle} V(\phi(y)-\phi(x))-\mu \sum_{x \in D_{N}} \phi(x)\right),
$$

where $D_{N}:=\{(i, i): i=1, \ldots, N\}$ is the diagonal of $S_{N}$, and $\langle x, y\rangle$ is the set of edges in $S_{N}$ directed in the north or east direction. The central idea connecting directed polymers with stochastic interfaces is Proposition 3.2, a restatement of a result by Corwin et al. [14], which states that the non-intersecting partition functions associated with a certain random environment may be jointly expressed in terms of a stochastic interface.

More specifically, let $\tau_{\mu}^{N}(m, k)$ be the partition function associated with a $k$-paths in an $m \times N$ rectangle, where the variables in the random environment are log-gamma distributed in that each $e^{\beta \omega(z)}$ has the inverse gamma law

$$
I_{\mu}(d s)=\frac{s^{-\mu-1} e^{-1 / s}}{\Gamma(\mu)} \mathbb{1}_{s>0} d s
$$


of parameter $\mu>0$. We now define a random function $\varphi_{\mu}^{N}: S_{N} \rightarrow \mathbb{R}$ in terms of the joint partition functions $\tau_{\mu}^{N}(m, k)$ by setting

$$
\varphi_{\mu}^{N}(i, j):=\log \left(\frac{\tau_{\mu}^{N}(N-j+i, i)}{\tau_{\mu}^{N}(N-j+i, i-1)}\right)
$$

for $i \leq j$ (with a similar definition holding for $i \geq j$ ). Based on the main result in [14], we obtain the statement of Proposition 3.2:

$$
\varphi_{\mu}^{N} \text { is a stochastic interface with interaction potential } V(u)=\exp (u) .
$$

The small- $\mu$ and large- $\mu$ deformations of the stochastic interface $\varphi_{\mu}^{N}$ have respective connections with the eigenvalues ensembles of random matrix theory and the partition functions of deterministic non-intersecting directed polymers. In the former case, by appealing to ideas in Baryshnikov [3], we show that an eigenvalue process $\varphi_{\mathrm{LUE}}^{N}$ associated with the minors of a random matrix from the Laguerre Unitary Ensemble may be expressed in terms of a stochastic interface with the hard-core interaction potential $V(u):=\infty \mathbb{1}_{u>0}$. We use the results of [14] to obtain Proposition 3.5, which states that

$$
\mu \varphi_{\mu}^{N} \text { converges in distribution to } \varphi_{\mathrm{LUE}}^{N} \text { as } \mu \rightarrow 0 .
$$

As is mentioned in [14], by using a tropicalisation of the underlying polymer, one can use the statement (1.9) to recover a result by Johannson [22] relating non-intersecting last passage percolation with exponential weights to the eigenvalues of a Laguerre matrix.

We have contrasting behaviour as $\mu \rightarrow \infty$. Namely, if we define the tilted interface by $\theta_{\mu}^{N}(i, j):=\varphi_{\mu}^{N}(i, j)+(2 N+1-(j+i)) \log \mu$, then according to Theorem 3.7 we have

$$
\theta_{\mu}^{N} \text { converges almost surely to a deterministic function } \theta_{\min }^{N} \text { as } \mu \rightarrow \infty \text {. }
$$

Moreover, the deterministic limit $\theta_{\mathrm{min}}^{N}$ is the minimiser of a discrete variational problem on the square $S_{N}$, and has an explicit representation in terms of factorials.

\subsection{Scaling Limits for Stochastic Interfaces}

The final part of this article, which is discussed in full in Sect. 4, is concerned with using variational heuristics to study the large- $N$ asymptotics of stochastic interfaces. In the interest of maintaining a steady flow of ideas, in this part of the article we will be content to provide a plausibility argument based on physical heuristics in place of a rigorous mathematical proof.

Following the exposition in Funaki and Spohn [20], our first tool for studying the macroscopics of stochastic interfaces is that of surface tension-an asymptotic measure of the energy cost for an interface to lie a certain tilt. With this concept at hand, we conjecture that for certain choices of interaction potential, the macroscopic shapes of certain square interfaces may be given as solutions to variational problems on the unit square $S:=[0,1]^{2}$. In particular we anticipate that if $\bar{\varphi}_{\mu}^{N}: S \rightarrow \mathbb{R}$ is a rescaling of the interface associated with the partition functions of the non-intersecting log-gamma polymer-with both the square $S_{N}$ and the height of the interface both rescaled by $\frac{1}{N}$-then

$$
\bar{\varphi}_{\mu}^{N} \text { converges to a deterministic limit } \xi_{\mu}: S \rightarrow \mathbb{R} \text { as } N \rightarrow \infty \text {. }
$$

Moreover, if we let $\sigma^{\exp }$ be the surface tension associated with the exponential interaction potential $V(u)=\exp (u)$, then we expect that the deterministic limit $\xi_{\mu}$ is the the minimiser of 


$$
\mathcal{E}_{\mu}[v]:=\int_{S} \sigma^{\exp }(\nabla v(s, t)) d s d t+\mu \int_{0}^{1} v(s, s) d s,
$$

where the minimisation is taken over all functions $v: S \rightarrow \mathbb{R}$ satisfying $v(1,1)=0$. Finally, the limit shape is related to the asymptotic partition functions of the non-intersecting directed $\log$-gamma polymer through the following equation. Let $\tau_{\mu}^{N}(m, k)$ be the partition function associated with $k$ paths on an $N \times m$ rectangle. Then these arguments suggest that

$$
\lim _{N \rightarrow \infty} \frac{1}{N^{2}} \log \tau_{\mu}^{N}(c N, \alpha N):=\int_{0}^{\alpha} \xi_{\mu}(u, 1-c+u) d u .
$$

Having taken $N \rightarrow \infty$, we now consider the behaviour of $\xi_{\mu}$ as the parameter $\mu$ varies. On the one hand, by considering the relationship the interface $\xi_{\mu}^{N}$ has with the eigenvalues of Laguerre matrices, taking a large- $N$ analogue of (1.9) we anticipate that

$$
\mu \xi_{\mu} \text { converges to } \xi_{\mathrm{mp}} \text { as } \mu \rightarrow 0,
$$

where $\xi_{\mathrm{mp}}$ has an explicit expression in terms of the Marčenko-Pastur distribution. On the other hand, in the large- $\mu$ case we sketch an argument suggesting that

$$
\xi_{\mu}(s, t)+(2-s-t) \log \mu \text { converges to } \xi_{\mathrm{ht}}(s, t) \text { as } \mu \rightarrow \infty,
$$

where $\xi_{\mathrm{ht}}(s, t)$ has an explicit expression in terms of the function $q(u)=u \log u$. Given their own direct representations as scaling limits of stochastic interfaces, we also expect both limit functions $\xi_{\mathrm{mp}}$ and $\xi_{\mathrm{ht}}$ to be solutions of explicit variational problems.

Thereafter, we take a more refined argument to study the high-temperature limit—using the central limit theorem in place of the law of large numbers-with $\mu$ and $N$ sent to $\infty$ together through the scaling limit $\mu=\kappa N^{2}$. Indeed, Conjecture 4.7 states that the random processes $H_{\kappa}^{N}:=\left\{H_{\kappa}^{N}(s, t): 0 \leq s \leq t \leq 1\right\}$ given by

$$
H_{\kappa}^{N}(s, t):=k(N+m-k)(\log \kappa+2 \log N)+\log \tau_{\kappa N^{2}}^{N}(m, k)-\log \# \Gamma^{N}(m, k)
$$

converge in distribution to a centred Gaussian process, and gives a prediction for the covariance functions in terms of the asymptotic densities of a path model.

Finally, having developed the surface tension framework to study the macroscopics of stochastic interfaces, we use a version of the classical semicircle law of random matrix theory to reverse-engineer a straightforward derivation of a formula for the surface tension associated with the bead model [4]. To our knowledge, the only other place this formula appears in the literature is in the work of Sun [43], who provides a sophisticated derivation involving scaling limits of dimer models [9].

\subsection{Outline of the Paper}

The remainder of the paper is structured as follows. In Sect. 2, we discuss the asymptotics of non-intersecting directed polymers in random environments with a general weight distribution, giving full statements of the results overviewed in Sect. 1.2. In Sect. 3, we introduce stochastic interfaces and their connections with random matrices, fleshing out the description seen in Sect. 1.3. Finally, in Sect. 4, we study the large- $N$ asymptotics of the interfaces seen in Sect. 3, expanding on the discussion in Sect. 1.4.

The following sections of the paper, Sects. 5 through 8 , are dedicated to proving results stated in Sects. 2 through 4 , and at times providing further details for computations made in these earlier sections. In particular, Sects. 5 and 6 are dedicated to proving the results stated in Sects. 2 and 3 respectively. The proofs and further details surrounding the discussion in Sect. 
4 are spread across two sections, the latter of which, Sect. 8, is dedicated to our derivation of the surface tension of the bead model.

\section{Non-intersecting Polymers}

\subsection{Series and Parallel Inequalities}

Recall the series bound (1.3) and parallel bound (1.6) first introduced in Sect. 1.2. We now take a moment to give proofs of these two inequalities.

First we consider the series bound (1.3), which we prove using concatenation in series as follows. Suppose $x, y, z$ are points in $\mathbb{Z}^{2}$, and $\pi=\left\{a_{0}, \ldots, a_{p}\right\}$ is a 1-path from $x$ to $y$, and $\gamma=\left\{b_{0}, \ldots, b_{q}\right\}$ is a 1-path from $y$ to $z$. Then we define the concatenated 1-path $\pi \oplus \gamma:=\left\{c_{0}, \ldots, c_{p}, c_{p+1}, \ldots, c_{p+q}\right\}$ from $x$ to $z$ by setting $c_{i}=a_{i}$ for $i \leq p$ and $c_{i}:=b_{i-p}$ for $i>p$. It is plain to check that this is indeed a path from $x$ to $z$, and that

$$
F(\pi \oplus \gamma)=F(\pi)+F(\gamma)
$$

Now suppose $\mathbf{x}, \mathbf{y}$ and $\mathbf{z}$ are $k$-points satisfying $\mathbf{x} \leq \mathbf{y} \leq \mathbf{z}$. If $\pi$ is a $k$-path from $\mathbf{x}$ to $\mathbf{y}$ and $\gamma$ is a $k$-path from $\mathbf{y}$ to $\mathbf{z}$, then we may form a $k$-tuple $\pi \oplus \gamma:=\left(\pi_{1} \oplus \gamma_{1}, \ldots, \pi_{k} \oplus \gamma_{k}\right)$ of paths from $\mathbf{x}$ to $\mathbf{z}$, which may or may not be intersecting. Recall that we say a $k$-point $\left(x_{1}, \ldots, x_{k}\right)$ is nice if either $x_{i+1}$ lies both strictly north and strictly west of $x_{i}$, or $x_{i+1}=x_{i}+\mathbf{e}_{2}$. We now prove the series bound (1.3), which hinges on the observation that whenever the intermediate point $\mathbf{y}$ is nice, then this resulting $k$-tuple $(\pi \oplus \gamma)$ is guaranteed to be non-intersecting.

Proof of the Series Bound (1.3) First we show that if $\mathbf{y}$ is nice, then $\pi \oplus \gamma$ is a $k$-path-that is the sets $\left(\pi_{i} \oplus \gamma_{i}\right)$ and $\left(\pi_{j} \oplus \gamma_{j}\right)$ are disjoint for each $i \neq j$. Since each of $\pi$ and $\gamma$ are themselves $k$-paths, it is sufficient to show that for each $i \neq j$, the sets $\pi_{i}$ and $\gamma_{j}$ are disjoint.

To see this, first consider the case $i<j$. In this case $y_{j}$ lies strictly north of $y_{i}$, and hence every point of $\gamma_{j}$ lies strictly to the north of every point of $\pi_{i}$, and hence $\pi_{i} \cap \gamma_{j}=\varnothing$. Alternatively, consider the case $i>j$. Since $y$ is nice, either $y_{i}$ lies both strictly north and strictly west of $y_{j}$, or $y_{i}$ is of the form $y_{i}=y_{j}+m \mathbf{e}_{2}$ for some positive integer $m$. In the former case, every point of $\gamma_{j}$ lies strictly east of every point of $\pi_{i}$, and hence $\pi_{i} \cap \gamma_{j}=\varnothing$. In the latter case, we must have $y_{j+1}=y_{j}+1 \mathbf{e}_{2}$, and in particular, $\gamma_{j}$ must go through $y_{j}$ and then $y_{j}+1 \mathbf{e}_{1}$ in order avoid $y_{j+1}=y_{j}+1 \mathbf{e}_{2}$. It follows that $\gamma_{j}-\left\{y_{j}\right\}$ consists of points that lie strictly east of $\pi_{i}$, and hence again $\pi_{i} \cap \gamma_{j}=\varnothing$.

We have proved that all cases $\pi_{i}$ and $\gamma_{j}$ are disjoint, and hence the $k$-tuple $\pi \oplus \gamma$ of paths from $\mathbf{x}$ to $\mathbf{z}$ is a $k$-path. In particular, $\oplus$ is an injection from $\Gamma_{\mathbf{x} \rightarrow \mathbf{y}} \times \Gamma_{\mathbf{y} \rightarrow \mathbf{z}} \rightarrow \Gamma_{\mathbf{x} \rightarrow \mathbf{z}}$. Observing that the $k$-paths $\pi$ and $\gamma$ only overlap at $\mathbf{y}$, we see that this operation is additive in the sense that

$$
F(\pi \oplus \gamma)=F(\pi)+F(\gamma)
$$

Now suppose we have three $k$-points $\mathbf{x} \leq \mathbf{y} \leq \mathbf{z}$ such that $\mathbf{y}$ is nice. Using the positivity of the summands in (1.2) with the fact that $\oplus$ is an injection to obtain the second line below, 
and (2.1) to obtain the third, we have

$$
\begin{aligned}
Z_{\mathbf{x} \rightarrow \mathbf{z}}(\beta) & :=\sum_{\theta \in \Gamma_{\mathbf{x} \rightarrow \mathbf{z}}} \exp (\beta F(\theta)) \\
& \geq \sum_{(\pi, \gamma) \in \Gamma_{\mathbf{x} \rightarrow \mathbf{y}} \times \Gamma_{\mathbf{y} \rightarrow \mathbf{z}}} \exp (\beta F(\pi \oplus \gamma)) \\
& =\sum_{\pi \in \Gamma_{\mathbf{x} \rightarrow \mathbf{y}}} \exp (\beta F(\pi)) \sum_{\gamma \in \Gamma_{\mathbf{y} \rightarrow \mathbf{z}}} \exp (\beta F(\gamma)) \\
& =: Z_{\mathbf{x} \rightarrow \mathbf{y}}(\beta) Z_{\mathbf{y} \rightarrow \mathbf{z}}(\beta),
\end{aligned}
$$

establishing the series bound (1.3).

We now consider the dual procedure of concatenation in parallel, giving a proof of the parallel bound (1.6).

Proof of the Parallel Bound (1.6) Suppose we have $(k+j)$-points $\mathbf{x}=\left(x_{1}, \ldots, x_{k+j}\right)$ and $\mathbf{y}=\left(y_{1}, \ldots, y_{k+j}\right)$. Then every non-intersecting $(k+j)$-path $\pi$ gives rise to a unique decomposition

$$
\pi=\pi^{\prime} \boxplus \pi^{\prime \prime},
$$

where $\pi^{\prime}$ is a non-intersecting $k$-path from $\mathbf{x}^{\prime}=\left(x_{1}, \ldots, x_{k}\right)$ to $\mathbf{y}^{\prime}=\left(y_{1}, \ldots, y_{k}\right)$, and $\pi^{\prime \prime}$ is a non-intersecting $j$-path from $\mathbf{x}^{\prime \prime}=\left(x_{k+1}, \ldots, x_{k+j}\right)$ to $\mathbf{y}^{\prime \prime}=\left(y_{k+1}, \ldots, y_{k+j}\right)$. This procedure gives us an injection from $\Gamma_{\mathbf{x} \rightarrow \mathbf{y}}$ to $\Gamma_{\mathbf{x}^{\prime} \rightarrow \mathbf{y}^{\prime}} \times \Gamma_{\mathbf{x}^{\prime \prime} \rightarrow \mathbf{y}^{\prime \prime}}$, and this injection is additive in the sense that

$$
F\left(\pi^{\prime} \boxplus \pi^{\prime \prime}\right)=F\left(\pi^{\prime}\right)+F\left(\pi^{\prime \prime}\right) .
$$

Using the positivity of the summands in (1.2) with the fact that the map $\pi \mapsto \pi^{\prime} \boxplus \pi^{\prime \prime}$ is an injection satisfying (2.2), we have

$$
\begin{aligned}
Z_{\mathbf{x} \rightarrow \mathbf{y}}(\beta) & :=\sum_{\pi \in \Gamma_{\mathbf{x} \rightarrow \mathbf{y}}} \exp (\beta F(\pi)) \\
& \leq \sum_{\pi^{\prime} \in \Gamma_{\mathbf{x}^{\prime} \rightarrow \mathbf{y}^{\prime}}} \exp \left(\beta F\left(\pi^{\prime}\right)\right) \sum_{\pi^{\prime \prime} \in \Gamma_{\mathbf{x}^{\prime \prime} \rightarrow \mathbf{y}^{\prime \prime}}} \exp \left(\beta F\left(\pi^{\prime \prime}\right)\right) \\
& =: Z_{\mathbf{x} \rightarrow \mathbf{y}}(\beta) Z_{\mathbf{y} \rightarrow \mathbf{z}}(\beta),
\end{aligned}
$$

proving (1.6).

We remark that by iterating (1.6), for any pair of $k$-points $\mathbf{x}=\left(x_{1}, \ldots, x_{k}\right)$ and $\mathbf{y}=$ $\left(y_{1}, \ldots, y_{k}\right)$ we obtain

$$
Z_{\mathbf{x} \rightarrow \mathbf{y}}(\beta) \leq \prod_{i=1}^{k} Z_{x_{i} \rightarrow y_{i}}(\beta) .
$$

In fact, thanks to the celebrated Lindström-Gessel-Viennot lemma [42], the $k$-path partition functions have a determinantal expression in terms of the one-point partition functions:

$$
Z_{\mathbf{x} \rightarrow \mathbf{y}}(\beta)=\operatorname{det}_{i, j=1}^{k}\left(Z_{x_{i} \rightarrow y_{j}}(\beta)\right) .
$$

In light of (2.4), the inequality (2.3) may be understood as an analogue of Hadamard's inequality for the random matrix $\left(Z_{x_{i} \rightarrow y_{j}}(\beta)\right)_{i, j=1}^{k}$. 


\subsection{The Single Path Partition Function}

The case $k=1$ corresponds to studying the asymptotics of the partition function $Z_{(1,1) \rightarrow(n, m)}(\beta)$ as $m$ and $n$ become large, and is widely discussed in the literature. By a standard subadditivity argument using the special case $k=1$ of the series bound (1.3) and Kingman's ergodic theorem [26], it can be shown that the almost-sure limit

$$
f_{c}(\beta):=\lim _{N \uparrow \infty} \frac{1}{N} \log Z_{(1,1) \rightarrow(N, c N)}(\beta)
$$

exists and is equal to $\sup _{N \geq 1} \frac{1}{N} \mathbb{E}\left[\log Z_{(1,1) \rightarrow(N, c N)}(\beta)\right]$. Though there are useful bounds for $f_{c}(\beta)$ (see for instance Comets [10]), explicit expressions for the its value remaining unknown but for a few cases which we now discuss.

First we consider the infinite temperature limit case $\beta=0$. In this case the partition function $Z_{(1,1) \rightarrow(n, m)}(0)$ is deterministic for every $(n, m)$, simply counting the number of paths starting at $(1,1)$ and ending at $(n, m)$. A straightforward computation using Stirling's formula tells us that the free energy $f_{c}(0)$ is given by

$$
f_{c}(0)=\lim _{N \rightarrow \infty} \frac{1}{N} \log \left(\begin{array}{c}
(c+1) N \\
N
\end{array}\right)=(c+1) \log (c+1)-c \log c .
$$

We are also able to make sense of the zero temperature limit - the asymptotic case where $\beta \rightarrow \infty$. Here, the partition function concentrates on the path maximising the energy $F(\pi)$. Namely, for any pair of points $x \leq y$ in $\mathbb{Z}^{2}$, we have

$$
\lim _{\beta \uparrow \infty} \frac{1}{\beta} \log Z_{x \rightarrow y}(\beta)=\max _{\pi \in \Gamma_{x \rightarrow y}} F(\pi) .
$$

In particular, without too much concern at this stage for the technical details surrounding the interchange of limits, we have

$$
\lim _{\beta \uparrow \infty} \frac{1}{\beta} f_{c}(\beta)=\lim _{N \uparrow \infty} \frac{1}{N} \max _{\pi \in \Gamma_{(1,1) \rightarrow(N, c N)}} F(\pi)=: \ell_{c}
$$

The value of $\ell_{c}$ is known in a few special cases. For example, in the case that each $\omega(z)$ is exponentially distributed with mean 1 Rost [39] showed that

$$
\ell_{c}=(1+\sqrt{c})^{2} .
$$

See [33] for further discussion of the single-path zero-temperature limit $\ell_{c}$.

Finally, there is one particular distribution for the random environment for which an explicit expression is known for $f_{c}(\beta)$ at a positive and finite value of $\beta$. Namely, in the case where each $e^{\beta \omega(z)}$ has the inverse-gamma distribution with parameter $\mu$ as in (1.8), Seppäläinen [40] discovered a remarkable underlying algebraic structure based around the beta-gamma algebra making the partition function exactly solvable. (We refer the reader to [10, Chap. 7] for an overview.) Indeed, according to [40, Theorem 2.4],

$$
f_{c}(\beta)=-\sup _{\theta \in[0, \mu]}\left(c \psi_{0}(\theta)+\psi_{0}(\mu-\theta)\right),
$$

where $\psi_{0}(\theta):=\Gamma^{\prime}(\theta) / \Gamma(\theta)$ is the digamma function. Let us also mention here the papers [2, $15,21,32,34-36]$ where similar formulae have been obtained for some other exactly solvable polymer models. 


\subsection{Asymptotics for Finitely Many Long Paths}

For nice $k$-points $\mathbf{x}$ and $\mathbf{y}$, we study the asymptotics of the partition function $Z_{\mathbf{x} \rightarrow \mathbf{y}+(N, c N)}(\beta)$ associated with the $k$-points stretched far apart in the $(1, c)$ asymptotic choice of direction. Here we are able to exploit the subadditivity due to the series bound (1.3) to prove the following results.

Theorem 2.1 There exists a function $f_{c}(k, \beta)$ such that for any pair of nice $k$-points $\mathbf{x}$ and $\mathbf{y}$, we have the almost sure convergence

$$
\frac{1}{N} \log Z_{\mathbf{x} \rightarrow \mathbf{y}+(N, c N)}(\beta) \rightarrow f_{c}(k, \beta) .
$$

The limit satisfies $f_{c}(k, \beta)=\sup _{N \geq 1} \mathbb{E}\left[\frac{1}{N} \log Z_{\mathbf{x} \rightarrow \mathbf{y}+(N, c N)}(\beta)\right]=\lim _{N \rightarrow \infty} \frac{1}{N} \mathbb{E}$ $\left[\log Z_{\mathbf{x} \rightarrow \mathbf{y}+(N, c N)}\right]$, and this quantity is independent of the choices $\mathbf{x}$ and $\mathbf{y}$.

We point out that by (2.5) and Theorem 2.1, by definition we have $f_{c}(1, \beta)=f_{c}(\beta)$. We also remark that by the parallel bound (1.6), it can be seen that the limits $f_{c}(k, \beta)$ satisfy the inequalities

$$
f_{c}(k+j, \beta) \leq f_{c}(k, \beta)+f_{c}(j, \beta),
$$

for positive integers $k$ and $j$. We can say something much stronger however. According to the following theorem, $f_{c}(k, \beta)$ grows linearly in $k$.

Theorem 2.2 The limits satisfy

$$
f_{c}(k, \beta)=k f_{c}(\beta) .
$$

Finally, we will also prove the following result allowing comparison of $f_{c}(\beta)$ for different values of $c$.

Theorem 2.3 Let $0<c<c^{\prime}$ be positive reals. Then we have

$$
f_{c}(\beta)+\left(c^{\prime}-c\right) \beta v \leq f_{c^{\prime}}(\beta) \leq \frac{c^{\prime}}{c} f_{c}(\beta)-\left(\frac{c^{\prime}}{c}-1\right) \beta v .
$$

Theorems 2.1, 2.2 and 2.3 are proved in Sect. 5.

In the next section we look at the case where there are many paths of finite length.

\subsection{Asymptotics for Many Paths of Finite Length}

Where in the last section we had finitely many paths and let their lengths tend to infinity, in this section we do the opposite, considering many non-intersecting paths of fixed length running side-by-side, and letting the number of paths tend to infinity. In this direction, for $x \in \mathbb{Z}^{2}$ and $h=\left(h_{1}, h_{2}\right) \in \mathbb{Z}^{2}$, define the stacked $k$-point $\mathbf{x}^{h \uparrow k}$ at $x$ in the $h$ direction by

$$
\mathbf{x}_{i}^{h \uparrow k}:=x+(i-1) h, \quad i=1, \ldots, k .
$$

For $x \leq y$ in $\mathbb{Z}^{2}$, and a direction $h$ such that $h_{1} \leq 0<h_{2}$, we now consider the the large $k$-asymptotics of the random variable $Z_{\mathbf{x}^{h \uparrow k} \rightarrow \mathbf{y}^{h \uparrow k}}(\beta)$. The parallel bound (1.6) gives us a subadditivity in the $k$-variable which we use to prove the following result. 
Theorem 2.4 Let $x \leq y$ be points in $\mathbb{Z}^{2}$, and $h=\left(h_{1}, h_{2}\right)$ such that $h_{1} \leq 0<h_{2}$. Then as $k \rightarrow \infty$, the random variable $\frac{1}{k} \log Z_{\mathbf{x}^{h \uparrow k} \rightarrow \mathbf{y}^{h \uparrow k}}(\beta)$ converges almost surely to a deterministic limit $I_{y-x, h}(\beta)$.

In fact, in the infinite temperature case $(\beta=0)$, for certain choices of $h$, the asymptotic limit $I_{z, h}(0)$ can be computed explicitly by using the asymptotic theory of Toeplitz determinants [5]. In this direction we define the symbol associated with $z$ and $h$ to be the Laurent series $a_{z, h}: \mathbb{T} \rightarrow \mathbb{C}$ on the unit circle given by

$$
a_{z, h}(s):=\sum_{m \in \mathbb{Z}} Z_{0 \rightarrow z+m h}(0) s^{m} .
$$

Every continuous function $a: \mathbb{T} \rightarrow \mathbb{C}$ has a unique decomposition $a\left(e^{i t}\right)=\left|a\left(e^{i t}\right)\right| e^{i c(t)}$ where $c:[0,2 \pi) \rightarrow \mathbb{R}$ is a continuous function satisfying $c(0)=0$. The winding number of $a$ is the integer

$$
\operatorname{wind}(a):=\frac{1}{2 \pi} \lim _{t \rightarrow 2 \pi} c(t) .
$$

Using Szegö's limit theorem for the asymptotics of Toeplitz determinants, in Sect. 5.6 we prove the following result, which gives an expression for $I_{z, h}(0)$ in terms of the symbol $a_{z, h}$.

Theorem 2.5 Suppose that $h_{1}<0<h_{2}$ and the winding number of the symbol $a_{z, h}$ is zero. Then there is a Laurent series $\sum_{m \in \mathbb{Z}} c_{m} s^{m}$ satisfying

$$
a_{z, h}(s)=\exp \left(\sum_{m \in \mathbb{Z}} c_{m} s^{m}\right)
$$

such that

$$
\lim _{k \rightarrow \infty}\left(e^{-k c_{0}} Z_{\mathbf{x}^{h \uparrow k} \rightarrow \mathbf{y}^{h \uparrow k}}(0)\right)=\exp \left(\sum_{m=1}^{\infty} m c_{m} c_{-m}\right) .
$$

In particular, $I_{z, h}(0)=c_{0}$.

To see an example of this result in action, let $y=(3,2), x=(0,0)$, and let $h=(-2,2)$. To first get a rough idea of the asymptotics using the iterated parallel bound (2.3), we note that there are $\left(\begin{array}{c}3+2 \\ 2\end{array}\right)=10$ paths from $x$ to $y$, from which it follows that $Z_{0^{h \uparrow k} \rightarrow z^{h \uparrow k}}(0) \leq 10^{k}$, and hence $I_{(3,2),(-2,2)}(0) \leq \log 10$.

We now obtain the exact asymptotics using Theorem 2.5. First we note that the symbol associated with $x, y$ and $h$ is given by

$$
a_{y-x, h}(s):=\sum_{m \in \mathbb{Z}}\left(\begin{array}{c}
5 \\
3+2 m
\end{array}\right) s^{m}=\frac{5}{s}+10+s .
$$

We remark that $\operatorname{Re}\left(a_{y-x, h}\left(e^{i t}\right)\right)=6 \cos (t)+10$ for each $t$ in $[0,2 \pi)$. It follows that $a_{y-x, h}$ has positive real part on $\mathbb{T}$, and hence has winding number 0 . It is straightforward to show that the symbol has representation

$$
a_{y-x, h}(s)=\exp \left(\log (5+2 \sqrt{5})+\sum_{m=1}^{\infty} \frac{(-1)^{m}}{m}\left(\frac{c}{10} s\right)^{m}+\sum_{m=1}^{\infty} \frac{(-1)^{m}}{m}\left(\frac{c}{2} s^{-1}\right)^{m}\right) .
$$

It then follows from Theorem 2.5 that

$$
I_{(3,2),(-2,2)}=\log (5+2 \sqrt{5}) \approx \log 9.472 \leq \log 10 .
$$




\subsection{Asympotics for Many Long Paths}

Finally, in this section we consider the asymptotics of the partition functions associated with many paths in a rectangle whose dimensions are growing to infinity together with the number of paths. For $k \geq 1$ and $x \in \mathbb{Z}^{2}$, we define the stacked $k$-point $\mathbf{x}^{\uparrow k}$ above and below a point $x$ by

$$
\mathbf{x}_{i}^{\uparrow k}:=x+(i-1) \mathbf{e}_{2}, \quad \mathbf{x}_{i}^{\downarrow k}:=x+(i-k) \mathbf{e}_{2}, \quad i=1, \ldots, k .
$$

For integers $m, n, k$, consider $k$-points $(1,1)^{\uparrow k}$ and $(n, m)^{\downarrow k}$ at the bottom-left and top-right corner of an $m \times n$ rectangle. Then there is at least one $k$-path from $(1,1)^{\uparrow k}$ to $(n, m)^{\downarrow k}$ if and only if $k \leq m \wedge n$. With this picture in mind, we consider the scaling regime $m=c N, n=$ $N, k=\alpha N$ for $0<\alpha \leq c \leq 1$, leading us to study the asymptotic growth of the random variable

$$
\frac{1}{N^{2}} \log Z_{(1,1) \uparrow \alpha N} \rightarrow(N, c N)^{\downarrow \alpha N}(\beta) .
$$

To understand the $\frac{1}{N^{2}}$ scaling, it is useful first to consider the infinite-temperature limit $\beta=0$, which amounts to a computation calculating the asymptotic size of the set $\left.\Gamma_{(1,1)}\right)^{\uparrow k} \rightarrow(n, m) \downarrow k$ of $k$-paths on the $m \times n$ rectangle. Indeed, in Section 5.7, we exploit determinantal identities to prove the following result.

Lemma 2.6 As $N \rightarrow \infty, \frac{1}{N^{2}} \log Z_{(1,1)^{\uparrow \alpha N} \rightarrow(N, c N)^{\downarrow \alpha N}}(0) \rightarrow w(c, \alpha)$, where the limit $w(c, \alpha)$ is given by

$$
w(c, \alpha)=Q(1+c-\alpha)+Q(1-\alpha)+Q(c-\alpha)+Q(\alpha)-Q(c)-Q(c+1-2 \alpha),
$$

and $Q(u)=\frac{u^{2}}{2} \log u$.

With the scaled- $k$ high temperature limit at hand, in Section 5.1 we use Jensen's inequality to prove the following result relating the positive temperature partition function to the infinitetemperature limit. For points $a=\left(a^{1}, a^{2}\right)$ and $b=\left(b^{1}, b^{2}\right)$ of $\mathbb{Z}^{2}$, define $\|a-b\|_{1}:=$ $\left|a^{1}-b^{1}\right|+\left|a^{2}-b^{2}\right|$.

Lemma 2.7 Let $\mathbf{x}$ and $\mathbf{y}$ be $k$-points such that $\mathbf{x} \leq \mathbf{y}$, and let $\varpi(\mathbf{x}, \mathbf{y}):=\sum_{i=1}^{k}\left\|y_{i}-x_{i}\right\|_{1}$ be the number of environment weights in a $k$-path from $\mathbf{x} \rightarrow \mathbf{y}$ (not including the starting points). Suppose that $v:=\mathbb{E}[\omega(0,0)]$ and $G(\beta):=\mathbb{E}\left[e^{\beta \omega(0,0)}\right]$ are finite. Then

$$
\beta v \leq \frac{1}{\varpi(\mathbf{x}, \mathbf{y})} \mathbb{E} \ln \frac{Z_{\mathbf{x} \rightarrow \mathbf{y}}(\beta)}{Z_{\mathbf{x} \rightarrow \mathbf{y}}(0)} \leq \log G(\beta) .
$$

Now define the upper and lower limits

$$
\begin{aligned}
& R^{-}(c, \alpha ; \beta):=\liminf _{N \rightarrow \infty} \frac{1}{N^{2}} \mathbb{E} \log Z_{(1,1)^{\uparrow \alpha N} \rightarrow(N, c N)^{\downarrow \alpha N}}(\beta), \\
& R^{+}(c, \alpha ; \beta):=\limsup _{N \rightarrow \infty} \frac{1}{N^{2}} \mathbb{E} \log Z_{(1,1)^{\uparrow \alpha N} \rightarrow(N, c N)^{\downarrow \alpha N}}(\beta) .
\end{aligned}
$$

The following result is an immediately corollary of Lemma 2.6 and Lemma 2.7, showing that these lower and upper limits may be sandwiched within terms involving the infinite temperature limit. 
Corollary 2.8 The asymptotic $k$-path partition functions satisfy

$$
\begin{aligned}
\alpha(1+c-\alpha) \beta v & \leq R^{-}(c, \alpha ; \beta)-w(c, \alpha) \leq R^{+}(c, \alpha ; \beta)-w(c, \alpha) \\
& \leq \alpha(1+c-\alpha) \log G(\beta),
\end{aligned}
$$

where $v=\mathbb{E}[\omega(0,0)], G(\beta)=\mathbb{E}\left[e^{\beta \omega(0,0)}\right]$, and $w(c, \alpha)$ is the many-path zero temperature limit defined in Lemma 2.6.

Let us remark that it is immediate from Lemma 2.6 that the upper and lower limits (2.15) and (2.16) agree in the special case $\beta=0$. Moreover, a computation involving shows that

$$
\left.\frac{d}{d \alpha} w(c, \alpha)\right|_{\alpha=0}=f_{c}(0)
$$

where $f_{c}(0)$ is given in (2.6). We now conjecture that this relationship holds more generally.

Conjecture 2.9 The lower and upper limits $R^{-}(c, \alpha, \beta)$ and $R^{+}(c, \alpha, \beta)$ agree, and moreover in this case

$$
\left.\frac{d}{d \alpha} R^{+}(c, \alpha ; \beta)\right|_{\alpha=0}=f_{c}(\beta)
$$

\section{Stochastic Interfaces}

\subsection{Stochastic Interfaces}

Let $\Lambda$ be a finite subset of $\mathbb{Z}^{2}$ and let $\Lambda^{*}:=\left\{\langle x, y\rangle \in \Lambda^{2}: y-x\right.$ is equal to $\mathbf{e}_{1}$ or $\left.\mathbf{e}_{2}\right\}$ be the set of directed edges in $\Lambda$. Suppose now $V$ is a convex function on $\mathbb{R}$ and $\left(W_{x}: x \in \Lambda\right)$ are weight functions. A stochastic interface is a random function $\phi: \Lambda \rightarrow \mathbb{R}$ whose law is given by $Z^{-1} \exp \left(-H_{\Lambda}[\phi]\right) \prod_{x \in \Lambda} d \phi(x)$, where $H_{\Lambda}$ is the Hamiltonian

$$
H_{\Lambda}[\phi]:=\sum_{\langle x, y\rangle \in \Lambda^{*}} V(\phi(y)-\phi(x))+\sum_{x \in \Lambda} W_{x}(\phi(x)),
$$

and $Z:=\int_{\mathbb{R}^{\Lambda}} \exp \left(-H_{\Lambda}[\phi]\right) \prod_{x \in \Lambda} d \phi(x)$ is the interface partition function. We observe that if $D$ is a subset of $\Lambda$, then the marginal law of $\phi$ on $D$ may be obtained by integrating out $\phi$ on $\Lambda-D$. Namely, for any function $\lambda: D \rightarrow \mathbb{R}$ defined on a subset $D$ of $\Lambda$,

$$
P(\phi(y) \in d \lambda(y), y \in D)=\frac{g_{D}(\lambda)}{Z} \prod_{y \in D} d \lambda(y),
$$

where $g_{D}: \mathbb{R}^{D} \rightarrow \mathbb{R}$ is the energy integral

$$
g_{D}(\lambda):=\int_{\mathbb{R}^{\Lambda}} \exp \left(-H_{\Lambda}[\phi]\right) \prod_{y \in D} \delta_{\lambda(y)}(d \phi(y)) \prod_{x \in \Lambda-D} d \phi(x) .
$$

We will be particularly interested in stochastic interfaces on triangular and square subsets of $\mathbb{Z}^{2}$. Let $T_{N}:=\{(i, j): 1 \leq i \leq j \leq N\}$ and $S_{N}:=\{(i, j): 1 \leq i, j \leq N\}$ be the triangle and the square in $\mathbb{Z}^{2}$, let $D_{N}:=\left\{(i, i) \in T_{N}\right\}$ be the set of diagonal entries and let $E_{N}:=T_{N}-D_{N}$ be the non-diagonal entries of the triangle. When $\Pi=D_{N}$, we call the 
energy integral a pattern integral and write $g:=g_{D_{N}}$ for short. In other words, a pattern integral is simply a function $g^{V}: \mathbb{R}^{N} \rightarrow \mathbb{R}$ given by

$$
g^{V}\left(\lambda_{1}, \ldots, \lambda_{N}\right):=\int_{\mathbb{R}^{T_{N}}} \exp \left(-H_{T_{N}}[\phi]\right) \prod_{x \in E_{N}} d \phi(x) \prod_{i=1}^{N} \delta_{\lambda_{i}}(d \phi(i, i)) .
$$

where $d \phi(x)$ is Lebesgue measure and $\delta_{u}$ is the Dirac mass at $u$.

Though the majority of surrounding literature on stochastic interfaces is restricted to models with symmetric interaction potentials, we will be most interested in the potentials associated with the exponential and bead interaction models, neither of which are symmetric. These are given by

$$
\exp (u):=e^{u} \text { and } \operatorname{bead}(u):=\infty \mathbb{1}_{u>0},
$$

respectively, and will appear in stochastic interfaces relating to random polymers and random matrices which we introduce in the following two sections.

For a moment let the weight functions $\left(W_{x}: x \in T_{N}\right)$ be zero and consider interfaces defined on the triangle $T_{N}$ with the bead interaction $V(u)=\operatorname{bead}(u)$. It is straightforward to see that the functions $\phi: T_{N} \rightarrow \mathbb{R}$ for which the Hamiltonian $H_{T_{N}}[\phi]$ is finite are precisely the Gelfand-Tsetlin patterns, namely those functions satisfying the inequalities

$$
\phi(i, j-1) \geq \phi(i, j) \geq \phi(i+1, j) \text { for every } 1 \leq i<j \leq N .
$$

Given a vector $\left(\lambda_{1}, \ldots, \lambda_{N}\right)$, we write $G T_{N}(\lambda)$ for the set of Gelfand-Tsetlin patterns $\phi$ : $T_{N} \rightarrow \mathbb{R}$ satisfying $(\phi(1,1), \ldots, \phi(N, N))=\left(\lambda_{1}, \ldots, \lambda_{N}\right)$. Clearly this set is empty unless $\lambda_{1} \geq \ldots \geq \lambda_{N}$. In fact, it is well-known that the pattern integral $g^{\text {bead }}$ associated with the $V(u)=\operatorname{bead}(u)$ is given by

$$
\begin{aligned}
g^{\text {bead }}(\lambda) & =\int_{\mathbb{R}^{T_{N}}} \prod_{\langle x, y\rangle \in T_{N}^{*}} \mathbb{1}_{\phi(y) \leq \phi(x)} \prod_{x \in E_{N}} d \phi(x) \prod_{i=1}^{N} \delta_{\lambda_{i}}(d \phi(i, i)) \\
& =\operatorname{Vol} G T_{N}(\lambda)=\frac{\Delta_{N}(\lambda)}{H(N)},
\end{aligned}
$$

where

$$
\Delta_{N}(\lambda):=\prod_{1 \leq i<j \leq N}\left(\lambda_{i}-\lambda_{j}\right) \mathbb{1}_{\lambda_{1} \geq \ldots \geq \lambda_{N}}
$$

and $H(N):=\prod_{j=0}^{N-1} j !$ is the superfactorial. By using the representation [29, Section I.3] of the product occuring in (3.4) as a determinant of a Vandermonde matrix:

$$
\prod_{1 \leq i<j \leq N}\left(\lambda_{i}-\lambda_{j}\right)=\operatorname{det}_{i, j=1}^{N}\left(\lambda_{i}^{N-j}\right),
$$

it is straightforward to prove the volume formula (3.3) by induction.

As for the exponential interaction potential exp, the associated pattern integral

$$
g^{\exp }(\lambda):=\int_{\mathbb{R}^{T_{N}}} \exp \left(-\sum_{(x, y) \in T_{N}^{*}} e^{\phi(y)-\phi(x)}\right) \prod_{x \in E_{N}} d \phi(x) \prod_{k=1}^{N} \delta_{\lambda_{k}}(d \phi(i, i))
$$

is known as a Whittaker function (with parameter 0 ). 
We record the following lemma, which we will use shortly to connect the diagonal entries of stochastic interfaces with both the eigenvalue ensembles of random matrix theory as well as the so-called Whittaker measure.

Lemma 3.1 Let $\phi^{N}: S_{N} \rightarrow \mathbb{R}$ be a stochastic interface with interaction potential $V$ such that the off-diagonal weight functions $\left(W_{x}: x \in E_{N}\right)$ are identically zero. Then the marginal law of the diagonal $\left(\lambda_{1}, \ldots, \lambda_{N}\right):=\left(\phi^{N}(1,1), \ldots, \phi^{N}(N, N)\right)$ is given by

$$
\frac{1}{Z} g^{V}(\lambda)^{2} \exp \left(-\sum_{i=1}^{N} W_{(i, i)}\left(\lambda_{i}\right)\right) \prod_{i=1}^{N} d \lambda_{i}
$$

Proof Integrating out the off-diagonal variables $(\phi(i, j))_{1 \leq i<j \leq N}$ and $(\phi(i, j))_{1 \leq j<i \leq N}$ and using the definition (3.1), we obtain two powers of $g^{V}(\lambda)$.

In the next section we discuss the Whittaker measure, a collection of random variables related to the partition functions of a random polymer which may be thought of in terms of a stochastic interface.

\subsection{The Whittaker Measure as a Stochastic Interface}

We will see now that for a random polymer with product weights distributed according to the inverse gamma law (1.8), the partition functions have an expression in terms of a stochastic interface. In this direction, first recall the inverse gamma distribution with parameter $\mu$

$$
I_{\mu}(d s)=\frac{s^{-\mu-1} e^{-1 / s}}{\Gamma(\mu)} \mathbb{1}_{s>0} d s,
$$

and let $F_{\mu}(s):=\int_{0}^{s} I_{\mu}(d u)$ be the associated distribution function. In order to consider the simultaneous behaviour of our partition functions as $\mu$ varies, we would like to take a coupling of our weight variables as functions of $\mu$. To this end, suppose $\left(U(z): z \in \mathbb{Z}^{2}\right)$ are uniformly distributed random variables on the unit interval, and for $z \in \mathbb{Z}^{2}$ define the random variable $\zeta_{\mu}(z):=F_{\mu}^{-1}(U(z))$. Clearly $\zeta_{\mu}(z)$ is inverse gamma distributed with parameter $\mu$.

For $z=(i, j)$, define $\tilde{z}:=(j, i)$. Now for $1 \leq k \leq m \leq N$, define the path set

$$
\Gamma^{N}(m, k):=\Gamma_{(1,1) \uparrow k} \rightarrow(N, m)^{\Downarrow k},
$$

and consider the associated partition functions

$$
\tau_{\mu}^{N}(m, k):=\sum_{\pi \in \Gamma^{N}(m, k)} \prod_{z \in \pi} \zeta_{\mu}(z), \quad \tilde{\tau}_{\mu}^{N}(m, k):=\sum_{\pi \in \Gamma^{N}(m, k)} \prod_{z \in \pi} \zeta_{\mu}(\tilde{z}) .
$$

We emphasise that in contrast to the partition functions studied in Sect. 2, the initial points $\{(1, i-1): i=1, \ldots, k\}$ of the $k$-paths $\pi$ in $\Gamma^{N}(m, k)$ or $\tilde{\Gamma}^{N}(m, k)$ are included in the weight products in (3.8). Now consider the random function $\varphi_{\mu}^{N}: S_{N} \rightarrow \mathbb{R}$ defined by

$$
\varphi_{\mu}^{N}(i, j)= \begin{cases}\log \left(\frac{\tau_{\mu}^{N}(N-j+i, i)}{\tau_{\mu}^{N}(N-j+i, i-1)}\right) & i \leq j . \\ \log \left(\frac{\tilde{\tau}_{\mu}^{N}(N-i+j, j)}{\tilde{\tau}_{\mu}^{N}(N-i+j, j-1)}\right) & i \geq j .\end{cases}
$$

with the conventions that $\tau_{\mu}^{N}(m, 0)=\tilde{\tau}_{\mu}^{N}(m, 0)=1$ for each $m$. By way of a diagram the reader may convince themselves that $\tau_{\mu}^{N}(N, k)=\tilde{\tau}_{\mu}^{N}(N, k)$ for every $k \leq N$, from which it 
follows that the overlapping definitions in (3.9) on the diagonal $i=j$ agree. We remark that (3.9) may be inverted to give

$$
\log \tau_{\mu}^{N}(m, k)=\sum_{i=1}^{k} \varphi_{\mu}^{N}(i, N-m+i)
$$

The following result is a consequence of results in [14] and [37], and is the key idea in this section, stating that $\varphi_{\mu}^{N}$ is a stochastic interface:

Proposition 3.2 The random function $\varphi_{\mu}^{N}: S_{N} \rightarrow \mathbb{R}$ given by (3.9) is a stochastic interface on $S_{N}$ with interaction potential $V(x)=\exp (x)$ and weight functions $W_{(i, j)}(u)=\mathbb{1}_{i=j} \mu u+$ $\mathbb{1}_{i=j=N} e^{-u}$. The interface partition function is given by $Z_{\mu}:=\Gamma(\mu)^{N^{2}}$.

In Sect. 6.1 we give an explanation for how Proposition 3.2 follows from [37]. We remark that using the definition $g^{\exp }$ in Lemma 3.1, it follows from Theorem 3.2 that the marginal law of the diagonal $\left(\lambda_{1}, \ldots, \lambda_{N}\right):=\left(\varphi_{\mu}^{N}(1,1), \ldots, \varphi_{\mu}^{N}(N, N)\right)$ may be given in terms of the so-called Whittaker measure with constant parameter $\mu$ :

$$
P_{\mu}^{N}(d \lambda):=\frac{1}{\Gamma(\mu)^{N^{2}}} \exp \left(-e^{-\lambda_{N}}-\mu \sum_{i=1}^{N} \lambda_{i}\right) g^{\exp }(\lambda)^{2} \prod_{i=1}^{N} d \lambda_{i} .
$$

We note that the interpretation of the log-gamma model as a stochastic interface has also been observed, in the language of Gibbsian line ensembles, by Wu [44]. Further related work on Gibbsian line ensembles and non-intersecting multi-path polymers can be found in the recent papers $[11-13,16]$.

\subsection{Eigenvalue Processes and $\mu \rightarrow 0$}

For an $N \times N$ matrix $A:=\left(A_{i, j}\right)_{1 \leq i, j \leq N}$, let $A^{(k)}:=\left(A_{i, j}\right)_{1 \leq i, j \leq k}$ be its principal $k \times k$ minor. Now suppose $H$ and $U$ are $N \times N$ complex matrices, where $H$ is Hermitian and $U$ is unitary. We define the eigenvalue process $\varphi_{H, U}: S_{N} \rightarrow \mathbb{R}$ associated with $H$ and $U$ to be the function $\varphi_{H, U}: S_{N} \rightarrow \mathbb{R}$ given by

$$
\varphi_{H, U}(i, j):=\left\{\begin{array}{l}
\text { The } i \text { th largest eigenvalue of } H^{(N-j+i)} \quad \text { if } i \leq j, \\
\text { The } j \text { th largest eigenvalue of }\left(U^{*} H U\right)^{(N-i+j)} \quad \text { if } i \geq j .
\end{array}\right.
$$

Let us remark that the $N \times N$ matrices $H$ and $U^{*} H U$ have the same eigenvalues, hence the two definitions in (3.12) are consistent on the diagonal $i=j$. We also point out that thanks to Cauchy's interlacing theorem, the eigenvalues of each $A^{(k)}$ interlace those of $A^{(k+1)}$. This tells us that for any directed edge $\langle x, y\rangle$ in $S_{N}^{*}, \varphi_{H, U}(x) \geq \varphi_{H, U}(y)$, or in other words, that $\varphi_{H, U}$ is a Gelfand-Tsetlin pattern on the subset $T_{N}$ of $S_{N}$.

We now consider the eigenvalue processes associated with certain ensembles of unitarily invariant random matrices, introducing the Gaussian Unitary Ensemble (GUE) and the Laguerre Unitary Ensemble (LUE). We say a random $N \times N$ complex Hermitian matrix is GUE distributed if its law is given by

$$
P_{\mathrm{GUE}}^{N}(d H):=A_{N} \exp \left(-\frac{1}{2} \operatorname{Tr}\left(H^{2}\right)\right) d H,
$$


and we say a random $M \times M$ complex Hermitian matrix is LUE distributed with underlying parameter $N$ if its law is given by

$$
P_{\text {LUE, } M}^{N}(d H):=B_{N, M} \operatorname{det}(H)^{N-M} \exp (-\operatorname{Tr}(H)) \mathbb{1}_{H \geq 0} d H,
$$

where $\mathbb{1}_{H \geq 0}$ is the indicator function on the set of positive definite matrices. When $M=N$ in (3.14), we just say $H$ is LUE distributed for short. Since both the trace and determinant are invariant under unitary conjugations, it follows that if $H$ has either of the laws (3.13) or (3.14), then for any unitary $U, U^{*} H U$ has the same law as $H$.

We will also use the following property of LUE matrices, which is straightforward to prove using the representation of Laguerre matrices as a product of matrices with independent complex Gaussian entries [18, Chap. 3].

Remark 3.3 If $H$ is distributed with law $P_{\mathrm{LUE}, N}^{N}$, then the marginal law of the minor $H^{(k)}$ is given by $P_{\mathrm{LUE}, k}^{N}$.

The following proposition, which states that the eigenvalue processes associated with GUE and LUE random matrices can be reformulated as stochastic interfaces, is a consequence of well known properties of the eigenvalues of unitarily invariant random matrices $[3,24]$.

Proposition 3.4 Suppose H is GUE distributed and U is Haar distributed on the set of unitary matrices. Then the eigenvalue process $\varphi_{H, U}$ associated with $(H, U)$ is a stochastic interface with interaction potential bead $(u)$ and weight functions

$$
W_{(i, j)}^{\mathrm{GUE}}(u):=\mathbb{1}_{i=j} \frac{1}{2} u^{2} .
$$

Alternatively, suppose $H$ is LUE distributed and $U$ is Haar distributed on the set of unitary matrices. Then the eigenvalue process $\varphi_{H, U}$ associated with $(H, U)$ is a stochastic interface with interaction potential bead $(u)$ and weight functions

$$
W_{(i, j)}^{\mathrm{LUE}}(u):=\mathbb{1}_{i=j} u+\mathbb{1}_{i=j=N} \infty \mathbb{1}_{u<0} .
$$

We provide a proof of Proposition 3.4 in Sect. 6.2. For short, we refer to the eigenvalue processes occuring in Proposition 3.4 as the GUE and LUE eigenvalue processes. With this result at hand (in fact for the moment we only require the part regarding LUE matrices), we are ready to establish the relationship between the partition functions of the polymer with inverse-gamma distributed weights and the eigenvalues of random matrices.

Theorem 3.5 Let $\varphi_{\mu}^{N}: S_{N} \rightarrow \mathbb{R}$ be the interface defined in (3.9). Then as $\mu \downarrow 0$, the rescaled process $\mu \varphi_{\mu}^{N}$ converges in distribution to the LUE eigenvalue process.

Sketch Proof By Theorem 3.2, $\varphi_{\mu}^{N}$ is a stochastic interface with the exponential interaction potential and weight functions $W_{(i, j)}(u)=\mathbb{1}_{i=j} \mu u+\mathbb{1}_{i=j=N} e^{-u}$. A calculation shows that the change of variable $\theta_{\mu}^{N}:=\mu \varphi_{\mu}^{N}$ is also a stochastic interface with interaction potential $V(u)=e^{\mu^{-1} u}$ and weight functions $W_{(i, j)}(u)=\mathbb{1}_{i=j} u+\mathbb{1}_{i=j=N} e^{-\mu^{-1} u}$, which converge as $\mu \downarrow 0$ to the interaction potential $V(u)=\infty \mathbb{1}_{u>0}$ and weight function $W_{(i, j)}^{\mathrm{LUE}}(u):=$ $\mathbb{1}_{i=j} u+\mathbb{1}_{i=j=N} \infty \mathbb{1}_{u<0}$ associated with the LUE eigenvalue process.

See Sect. 6.3 for a rigorous proof of Theorem 3.5 using the dominated convergence theorem.

We now consider the implications of this connection by looking directly at the small$\mu$ asymptotics of the polymer. Suppose $\zeta_{\mu}$ is inverse-gamma distributed with parameter 
$\mu$, and let $\chi_{\mu}:=\mu \log \zeta_{\mu}$. Using the small- $\mu$ asymptotics of the Gamma function, it is straightforward to verify that

$$
\mathbb{E}\left[e^{-s \chi_{\mu}}\right]=\frac{\Gamma(\mu(s+1))}{\Gamma(\mu)} \rightarrow \frac{1}{1+s} \text { as } \mu \downarrow 0 .
$$

That is, $\chi_{\mu}$ converges in distribution to a standard exponential random variable as $\mu \downarrow 0$. Applying these facts to the partition functions (3.8), we see that as $\mu \downarrow 0$ we have the convergence in distribution

$$
\begin{aligned}
\mu \log \tau_{\mu}^{N}(m, k)= & \mu \log \sum_{\pi \in \Gamma^{N}(m, k)} \exp \left(\frac{1}{\mu} \sum_{z \in \pi} \mu \log \zeta_{\mu}(z)\right) \\
& \rightarrow^{(d)} \max _{\pi \in \Gamma^{N}(m, k)} \sum_{z \in \pi} e(z)=: L^{N}(m, k),
\end{aligned}
$$

where $\left(e(z): z \in \mathbb{Z}^{2}\right)$ are independent standard exponential random variables.

On the other hand, as $\mu \downarrow 0$, combining (3.10) and Theorem 3.5, we have the convergence in distribution

$$
\mu \log \tau_{\mu}^{N}(m, k):=\sum_{i=1}^{k} \mu \varphi_{\mu}^{N}(i, N-m+i) \rightarrow \sum_{i=1}^{k} \varphi_{\mathrm{LUE}}^{N}(i, N-m+i) .
$$

Comparing (3.15) with (3.16) we obtain the distributional equality

$$
L^{N}(m, k)={ }^{(d)} \sum_{i=1}^{k} \varphi_{\mathrm{LUE}}^{N}(i, N-m+i) .
$$

By using the definition (3.12) in (3.17) and considering Remark (3.3), we recover the following result by Johansson [22, Proposition 1.4] connecting the eigenvalues of LUE matrices with non-intersecting last passage percolation on an exponential polymer. (See also Doumerc [17].)

Proposition 3.6 The random variable $L^{N}(m, k)$ is equal in law to the sum of the $k$ largest eigenvalues of an $m \times m$ Laguerre matrix with underlying parameter $N$.

\subsection{The $\mu \rightarrow \infty$ Limit}

Where in the last section we considered the small- $\mu$ asymptotics of the interface $\varphi_{\mu}^{N}$, here we consider the large- $\mu$ asymptotics. The following theorem states that as $\mu \rightarrow \infty$, a suitable rescaling of the $\varphi_{\mu}^{N}$ converges to a explicit deterministic shape related to a combinatorial problem.

Theorem 3.7 Let $\theta_{\mu}^{N}: S_{N} \rightarrow \mathbb{R}$ be the rescaled interface

$$
\theta_{\mu}^{N}(i, j):=\varphi_{\mu}^{N}(i, j)+(2 N+1-(j+i)) \log \mu .
$$

Then as $\mu \rightarrow \infty, \theta_{\mu}^{N}$ converges almost-surely to a deterministic function $\theta_{\min }^{N}: S_{N} \rightarrow \mathbb{R}$, where $\theta_{\min }$ is the symmetric function given by

$$
\theta_{\min }^{N}(i, j):=\log \left(\frac{(i-1) !(2 N-j-i+1) !(2 N-j-i) !}{(2 N-j) !(N-j) !(N-i) !}\right), \quad i \leq j .
$$


Moreover, the function $\theta_{\min }^{N}$ is the minimiser of the energy functional $\mathcal{F}: \mathbb{R}^{S_{N}} \rightarrow \mathbb{R}$ given by

$$
\mathcal{F}^{N}[\theta]:=e^{-\theta(N, N)}+\sum_{i=1}^{N} \theta(i, i)+\sum_{\langle x, y\rangle \in S_{N}^{*}} e^{\theta(y)-\theta(x)} .
$$

Theorem 3.7 is proved in Sect. 6.4 by directly analysing the large- $\mu$ behaviour of the interface $\varphi_{\mu}^{N}$, and studying the relationship this interface has with a deterministic polymer via the application of the law of large numbers to $1 / \zeta_{\mu}(z)$ (which for integer values of $\mu$ has a representation at the sum of $\mu$ independent exponential random variables). The fact that the function $\theta_{\min }^{N}$ minimises $\mathcal{F}^{N}[\theta]$ is an offshoot of our result, and we suspect there is a more direct combinatorial proof. That completes the section on finite interfaces. In the next section, we look at the asymptotics of these interfaces as $N \rightarrow \infty$.

\section{Scaling Limits of Stochastic Interfaces}

\subsection{Surface Tension and Asymptotics of Stochastic Interfaces}

In this section we will be interested in applying thermodynamic heuristics to study the macroscopic behaviour of the stochastic interfaces seen in the previous section, formulating their limiting shapes in terms of variational problems, and using these limit shapes to anticipate the asymptotics of the partition functions associated with the non-intersecting log-gamma polymer.

We begin by introducing surface tension, an asymptotic measure of the cost of an interface to lie at a certain tilt. For further information on surface tension, the reader is referred to Sheffield [41] and Funaki [19]. Let $\partial S_{N}$ and $S_{N}^{\circ}$ denote the boundary and the interior of the square $S_{N}$. Consider the square Hamiltonian

$$
H_{S_{N}}[\phi]:=\sum_{\langle x, y\rangle \in S_{N}^{*}} V(\phi(y)-\phi(x))
$$

associated with an interaction potential $V$ and $W_{x} \equiv 0$ for every $x$. For $N \geq 3$, we define the finite surface tension of interaction potential $V$ at tilt $(p, q) \in \mathbb{R}^{2}$ by

$\sigma_{N}^{V}(p, q):=-\frac{1}{(N-2)^{2}} \log \left(\int_{\mathbb{R}^{\Lambda_{N}}} \exp \left(-H_{S_{N}}[\phi]\right) \prod_{y \in \partial S_{N}} \delta_{\left(p y_{1}+q y_{2}\right)}(d \phi(x)) \prod_{x \in S_{N}^{\circ}} d \phi(x)\right)$.

The following result by Funaki and Spohn [20] states that under relatively strong conditions on the interaction potential $V$, the finite surface tensions $\sigma_{N}^{V}$ converge to a limit as a $N \rightarrow \infty$. The limit $\sigma^{V}$ is called the surface tension associated with $V$.

Proposition 4.1 Suppose the potential function $V$ is symmetric, twice differentiable, and satisfies $c_{-} \leq V^{\prime \prime}(x) \leq c_{+}$for some positive reals $c_{-} \leq c_{+}$. Then the limit $\sigma^{V}(p):=$ $\lim _{N \rightarrow \infty} \sigma_{N}^{V}(p)$ exists and is a convex function on $\mathbb{R}^{2}$.

Neither of the interaction potentials bead and exp are symmetric, nor do they satisfy the technical condition required by Funaki and Spohn for the existence of the associated surface 
tension. Nonetheless, it was established by Sun [43] that we have the existence of the surface tension

$$
\sigma^{\text {bead }}(p, q):=\lim _{N \rightarrow \infty} \sigma_{N}^{\text {bead }}(p, q)
$$

associated with the $\operatorname{bead}(u)$ interaction, and moreover, Sun provided a formula for $\sigma^{\text {bead }}(p, q)$ in a different coordinate system which we discuss below. Based on the relative similarity between the exponential and bead interaction, we anticipate the following conjecture.

Conjecture 4.2 The surface tension $\sigma^{\exp }:=\lim _{N \rightarrow \infty} \sigma_{N}^{\exp }$ associated with the exponential interaction exists.

With the definition of surface tension at hand, we are ready to study the macroscopics of stochastic interfaces. First we define a rescaling of stochastic interfaces from $S_{N}$ to the unit square in $\mathbb{R}^{2}$.

Definition 4.3 Let $S=[0,1]^{2}$. The rescaled interface associated with a function $\phi^{N}: S_{N} \rightarrow$ $\mathbb{R}$ is the function $\bar{\phi}^{N}: S \rightarrow \mathbb{R}$ given by

$$
\bar{\phi}^{N}(s, t):=\frac{1}{N} \phi\left(s^{\prime} N, t^{\prime} N\right)
$$

where $s^{\prime}$ is the smallest integer multiple of $1 / N$ greater than $s$, and $t^{\prime}$ is defined similarly.

In Chapter 6 of [19], Funaki shows the asymptotics of stochastic interfaces with potentials satisfying the conditions in Proposition 4.1 may be given in terms of minimisers of variational problems. Furthermore, it is known that the asymptotic shapes of interfaces with the bead interaction potential have intimate relationships with models in free probability (see e.g. Metcalfe [30]). With these observations in mind, and in the event that Conjecture 4.2 holds, we are lead to further predict the following result about the asymptotic shape of a certain class of interfaces.

Conjecture 4.4 Let $\phi^{N}$ be a stochastic interface model with interaction potential either $V=$ bead or $V=\exp$, and weight functions of the form

$$
W_{(i, j)}^{N}(u)=\mathbb{1}_{i=j} \mu u+\mathbb{1}_{i=j=N} N f_{N}(u / N),
$$

where $f_{N}$ be a function satisfying $f_{N}(u) \rightarrow \infty \mathbb{1}_{u \notin A}$ as $N \rightarrow \infty$, and $\mu$ is a positive real number. Suppose further that if $Z_{N}$ is the partition function associated with the stochastic interface on $S_{N}$, then the sequence $\left\{Z_{N}\right\}$ satisfies $\frac{1}{N^{2}} \log Z_{N} \rightarrow E \in \mathbb{R}$.

Then the rescaled interface converges pointwise almost surely to a deterministic limitthat is, each $(s, t)$ in $S, \bar{\phi}^{N}(s, t)$ converges almost surely to a deterministic limit $\xi(s, t)$. Moreover, the limit function $\xi$ is the minimiser in $\mathcal{C}^{1}(S)$ of the functional $\mathcal{E}: \mathcal{C}^{1}(S) \rightarrow \mathbb{R}$ given by

$$
\mathcal{E}[v]:=\int_{S} \sigma^{V}(\nabla v(s, t)) d s d t+\mu \int_{0}^{1} v(s, s) d s+\infty \mathbb{1}_{v(1,1) \notin A}+E .
$$

Finally, the value of the functional at the minimiser is given by $\mathcal{E}[\xi]=0$. 


\subsection{Asymptotics of the Whittaker Measure}

Now we consider the implications of Conjecture 4.4 for the function $\varphi_{\mu}^{N}$ defined in (3.9) and appearing as a stochastic interface in Proposition 3.2, with a particular focus on what this tells us about large- $N$ asymptotics of the partition functions $\tau_{\mu}^{N}(m, k)$ under the scaling $m=c N, k=\alpha N$.

Indeed, we anticipate that the rescaled interface $\bar{\varphi}_{\mu}^{N}$ converges pointwise almost-surely to a deterministic limit $\xi_{\mu}$, and that $\xi_{\mu}$ is the minimiser over all functions $v$ in $\mathcal{C}^{1}(S)$ satisfying $v(1,1) \geq 0$ of the energy functional $\mathcal{E}_{\mu}: \mathcal{C}^{1}(S) \rightarrow \mathbb{R}$

$$
\mathcal{E}_{\mu}[v]:=\int_{S} \sigma^{\exp }(\nabla v(s, t)) d s d t+\mu \int_{0}^{1} v(s, s) d s+\log \Gamma(\mu) .
$$

In this case, we are able to recover the large- $N$ asymptotics of the many-path partition functions associated with the inverse gamma polymer of parameter $\mu$. Namely, the large- $N$ asymptotics of (3.10) suggest that

$$
\lim _{N \rightarrow \infty} \frac{1}{N^{2}} \log \tau_{\mu}^{N}(c N, \alpha N):=\int_{0}^{\alpha} \xi_{\mu}(u, 1-c+u) d u .
$$

Short of offering an explicit expression for the limit shape $\xi_{\mu}$, we make a few predictions about its properties. First of all, by the symmetry of the energy functional $\mathcal{E}_{\mu}(4.3)$, it is plain that the minimiser $\xi_{\mu}$ is itself symmetric.

Moreover, consider minimising the two competing terms appearing in $\mathcal{E}_{\mu}[v]$ over functions satisfying $v(1,1)=0$. On the one hand, the first term $\int_{S} \sigma^{\exp }(\nabla v)$ encourages $v$ to have negative derivatives with respect to both $s$ and $t$, where as the intermediate term $\mu \int_{0}^{1} v(s, s) d s$ wants $v$ to decreases. As $\mu$ becomes larger, this second effect becomes stronger, and we anticipate that

$$
\xi_{\mu} \text { is monotone decreasing in } \mu .
$$

With these broad observations made, we now turn to discussing existing results in the literature which give us the values of $\xi_{\mu}$ on the boundaries of the square $S$. First we note that by (3.9) the values taken by $\varphi_{\mu}^{N}$ on the boundary of $S_{N}$ are given in terms of the one-path logarithmic partition functions

$$
\varphi_{\mu}^{N}(1, j):=\log \tau_{\mu}^{N}(N-j+1,1),
$$

which suggests

$$
\xi_{\mu}(0, t)=\lim _{N \rightarrow \infty} \frac{1}{N} \log \tau_{\mu}^{N}(N(1-t), 1) .
$$

In particular, by Seppäläinen's equation (2.9) we have

$$
\xi_{\mu}(0, t):=-\sup _{\theta \in[0, \mu]}\left((1-t) \psi_{0}(\theta)+\psi_{0}(\mu-\theta)\right),
$$

where $\psi_{0}(\mu):=\Gamma^{\prime}(\mu) / \Gamma(\mu)$ is the digamma function. Of course, by symmetry $\xi_{\mu}(t, 0)$ also satisfies (4.6).

We now seek to understand the values taken by $\xi_{\mu}$ on the other boundaries of the square $S$, namely points of the form $(t, 1)$ and $(1, t)$. To this end, again by (3.9) we have

$$
\varphi_{\mu}^{N}(i, N):=\log \left(\frac{\tau_{\mu}^{N}(i, i)}{\tau_{\mu}^{N}(i, i-1)}\right) .
$$


O'Connell and Ortmann study a random variable related to $\frac{\tau_{\mu}^{N}(i, i)}{\tau_{\mu}^{N}(i, i-1)}$ in [35]. In particular, it follows from Eq. (2.1) and Proposition 2.1 of [35] that we have the relation

$$
\tau_{\mu}^{N}(i, i) / \tau_{\mu}^{N}(i, i-1)=1 / Z_{\mu}^{\mathrm{OO}}(n, m),
$$

where for the choice $m=N-i+1$ and $n=i$, and $Z_{\mu}^{\mathrm{OO}}(n, m)$ is the partition function appearing in the introduction of [35]. Paraphrasing [35, Theorem 1.1], we have

$$
\frac{1}{t N} \log Z_{N-t N+1, t N}^{\mathrm{OO}} \rightarrow \inf _{\theta>0}\left(\frac{1}{t} \psi_{0}(\theta+\mu)-\psi_{0}(\theta)\right) .
$$

(We note here that this result was also obtained independently by Corwn et al. [15].) Combining (4.7) and (4.8) with the definition of $\xi_{\mu}(t, 1):=\lim _{N \rightarrow \infty} \frac{1}{N} \varphi_{\mu}^{N}(t N, N)$ and using (3.9), we obtain

$$
\xi_{\mu}(t, 1)=\sup _{\theta>0}\left(t \psi_{0}(\theta)-\psi_{0}(\mu+\theta)\right) .
$$

As a consistency check we remark that Eqs. (4.6) and (4.9) agree that $\xi_{\mu}(0,1)=-\psi_{0}(\mu)$.

Finally, we now show that the average of $\xi_{\mu}(s, t)$ along every diagonal $\{(u, 1-c+u)$ : $u \in[0, c]\}$ is given by $-\psi_{0}(\mu)$. Namely, we show

$$
\frac{1}{c} \int_{0}^{c} \xi_{\mu}(u, 1-c+u) d u=-\psi_{0}(\mu) .
$$

To see that (4.10) holds, we note from (3.8) that $\tau_{\mu}^{N}(m, m):=\prod_{i=1}^{m} \prod_{j=1}^{N} \zeta_{\mu}(i, j)$ is simply a product of $m N$ independent inverse-gamma random variables with parameter $\mu$, and it follows from the law of large numbers that when $m=c N$, we have the almost sure convergence

$$
\lim _{N \rightarrow \infty} \frac{1}{N^{2}} \log \tau_{\mu}^{N}(c N, c N)=c \mathbb{E}\left[\log \zeta_{\mu}(1,1)\right] .
$$

Now note that

$$
\mathbb{E}\left[\log \zeta_{\mu}(1,1)\right]=\int_{0}^{\infty} \log s \frac{s^{-\mu-1} e^{-1 / s} d s}{\Gamma(\mu)}=-\frac{\Gamma^{\prime}(\mu)}{\Gamma(\mu)}=-\psi_{0}(\mu) .
$$

Combining (4.11) and (4.12) with (4.4), we yield (4.10).

\subsection{The Small- $\mu$ Asymptotics of $\xi_{\mu}$ and the Marcenko-Pastur Law}

We now consider the asymptotics of the limit shape $\xi_{\mu}: S \rightarrow \mathbb{R}$ as $\mu \rightarrow 0$. First we recall the Marčenko-Pastur law concerning the asymptotic positions of eigenvalues of Laguerre matrices.

Suppose we have an $m \times m$ Laguerre distributed random matrix with underlying parameter $N$, and consider the asymptotics of the eigenvalues under the scaling limit $m=c N$ as $N \rightarrow \infty$. The Marčenko-Pastur law states that the empirical measure of the $m$ eigenvalues of the rescaled matrix $\frac{1}{N} H$ converges almost surely to the Marčenko-Pastur distribution $v_{c}$ with parameter $c$, where

$$
v_{c}(d u):=\frac{1}{2 \pi c} \frac{\sqrt{\left(M_{c}-u\right)\left(u-m_{c}\right)}}{u} \mathbb{1}_{u \in\left[m_{c}, M_{c}\right]} d u,
$$

and $m_{c}=1+c-2 \sqrt{c}$ and $M_{c}=1+c+2 \sqrt{c}$. (There is also a version of the Marčenko-Pastur distribution for $c>1$ which we don't consider.) 
We restate this result in terms of a position function $\rho_{\mathrm{mp}}: S \rightarrow \mathbb{R}$ as follows. Let $m=\lfloor c N\rfloor$ and $k=\lfloor\alpha N\rfloor$ with $0<\alpha \leq c \leq 1$, and define the random variable $\rho^{N}(c, \alpha)$ to be the the $k^{\text {th }}$ largest eigenvalue of a random matrix $\frac{1}{N} H$, where $H$ has law $P_{\mathrm{LUE}, m}^{N}$. Then as $N \rightarrow \infty, \rho^{N}(c, \alpha)$ converges almost surely $\rho(c, \alpha)$, the solution of the equation

$$
\int_{\rho_{\mathrm{mp}}(c, \alpha)}^{M_{c}} \frac{1}{2 \pi c} \frac{\sqrt{\left(M_{c}-u\right)\left(u-m_{c}\right)}}{u} \mathbb{1}_{u \in\left[m_{c}, M_{c}\right]} d u=\frac{\alpha}{c} .
$$

Taking $c=1-t+s$ and $\alpha=s$, and using the definition of the eigenvalue process $\varphi_{\mathrm{LUE}}^{N}$, we yield the following proposition .

Proposition 4.5 Let $\bar{\varphi}_{\mathrm{LUE}}^{N}: S \rightarrow \mathbb{R}$ be the rescaled interface associated with the Laguerre eigenvalue process. Then for each $(s, t)$ in $S, \bar{\varphi}_{\mathrm{LUE}}^{N}(s, t)$ converges almost surely to $\xi_{\mathrm{mp}}(s, t)$, where $\xi_{\mathrm{mp}}: S \rightarrow \mathbb{R}$ is the symmetric function satisfying

$$
\xi_{\mathrm{mp}}(s, t):=\rho_{\mathrm{mp}}(1-t+s, s) \text { for } s \leq t .
$$

On the other hand, assuming Conjecture 4.4, the representation of the eigenvalue process $\varphi_{\text {LUE }}^{N}$ as an interface in (3.4) implies that the asymptotic limit $\xi_{\mathrm{mp}}$ is the minimiser of the energy functional

$$
\mathcal{E}_{\mathrm{mp}}[v]:=\int_{S} \sigma^{\text {bead }}(\nabla v(s, t)) d s d t+\int_{0}^{1} v(s, s) d s+\infty \mathbb{1}_{v(1,1)<0} .
$$

Reiterating the connection with the interface $\varphi_{\mu}^{N}$, Theorem 4.5 can be read as saying

$$
\xi_{\mathrm{mp}}=\lim _{N \rightarrow \infty} \bar{\varphi}_{\mathrm{LUE}}^{N}=\lim _{N \rightarrow \infty} \lim _{\mu \rightarrow 0} \varphi_{\mu}^{N},
$$

where the convergence is pointwise almost sure on $S$. Assuming we can interchange the order of taking the limits $\mu \rightarrow 0$ and $N \rightarrow \infty$ in the final term of (4.15), we anticipate that

$$
\mu \xi_{\mu} \rightarrow \xi_{\mathrm{mp}} \text { as } \mu \rightarrow 0 .
$$

Finally, we now show that Proposition 4.5 implies Rost's equation (2.8) for the asymptotics associated with last passage percolation on an exponential polymer. Namely, taking $\mu \downarrow 0$ in (4.5), using (4.16) and (3.15) with $k=1$, we obtain

$$
\xi_{\mathrm{mp}}(0, t):=\ell_{1-t},
$$

where $\ell_{c}$ is defined as in (2.7). It remains to note from (4.14) and (4.13) that

$$
\ell_{c}=\xi_{\mathrm{mp}}(0,1-c)=\rho_{\mathrm{mp}}(c, 0)=M_{c}=(1+\sqrt{c})^{2},
$$

as required.

\subsection{The Large- $\mu$ Asymptotics of $\xi_{\mu}$}

We now consider the implications of our discussion in Sect. 3.4 as $N \rightarrow \infty$. Let $\theta_{\min }^{N}: S_{N} \rightarrow$ $\mathbb{R}$ be the function defined in (3.19), and let $\bar{\theta}_{\min }^{N}: S \rightarrow \mathbb{R}$ be the associated rescaling. By using Stirling's formula with the definition (3.19) of $\theta_{\mathrm{min}}^{N}$, it is possible to prove the following result. We provide details in Sect. 7.1. 
Theorem 4.6 Let $q(u)=u \log u$. The limit $\xi_{\mathrm{ht}}(s, t):=\lim _{N \rightarrow \infty} \bar{\theta}_{\min }^{N}(s, t)$ exists, and is given by the symmetric function satisfying

$$
\xi_{\text {ht }}(s, t)=q(s)+2 q(2-s-t)-q(2-t)-q(1-t)-q(1-s) \text { for } s \leq t .
$$

Again, assuming we can interchange the limits $N \rightarrow \infty$ and $\mu \rightarrow \infty$, by (3.18) we expect that

$$
\lim _{\mu \rightarrow \infty}\left(\xi_{\mu}(s, t)+(2-s-t) \log \mu\right)=\xi_{\text {ht }}(s, t) .
$$

Finally, in light of the fact that $\theta_{\mathrm{min}}^{N}$ minimises the energy functional (3.19), it is natural to expect the rescaled limit interface $\xi_{\text {ht }}: S \rightarrow \mathbb{R}$ is the minimiser of the energy functional

$$
\mathcal{E}_{\mathrm{ht}}[v]:=\int_{S} \sigma^{\mathrm{ht}}(\nabla v(s, t)) d s d t+\int_{0}^{1} v(s, s) d s+\infty \mathbb{1}_{v(1,1)<0},
$$

where $\sigma^{\text {ht }}(\nabla v(s, t))=\exp \left(\frac{\partial v}{\partial s}\right)+\exp \left(\frac{\partial v}{\partial t}\right)$. We regard the energy functional $\mathcal{E}_{\text {ht }}$ as an asymptotic analogue of the discrete functional in (3.20).

We take a moment to recapitulate on the large and small- $\mu$ limits of $\xi_{\mu}$ we have seen, and on their related variational problems. The function $\xi_{\mu}$, defined as the limit as $N \rightarrow \infty$ of the rescaled interface associated with $\varphi_{\mu}^{N}$, is the minimiser of the energy functional

$$
\int_{S} \sigma^{\exp }(\nabla v(s, t)) d s d t+\mu \int_{0}^{1} v(s, s) d s+\infty \mathbb{1}_{v(1,1)<0} .
$$

Anticipating that taking $\mu$ to either 0 or $\infty$ commutes with taking $N \rightarrow \infty$, we predicted the following. On the one hand, as $\mu \rightarrow 0$, we expect that $\mu \xi_{\mu}$ converges to $\xi_{\mathrm{mp}}$, which has an explicit expression (4.14) in terms of the Marcenko-Pastur distribution, and we predict is the minimiser of

$$
\int_{S} \sigma^{\text {bead }}(\nabla v(s, t)) d s d t+\int_{0}^{1} v(s, s) d s+\infty \mathbb{1}_{v(1,1)<0 .}
$$

On the other hand, as $\mu \rightarrow \infty$, we expect that $\xi_{\mu}(s, t)+(2-s-t) \log \mu$ converges to $\xi_{\text {ht }}$, which has the explicit expression (3.19) above, and we predict is the minimiser of

$$
\int_{S} \sigma^{\mathrm{ht}}(\nabla v(s, t)) d s d t+\int_{0}^{1} v(s, s) d s+\infty \mathbb{1}_{v(1,1)<0} .
$$

\subsection{Gaussian Fluctuations at High Temperature}

In the last section we looked at taking $\mu \rightarrow \infty$ after $N \rightarrow \infty$, anticipating the convergence to a limit shape. In this section, we develop a finer picture, taking $\mu$ and $N$ to infinity together through the scaling limit $\mu=\kappa N^{2}$, and using the central limit theorem to characterise the fluctuations of $\tau_{\mu}^{N}(m, k)$ in this regime.

Let $Q_{m, k}^{N}$ be the uniform law on $\Gamma^{N}(m, k)$, and let $\pi$ be a random variable with law $Q_{m, k}^{N}$. We make several assumptions about the asymptotic density of scaled $k$-paths in the $N \times N$ square. First, for $(u, v) \in S$ and $\alpha \leq c \leq 1$, we expect the existence of the limit

$$
\lim _{N \rightarrow \infty} Q_{c N, \alpha N}^{N}((u N, v N) \in \pi)=: q_{c, \alpha}(u, v) .
$$


Moreover, for any $n$ distinct points $\left(u_{1}, v_{1}\right), \ldots,\left(u_{n}, v_{n}\right)$ in $S$ we anticipate that we have the asymptotic decoupling

$$
\lim _{N \rightarrow \infty} Q_{c N, \alpha N}^{N}\left(\left\{\left(u_{1}, v_{1}\right), \ldots,\left(u_{n}, v_{n}\right)\right\} \in \pi\right)=\prod_{i=1}^{n} q_{c, \alpha}\left(u_{i}, v_{i}\right) .
$$

This decoupling equation (4.19) can be seen to hold, at least in a weak sense, via the wellknown bijection between non-intersecting paths and lozenge tilings of a hexagon, and in fact one can write down an explicit formula for the limiting function $q_{c, \alpha}(u, v)$ via a formula given for the limit shape of the corresponding tiling model in [8]. We also refer the reader to the work of Johansson [23], where the non-intersecting paths model is related to an extended Hahn process.

Now let $\kappa>0$, and consider the sequence of random processes $H_{\kappa}^{N}:=\left\{H_{\kappa}^{N}(s, t): 0 \leq\right.$ $s \leq t \leq 1\}$ given by

$$
H_{\kappa}^{N}(s, t):=k(N+m-k)(\log \kappa+2 \log N)+\log \tau_{\kappa N^{2}}^{N}(m, k)-\log \# \Gamma^{N}(m, k),
$$

where $m=\lfloor t N\rfloor$ and $k=\lfloor s N\rfloor$. In Section 7.2, we sketch ideas leading us to the following conjecture.

Conjecture 4.7 The limit (4.18) exists and the satisfies the asymptotic decoupling (4.19). Furthermore, the random process $\left\{H_{\kappa}^{N}(s, t): 0 \leq s \leq t \leq 1\right\}$ converges in distribution to a centred Gaussian process $\left\{H_{\kappa}(s, t)\right\}$ with covariance

$$
\mathbb{E}\left[H_{\kappa}(s, t) H_{\kappa}(\hat{s}, \hat{t})\right]=\frac{1}{\kappa}\left\langle q_{t, s}, q_{\hat{t}, \hat{s}}\right\rangle_{L^{2}(S)},
$$

where $\langle f, g\rangle_{L^{2}(S)}:=\int_{S} f(u, v) g(u, v) d u d v$ is the $L^{2}$ inner product on the unit square $S$.

It is immediate from (4.18) that $q_{c, c}(u, v)=\mathbb{1}_{v<c}$ in $L^{2}(S)$, and in particular, for any $t, \hat{t}$ in $[0,1]$ we have $\left\langle q_{t, t}, q_{\hat{t}, \hat{t}}\right\rangle=t \wedge \tilde{t}$. This observation implies that

$$
\left\{\sqrt{\kappa} H_{\kappa}(t, t): 0 \leq t \leq 1\right\} \text { is a Brownian motion, }
$$

though we mention here that is is possible to deduce (4.20) more directly by applying Donsker's invariance principle to the collection of random variables $\left\{\tau_{\kappa N^{2}}^{N}(c N, c N): c \in\right.$ $[0,1]\}$.

\subsection{The Semicircle Law and Surface Tension in the Bead Model}

In Sect. 8 we combine tools from random matrix theory with our variational approach to macroscopics of stochastic interfaces to obtain the following explicit expression for the surface tension associated with bead model

$$
\sigma_{\text {tilted }}^{\text {bead }}(p, q):= \begin{cases}C-\log \left(|p| \cos \left(\pi \frac{q}{|p|}\right)\right) & \text { if } p<0,|q|<\frac{1}{2}|p| \\ \infty & \text { otherwise, }\end{cases}
$$

where $C$ is a constant independent of $p$ and $q$, and $\sigma_{\text {tilted }}^{\text {bead }}$ is the surface tension in the change of coordinates given by $\sigma_{\text {tilted }}^{V}(p, q):=\sigma^{V}\left(\frac{1}{2} p-q, \frac{1}{2} p+q\right)$.

An equivalent formula to (4.21) was obtained in Sun [43], where it is proved by viewing the bead model as a continuous version of the Cohn-Kenyon-Propp [9] dimer model. Sun uses Kasteleyn theory [25] to express the partition functions of this dimer model in terms 
of a contour integral, and studies the asymptotics of these contour integrals to obtain the expression [43, Definition 5.4].

Our approach, which we now overview, uses simpler technology from variational analysis and random matrix theory. On the one hand, we have the following result concerning the asymptotics of $\varphi_{\mathrm{GUE}}^{N}$, which is simply a restatement of the semicircle law of classical random matrix theory [1].

Theorem 4.8 The rescaled interface $\bar{\varphi}_{\mathrm{GUE}}^{N}$ associated with the eigenvalue process of a GUE random matrix converges pointwise almost surely to a symmetric function $\xi_{\mathrm{sc}}: S \rightarrow \mathbb{R}$ satisfying

$$
\xi_{\mathrm{sc}}(s, t):=\sqrt{1-t+s} \rho_{\mathrm{sc}}\left(\frac{s}{1-t+s}\right) \quad \text { for } \quad s \leq t,
$$

where $\rho_{\mathrm{sc}}(x) \in[-2,2]$ is defined implicitly through the equation

$$
\int_{\rho_{\mathrm{sc}}(x)}^{2} \frac{1}{2 \pi} \sqrt{4-u^{2}} d u=x .
$$

On the other hand, assuming the truth of a result analogous to Conjecture 4.4-with quadratic weight functions $\mathbb{1}_{i=j} \frac{u^{2}}{2}$ in place of the linear weight functions $\mathbb{1}_{i=j} \mu u$ occuring in (4.2) — we expect that the limit $\xi_{s c}$ is the minimiser of the energy functional

$$
\mathcal{E}_{\mathrm{sc}}[v]:=\int_{S} \sigma^{\text {bead }}(\nabla v(s, t)) d s d t+\frac{1}{2} \int_{0}^{1} v(s, s)^{2} d s .
$$

With these observations at hand, the main idea of our derivation is straightforward: if the minimiser of (4.23) has the form (4.22), then the tilted surface tension of the bead model must be given by (4.21).

To sketch out a few further steps here, we develop the following scaling limit of the formula (3.3) for the volume of the Gelfand-Tsetlin polytope. Namely, suppose we have a strictly decreasing $\rho:[0,1] \rightarrow \mathbb{R}$. Then taking $N \rightarrow \infty$ in the definition of the Vandermonde determinant $\Delta_{N}(\lambda):=\prod_{1 \leq i<j \leq N}\left(\lambda_{i}-\lambda_{j}\right)_{+}$, we obtain

$$
\begin{aligned}
\Delta[\rho] & :=\lim _{N \rightarrow \infty}\left(\frac{1}{N^{2}} \log \Delta_{N}(N \rho(1 / N), \ldots, N \rho(N / N))-\frac{1}{2} \log N\right) \\
& =\int_{0<s<t<1} \log (\rho(s)-\rho(t)) d s d t .
\end{aligned}
$$

Moreover, the large- $N$ asymptotics of the superfactorial are given by

$$
\log H(N)=N^{2}\left(\frac{1}{2} \log N-\frac{3}{4}+o(1)\right)
$$

(See e.g. Chen [7].) Combining (4.24) and (4.26), we see that on the one hand, since $g^{\text {bead }}(\lambda)=\Delta_{N}(\lambda) / H(N)$,

$$
\lim _{N \rightarrow \infty} \frac{1}{N^{2}} \log g^{\text {bead }}(N \rho(1 / N), \ldots, N \rho(N, N))=\int_{0<s<t<1} \log (\rho(s)-\rho(t)) d s d t+\frac{3}{4} .
$$


On the other hand, reading directly off the integral representation of the pattern integral $g$ bead as it appears in (3.3), it is natural to expect that as defined,

$$
\lim _{N \rightarrow \infty} \frac{1}{N^{2}} \log g^{\text {bead }}(N \rho(1 / N), \ldots, N \rho(N, N))=-\min _{v \in \mathcal{C}^{1}(T)_{\rho}} \int_{T} \sigma^{\text {bead }}(\nabla v),
$$

where $T:=\{0 \leq s \leq t \leq 1\}$, and $\mathcal{C}^{1}(T)_{\rho}$ is the set of $\mathcal{C}^{1}$ functions on $T$ satisfying $v(s, s)=\rho(s)$ for all $s \in[0,1]$.

Combining (4.27) with (4.28), we obtain the following thermodynamic analogue of the Gelfand-Tsetlin volume formula

$$
\mathcal{M}^{V}[\rho]:=\min _{v \in \mathcal{C}^{1}(T)_{\rho}} \int_{T} \sigma^{\text {bead }}(\nabla v)=-\int_{0<s<t<1} \log (\rho(s)-\rho(t)) d s d t-\frac{3}{4} .
$$

We consider the functional derivatives of the functional $\mathcal{M}^{V}[\rho]$, showing that if $v^{*}$ is the minimiser of the integral over $T$ in (4.29), then $v^{*}$ satisfies the equation

$$
\frac{\partial \sigma}{\partial \tau}\left(\frac{\partial v^{*}}{\partial r}(r, 0), \frac{\partial v^{*}}{\partial \tau}(r, 0)\right)=\int_{0}^{1} \frac{1}{\rho(r)-\rho(s)} d s .
$$

However, we also know that $v^{*}$ must also be related to the semicircle law as it appears in (4.22). By plugging $\xi_{s c}$ into the differential equation (4.30), and using a homogeneityproperty of the surface tension, we determine $\sigma_{\text {tilted }}^{\text {bead }}$ as having the form in (4.21). The full details of this argument are given in Sect. 8, though it bares remarking here that in principle we could have alternatively used the Marčenko-Pastur distribution in place of the semicircle law, and we only opt to use the latter because the calculations involved are more straightforward.

\section{The Finite $k$ Case}

This section is dedicated to proving the results stated in Sect. 2. In Sect. 5.1 we prove Lemma 2.7, which relates the partition function $Z_{\mathbf{x} \rightarrow \mathbf{y}}(\beta)$ to its infinite temperature counterpart, $Z_{\mathbf{x} \rightarrow \mathbf{y}}(0)$. Sects. 5.3 and 5.4 are dedicated to giving proofs of Theorems 2.1 and 2.2 concerning the asymptotics of the partition functions associated with a fixed number of long non-intersecting paths. In Sect. 5.5 we prove Theorem 2.4 and in Section 5.6 we use Szegö's limit theorem to prove Theorem 2.5. Finally, in Sect. 5.7 we give a derivation of Macmahon's formula leading to a proof of Lemma 2.6.

We will use the following notation. If $\left(X_{n}\right)_{n \geq 1}$ are random variables and $\theta$ is a real number, we will write

$$
X_{n} \rightarrow \theta \quad \text { a.s.e } \quad \text { as } n \rightarrow \infty
$$

to denote that the sequence $X_{n}$ converges both almost-surely and in expectation to $\theta$ as $n \rightarrow \infty$. Namely,

$$
\mathbb{P}\left(X_{n} \rightarrow \theta\right)=1 \quad \text { and } \quad \mathbb{E}\left[\left|X_{n}-\theta\right|\right] \rightarrow 0 \quad \text { as } n \rightarrow \infty
$$




\subsection{The Infinite Temperature Sandwich Bounds}

Recall that $\varpi(\mathbf{x}, \mathbf{y}):=\sum_{i=1}^{k}\left\|y_{i}-x_{i}\right\|_{1}, v:=\mathbb{E}[\omega(0,0)]$, and $G(\beta):=\mathbb{E}\left[e^{\beta \omega(0,0)}\right]$. We now use Jensen's inequality to prove Lemma 2.7 , which states that we have the bounds

$$
\beta v \leq \frac{1}{\varpi(\mathbf{x}, \mathbf{y})} \mathbb{E} \ln \frac{Z_{\mathbf{x} \rightarrow \mathbf{y}}(\beta)}{Z_{\mathbf{x} \rightarrow \mathbf{y}}(0)} \leq \log G(\beta) .
$$

Proof of Lemma 2.7 The infinite temperature partition function $Z_{\mathbf{x} \rightarrow \mathbf{y}}(0)$ counts the set $\Gamma_{\mathbf{x} \rightarrow \mathbf{y}}$ of $k$-paths from $\mathbf{x} \rightarrow \mathbf{y}$. Let $Q_{\mathbf{x} \rightarrow \mathbf{y}}$ be the uniform measure on $\Gamma_{\mathbf{x} \rightarrow \mathbf{y}}$, and let $\Pi$ be a k-pathvalued random variable with law $Q_{\mathbf{x} \rightarrow \mathbf{y}}$. By definition,

$$
\frac{Z_{\mathbf{x} \rightarrow \mathbf{y}}(\beta)}{Z_{\mathbf{x} \rightarrow \mathbf{y}}(0)}=Q_{\mathbf{x} \rightarrow \mathbf{y}}\left[e^{\beta F(\Pi)}\right],
$$

where $F(\pi):=\sum_{i=1}^{k} \sum_{x \in \pi_{i}-\left\{x_{i}\right\}} \omega(x)$. In particular,

$$
\frac{1}{\varpi(\mathbf{x}, \mathbf{y})} \mathbb{E} \ln \frac{Z_{\mathbf{x} \rightarrow \mathbf{y}}(\beta)}{Z_{\mathbf{x} \rightarrow \mathbf{y}}(0)}=\frac{1}{\varpi(\mathbf{x}, \mathbf{y})} \mathbb{E} \ln Q_{\mathbf{x} \rightarrow \mathbf{y}}\left[e^{\beta F(\Pi)}\right] .
$$

Under the measure $\mathbb{P} Q_{\mathbf{x} \rightarrow \mathbf{y}}, F(\Pi)$ is equal in law to the sum of $\varpi(\mathbf{x}, \mathbf{y})$ independent and identically distributed random variables with law $\omega(0,0)$.

Now on the one hand, using Jensen's inequality to interchange the order of $\mathbb{E}$ and ln, we obtain

$$
\begin{aligned}
\frac{1}{\varpi(\mathbf{x}, \mathbf{y})} \mathbb{E} \ln Q_{\mathbf{x} \rightarrow \mathbf{y}}\left[e^{\beta F(\Pi)}\right] & \leq \frac{1}{\varpi(\mathbf{x}, \mathbf{y})} \ln \mathbb{E} Q_{\mathbf{x} \rightarrow \mathbf{y}}\left[e^{\beta F(\Pi)}\right] \\
& =\frac{1}{\varpi(\mathbf{x}, \mathbf{y})} \ln \left(G(\beta)^{\varpi(\mathbf{x}, \mathbf{y})}\right),
\end{aligned}
$$

yielding the upper bound in (5.1). On the other hand, using Jensen's inequality to interchange the order of $\ln$ and $Q_{\mathbf{x} \rightarrow \mathbf{y}}$ we have

$$
\begin{aligned}
\frac{1}{\varpi(\mathbf{x}, \mathbf{y})} \mathbb{E} \ln Q_{\mathbf{x} \rightarrow \mathbf{y}}\left[e^{\beta F(\Pi)}\right] & \geq \frac{1}{\varpi(\mathbf{x}, \mathbf{y})} \mathbb{E} Q_{\mathbf{x} \rightarrow \mathbf{y}}\left[\ln e^{\beta F(\Pi)}\right] \\
& =\frac{1}{\varpi(\mathbf{x}, \mathbf{y})}(\varpi(\mathbf{x}, \mathbf{y}) \nu \beta),
\end{aligned}
$$

giving the lower bound in (5.1).

In a later proof, we will require the following blunt lower bound on $\mathbb{E}\left[\log Z_{\mathbf{x} \rightarrow \mathbf{y}}(\beta)\right]$, which is an immediate corollary of (5.1).

Corollary 5.1 Let $\mathbf{x} \leq \mathbf{y}$. Then

$$
\beta \nu \varpi(\mathbf{x}, \mathbf{y}) \leq \mathbb{E}\left[\log Z_{\mathbf{x} \rightarrow \mathbf{y}}(\beta)\right]
$$

Proof Since $\mathbf{x} \leq \mathbf{y}, \log Z_{\mathbf{x} \rightarrow \mathbf{y}}(0) \geq 0$. Now rearrange the lower bound in (5.1).

\subsection{Diagonal $k$-Points}

For technical reasons, in our proofs of asymptotic results we will like to use a particular type of nice $k$-point with several desirable properties. Namely, for $x \in \mathbb{Z}^{2}$, define the diagonal 
$k$-point at $x^{\Uparrow k}=\left(x_{1}^{\Uparrow k}, \ldots, x_{k}^{\Uparrow k}\right)$ with base $x$ by

$$
x_{i}^{\Uparrow k}:=x+(i-1)\left(-\mathbf{e}_{1}+\mathbf{e}_{2}\right) .
$$

Recall that for 1-points $x=x_{1} \mathbf{e}_{1}+x_{2} \mathbf{e}_{2}$ and $y=y_{1} \mathbf{e}_{1}+y_{2} \mathbf{e}_{2}$ of $\mathbb{Z}^{2}$, we say $x \leq y$ if $x_{1} \leq y_{1}$ and $x_{2} \leq y_{2}$. We have $x \leq y$ if and only if there is a 1-path from $a$ to $b$. The following proposition gives us a useful property of diagonal $k$-points.

Proposition 5.2 Let $x$ and $y$ be 1-points of $\mathbb{Z}^{2}$. Then $x \Uparrow k \leq y \Uparrow k$ if and only if $x \leq y$.

Proof On the one hand, if $x \Uparrow k \leq y \Uparrow k$, then plainly there is a 1-path from $x=x_{1}^{\Uparrow k}$ to $y=y_{1}^{\Uparrow k}$. On the other hand, if $x \leq y$, then there exists a 1-path $\pi_{1}=\left\{x, z_{1}, \ldots, z_{p-1}, y\right\}$ from $x$ to $y$. Now for $i=2, \ldots, k$, define

$$
\begin{aligned}
\pi_{i}:= & \left\{x+(i-1)\left(-\mathbf{e}_{1}+\mathbf{e}_{2}\right), z_{1}+(i-1)\left(-\mathbf{e}_{1}+\mathbf{e}_{2}\right), z_{2}+(i-1)\left(-\mathbf{e}_{1}+\mathbf{e}_{2}\right), \ldots, y\right. \\
& \left.+(i-1)\left(-\mathbf{e}_{1}+\mathbf{e}_{2}\right)\right\} .
\end{aligned}
$$

Then $\pi=\left(\pi_{1}, \ldots, \pi_{k}\right)$ is a $k$-path from $x^{\Uparrow k}$ to $y^{\Uparrow k}$.

Proposition 5.3 Let $\mathbf{x}=\left(x_{1}, \ldots, x_{k}\right)$ be a k-point with strictly increasing vertical coordinates in the sense that writing $x_{i}=\left(a_{i}, b_{i}\right)$, we have $b_{1}<\ldots<b_{k}$. (In particular, all nice $k$-points have this property.) Then there exists a 1 -point $y_{*}$ in $\mathbb{Z}^{2}$ such that $y^{\Uparrow k} \geq \mathbf{x}$ for all $y \geq y_{*}$. Likewise there exists a 1 -point $z_{*}$ such that $z^{\Uparrow k} \leq \mathbf{x}$ whenever $z \leq z_{*}$.

Proof With $r:=\max \left\{a_{i}: 1 \leq i \leq k\right\}$, define $a_{i}^{\prime}:=r+k-i$ and $b_{i}^{\prime}=b_{i}$. Then there is a horizontal $k$-path travelling from $\mathbf{x}$ to $\mathbf{x}^{\prime}:=\left(x_{1}^{\prime}, \ldots, x_{k}^{\prime}\right)$ defined by $x_{i}^{\prime}:=\left(a_{i}^{\prime}, b_{i}^{\prime}\right)$, and hence $\mathbf{x} \leq \mathbf{x}^{\prime}$. Now define $s:=\max \left\{b_{i}: 1 \leq i \leq k\right\}$. Then there is a vertical $k$-path travelling from $\mathbf{x}^{\prime}$ to $(r+k-1, s-k+1)^{\Uparrow k}$. Setting $y_{*}:=(r+k-1, s-k+1)$, Proposition (5.2) ensures that $\mathbf{x} \leq y^{\Uparrow k}$ for all $y \geq y_{*}$. The proof of the existence of $z_{*}$ is similar.

\subsection{Proof of Theorem 2.1}

In this section we use a subadditivity argument to prove Theorem 2.1, which we recall states that there exists a function $f_{c}(k, \beta)$ such that for any pair of nice $k$-points $\mathbf{x}$ and $\mathbf{y}$, we have the almost sure convergence $\frac{1}{N} \log Z_{\mathbf{x} \rightarrow \mathbf{y}+(N, c N)}(\beta) \rightarrow f_{c}(k, \beta)$.

The main idea here is that by the series bound (1.3), the random variables $Y_{\mathbf{x} \rightarrow \mathbf{y}}:=$ $-\log Z[\mathbf{x} \rightarrow \mathbf{y}]$ are subadditive with respect to the partial ordering $\leq$ of nice points $\mathbb{Z}^{2 \times k}$ in the sense that for any three nice $k$-points $\mathbf{x} \leq \mathbf{y} \leq \mathbf{z}$, we have

$$
Y_{\mathbf{x} \rightarrow \mathbf{z}} \leq Y_{\mathbf{x} \rightarrow \mathbf{y}}+Y_{\mathbf{y} \rightarrow \mathbf{z}} .
$$

We want to exploit this subadditivity to study the asymptotics of the random variable $Z_{\mathbf{x} \rightarrow \mathbf{y}+(N, c N)}$ as $N$ tends to infinity. To do so, we recall Kingman's subadditive ergodic theorem [26], giving a slight restatement of a later version of the theorem by Liggett [28]. (In the statement in [28], the random variables are indexed over pairs of natural numbers, where as we allow our random variables to be indexed by the integers. It is not difficult to see the consequences of the theorem continue to hold in this setting.)

Theorem 5.4 Let $\left(X_{r, s}\right)_{r<s}$ be a family of random variables indexed over either $\{(r, s) \in$ $\left.\mathbb{Z}^{2}: r<s\right\}$ or $\left\{(r, s) \in \mathbb{Z}_{\geq 0}^{2}: r<s\right\}$ and satisfying the following three conditions.

1. For every triple of integers $r<s<t$, we have $X_{r, t} \leq X_{r, s}+X_{s, t}$. 
2. For every $a \geq 0$, the joint distribution of the processes $\left(X_{r+a, s+a}\right)_{0 \leq r<s}$ are the same as those of $\left(X_{r, s}\right)_{0 \leq r<s}$.

3. The expectation $g_{r}:=\mathbb{E}\left[X_{0, r}\right]$ exists, and there exists a real number $L$ such that $\frac{g_{r}}{r} \geq L$ for every $r \geq 1$.

Then there is a constant $\theta$ such that for every $r$ in $\mathbb{Z}$,

$$
X_{r, s} / s \rightarrow \theta \quad \text { a.s.e } \quad \text { as } s \rightarrow \infty .
$$

Moreover, for any $r, \theta=\inf _{s>0} \frac{1}{s} \mathbb{E}\left[X_{0, s}\right]$.

With Kingman's subadditive ergodic theorem now stated, the proof of Theorem 2.1 is split into three parts. First, in Lemma 5.5 we prove that the conclusion of Theorem 2.1 holds for rational $c$ and for all diagonal $k$-points situated on a certain lattice. Thereafter we prove Lemma 5.6, which gives us a pair of inequalities for the asymptotics of partition functions in a more general setting. Using Lemma 5.6, we deduce that the dependence on the parameter $c$ is continuous, and use this observation to prove that the conclusion of Theorem 2.1 holds for all nice $k$-points and all positive slopes $c$.

Lemma 5.5 Let $c>0$ be a rational number and let $p$ and $q$ be positive integers such that $q / p=c$. For integers $r \in \mathbb{Z}$ define the $k$-points $x_{r}:=(r p, r q)^{\Uparrow k}$, and for pairs of integers $r<s$, define the random variables

$$
X_{r, s}:=-\frac{1}{p} \log Z_{x_{r} \rightarrow x_{s}}(\beta) .
$$

Then there is a real number $f_{c}(k, \beta)$ such that for any $r$, the random variables $\frac{1}{s} X_{r, s}$ converge to $-f_{c}(k, \beta)$ a.s.e. as $s \rightarrow \infty$. Moreover $f_{c}(k, \beta)$ is independent of the choice of positive integers $p$ and $q$ satisfying $q / p=c$.

Proof First we show that with $X_{r, s}$ defined as in (5.4), we are in the set up of Theorem 5.4. First of all, the inequality $X_{r, t} \leq X_{r, s}+X_{s, t}$ is a consequence of (5.3). The second condition is immediate from the definition of $X_{r, s}$ (using the fact that the polymer weights are independent and identically distributed). Finally, the fact that the final condition is satisfied is a consequence of Lemma 2.7. Indeed, by using the upper bound in Lemma 2.7 to obtain the inequality below, we have

$$
\begin{aligned}
\mathbb{E}\left[X_{0, r}\right] & =-\frac{1}{p} \mathbb{E}\left[\log Z_{x_{0} \rightarrow x_{r}}(\beta)\right] \\
& \geq-\frac{1}{p}\left\{\varpi\left(x_{0}, x_{r}\right) \log G(\beta)+\mathbb{E}\left[\log Z_{x_{0} \rightarrow x_{r}}(0)\right]\right\} \\
& =-r k \frac{p+q}{p} \log G(\beta)-\frac{1}{p} \mathbb{E}\left[\log Z_{x_{0} \rightarrow x_{r}}(0)\right],
\end{aligned}
$$

where we used the fact that $\varpi\left(x_{0}, x_{r}\right)=r k(p+q)$ to obtain the final equality above. Now using the parallel bound (1.6) with $\beta=0$ to prove the first inequality below, and the inequality $\left(\begin{array}{l}b \\ a\end{array}\right) \leq\left(\frac{e b}{a}\right)^{a}$ to obtain the second, we have

$$
\begin{aligned}
\# \Gamma_{(0,0) \Uparrow k \rightarrow(r p, r q) \Uparrow k} & \leq k \# \Gamma_{(0,0) \rightarrow(r p, r q)}=k\left(\begin{array}{c}
r(p+q) \\
r p
\end{array}\right) \\
& \leq k\left(\frac{e(p+q)}{p}\right)^{r p}
\end{aligned}
$$


Plugging (5.6) into (5.5) we obtain

$$
\mathbb{E}\left[X_{0, r}\right] \geq-\frac{1}{p} \log k-r\left(-\log \left(\frac{p+q}{p}\right)-k \frac{p+q}{p} G(\beta)-1\right),
$$

which proves the third and final condition holds in Theorem 5.4.

Consequently, by Theorem 5.4, for each $r \in \mathbb{Z}$, the limit

$$
f_{p, q}(k, \beta):=-\lim _{s \rightarrow \infty} \frac{1}{s} X_{r, s}=\frac{1}{p} \lim _{s \rightarrow \infty} \frac{1}{s} \log Z_{x_{r} \rightarrow x_{s}}(\beta)
$$

exists almost-surely as a constant not depending on $r$.

It remains to prove $f_{p, q}(k, \beta)$ is independent of the choice of $p$ and $q$. To see this, first note that we may assume without loss of generality that $r=0$ since $f_{p, q}(k, \beta)$ is independent of $r$. In this case writing $x_{r}^{p, q}:=(r p, r q)^{\Uparrow k}$ to emphasise the dependence on $p$ and $q$, on the one hand the random variables $\left(W_{r}^{p, q}\right)_{r \geq 1}$ given by

$$
W_{r}^{p, q}:=-\frac{1}{r} X_{0, r}=\frac{1}{r p} \log Z_{x_{0}^{p, q} \rightarrow x_{r}^{p, q}}(\beta)
$$

converge almost surely to $f_{p, q}(k, \beta)$ as $r \rightarrow \infty$. Now suppose $p^{\prime}$ and $q^{\prime}$ are another pair of integers such that $q^{\prime} / p^{\prime}=c$. Then it is immediate that $p^{\prime} q=q^{\prime} p$, and hence for every $r$

$$
W_{p^{\prime} r}^{p, q}=W_{p r}^{p^{\prime}, q^{\prime}}
$$

and in particular, almost-surely we have

$$
f_{p, q}(k, \beta)=\lim _{r \rightarrow \infty} W_{p^{\prime} r}^{p, q}=\lim _{r \rightarrow \infty} W_{p r}^{p^{\prime}, q^{\prime}}=f_{p^{\prime}, q^{\prime}}(k, \beta),
$$

This ensures that $f_{p, q}(k, \beta)$ is independent of the choice of $p$ and $q$, and justifies us hereafter writing $f_{c}(k, \beta)$ for this quantity.

In Lemma 5.5, for rational values of $c$ we defined $f_{c}(k, \beta)$ as a limit associated with the partition functions at a slope of $c$. The main remaining step in the proof of Theorem 2.1 is the following lemma, which provides upper and lower bounds on the asymptotic growth of partition functions associated with $k$-points across $\left(1, c^{\prime}\right)$ asymptotics-where $c^{\prime}$ is any positive number-in terms of those associated with diagonal points at rational tilts $c$.

Lemma 5.6 Let $\mathbf{x}$ and $\mathbf{y}$ be nice $k$-points, and let c and $c^{\prime}$ be positive reals such that $0<c<c^{\prime}$ and $c$ is rational. Then we have

$$
\lim \inf _{N \rightarrow \infty} \frac{1}{N} \log Z_{\mathbf{x} \rightarrow \mathbf{y}+\left(N, c^{\prime} N\right)}(\beta) \geq f_{c}(k, \beta)+k\left(c^{\prime}-c\right) \beta v \quad \text { almost-surely, }
$$

and

$$
\lim \sup _{N \rightarrow \infty} \frac{1}{N} \log Z_{\mathbf{x} \rightarrow \mathbf{y}+\left(N, c^{\prime} N\right)}(\beta) \leq \frac{c^{\prime}}{c} f_{c}(k, \beta)-k\left(\frac{c^{\prime}}{c}-1\right) \beta v \quad \text { almost-surely. }
$$

Proof First we prove Eq. (5.8). Since the distribution of the random variable $\log Z_{\mathbf{x} \rightarrow \mathbf{y}+\left(N, c^{\prime} N\right)}(\beta)$ is invariant under translations $(\mathbf{x}, \mathbf{y}) \mapsto(\mathbf{x}+a, \mathbf{y}+a)$, without loss of generality we may assume that $0^{\Uparrow k} \leq y$. Suppose $p$ and $q$ are positive integers such that $q / p=c$ and recall the notation

$$
x_{r}:=(r p, r q)^{\Uparrow k}
$$


Since $\mathbf{x}$ is nice, by Proposition 5.3 there is a sufficiently large integer $r \in \mathbb{Z}$ such that $x_{r} \geq \mathbf{x}$. Now for each $N>0$, we may write

$$
N=\alpha_{N} p+\beta_{N}
$$

where $\alpha_{N}$ and $\beta_{N}$ are non-negative integers and $\beta_{N} \in\{0,1, \ldots, p-1\}$. Let $N$ be such that $\alpha_{N} \geq r$, and consider the chain of nice $k$-points

$$
\mathbf{x} \leq x_{r} \leq x_{\alpha_{N}} \leq\left(N, \alpha_{N} q\right)^{\Uparrow k} \leq\left(N, c^{\prime} N\right)^{\Uparrow k} \leq \mathbf{y}+\left(N, c^{\prime} N\right),
$$

where the fact that $\left(N, c^{\prime} N\right)^{\Uparrow k} \leq \mathbf{y}+\left(N, c^{\prime} N\right)$ is a consequence of translating the inequality $(0,0)^{\Uparrow k} \leq \mathbf{y}$. By applying the series bound to (5.10) we have

$$
\frac{1}{N} \log Z_{\mathbf{x} \rightarrow \mathbf{y}+\left(N, c^{\prime} N\right)}(\beta) \geq \frac{1}{N}\left(a+b_{N}+c_{N}+d_{N}+e_{N}\right)
$$

where

$$
\begin{aligned}
a & :=\log Z_{\mathbf{x} \rightarrow x_{r}}(\beta), \quad b_{N}:=\log Z_{x_{r} \rightarrow x_{\alpha_{N}}}(\beta), \quad c_{N}:=\log Z_{x_{\alpha_{N}} \rightarrow\left(N, \alpha_{N} q\right)^{\Uparrow k}}(\beta) \\
d_{N} & :=\log Z_{\left(N, \alpha_{N} q\right) \Uparrow k \rightarrow\left(N, c^{\prime} N\right)^{\Uparrow k}}(\beta), \quad e_{N}:=\log Z_{\left(N, c^{\prime} N\right) \Uparrow k \rightarrow \mathbf{y}+\left(N, c^{\prime} N\right)}(\beta) .
\end{aligned}
$$

We now consider the asymptotic contribution coming from each of these five terms. First, we note that $a$ is independent of $N$, and hence

$$
\lim _{N \rightarrow \infty} \frac{1}{N} a=0 \quad \text { almost-surely. }
$$

Second, we note that we may write $b_{N}:=-p X_{r, \alpha_{N}}$, where $X_{r, s}$ is defined as Lemma 5.5. In particular, by Lemma 5.5 , as $N \rightarrow \infty$,

$\lim _{N \rightarrow \infty} \frac{1}{N} b_{N}=-\lim _{N \rightarrow \infty} \frac{1}{\alpha_{N} p+\beta_{N}} p X_{r, \alpha_{N}}=-\lim _{N \rightarrow \infty} \frac{1}{\alpha_{N}} X_{r, \alpha_{N}}=f_{c}(k, \beta) \quad$ almost-surely.

Now we consider the term $c_{N}$. Recalling that $x_{\alpha_{N}}:=\left(\alpha_{N} p, \alpha_{N} q\right)^{\Uparrow k}$, we note that $x_{\alpha_{N}}$ and $\left(N, \alpha_{N} q\right)^{\Uparrow k}$ have the same vertical coordinates. It follows that there is only one $k$-path in $\Gamma_{x_{\alpha_{N}} \rightarrow\left(N, \alpha_{N} q\right) \Uparrow k}$ - the $k$-path travelling horizontally. Moreover, each constituent 1-path in the $k$-path has length $N-\alpha_{N} p \in\{0,1, \ldots, p-1\}$. In particular, for each $N, c_{N}$ is simply a sum of at most $k(p-1)$ independent and identically distributed random variables. It follows that

$$
\lim _{N \rightarrow \infty} \frac{1}{N} c_{N}=0 \quad \text { almost-surely. }
$$

Turning our attention to $d_{N}$, we note that $\left(N, \alpha_{N} q\right)^{\Uparrow k}$ and $\left(N, c^{\prime} N\right) \Uparrow k$ have the same horizontal coordinates. In particular, there is only one $k$-path travelling between them, and hence $d_{N}$ is identical in distribution to the sum of $k\left(\left\lfloor c^{\prime} N\right\rfloor-\alpha_{N} q\right)$ independent random variables identically distributed like $\beta \omega(0,0)$. In particular, since $\lim _{N \rightarrow \infty} \frac{1}{N} k\left(\left\lfloor c^{\prime} N\right\rfloor-\alpha_{N} q\right)=k\left(c^{\prime}-c\right)$, by applying the law of large numbers we have

$$
\lim _{N \rightarrow \infty} \frac{1}{N} d_{N}=k\left(c^{\prime}-c\right) \beta v \quad \text { almost-surely. }
$$

Finally, by translation, the law of $e_{N}$ is independent of $N$. In particular,

$$
\lim _{N \rightarrow \infty} \frac{1}{N} e_{N}=0 \quad \text { almost-surely. }
$$


By plugging (5.12), (5.13), (5.14), (5.15), and (5.16), into (5.11), we obtain the first equation, (5.8).

Now we turn to proving the second equation, (5.9). Since the proof of (5.9) is similar to the proof of (5.8), we furnish fewer details in this case. Letting $\mathbf{x}$ and $\mathbf{y}$ be nice $k$-points, we assume without loss of generality that $\mathbf{y} \leq 0{ }^{\Uparrow k}$. Moreover, there is a sufficiently small integer $r \in \mathbb{Z}$ such that $x_{r} \leq \mathbf{x}$.

For real numbers $u$, write $\lceil u\rceil$ for the least integer larger than $u$. Now for each integer $N$, define $\gamma_{N}:=\left\lceil c^{\prime} N / q\right\rceil$. Then

$$
\left\lfloor c^{\prime} N\right\rfloor=\gamma_{N} q-\delta_{N},
$$

where $\delta_{N} \in\{0,1, \ldots, q-1\}$. In particular $\left\lfloor c^{\prime} N\right\rfloor \leq \gamma_{N} q$, and moreover $p \gamma_{N}=p\left\lceil c^{\prime} N / q\right\rceil \geq$ $p\lceil c N / q\rceil=p\lceil N / p\rceil \geq N$. Using the definition of $x_{r}$ to obtain the first inequality below, the fact that $\mathbf{y} \leq 0^{\Uparrow k}$ to obtain the third, $p \gamma_{N} \geq N$ in conjunction with Proposition (5.2) to obtain the fourth, and $\left\lfloor c^{\prime} N\right\rfloor \leq \gamma_{N} q$ to obtain the fifth, it follows that

$$
x_{r} \leq \mathbf{x} \leq \mathbf{y}+\left(N, c^{\prime} N\right) \leq\left(N, c^{\prime} N\right)^{\Uparrow k} \leq\left(\gamma_{N} p, c^{\prime} N\right)^{\Uparrow k} \leq x_{\gamma_{N}} .
$$

In particular, by applying the series bound to the chain of $k$-points (5.18) and rearranging, for every $N$ we have the inequality

$$
\frac{1}{N} \log Z_{\mathbf{x} \rightarrow \mathbf{y}+\left(N, c^{\prime} N\right)}(\beta) \leq \frac{1}{N} a_{N}-\frac{1}{N} b-\frac{1}{N} c_{N}-\frac{1}{N} d_{N}-\frac{1}{N} e_{N},
$$

where

$$
\begin{array}{r}
a_{N}:=\log Z_{x_{r} \rightarrow x_{\gamma_{N}}}(\beta), \quad b:=\log Z_{x_{r} \rightarrow \mathbf{x}}(\beta), \quad c_{N}:=\log Z_{y+\left(N, c^{\prime} N\right) \rightarrow(N, c N) \Uparrow k}(\beta), \\
d_{N}:=\log Z_{\left(N, c^{\prime} N\right) \Uparrow k \rightarrow\left(\gamma_{N} p, c^{\prime} N\right) \Uparrow k}(\beta), \quad e_{N}:=\log Z_{\left(\gamma_{N} p, c^{\prime} N\right) \Uparrow k \rightarrow x_{\gamma_{N}}}(\beta) .
\end{array}
$$

Now, in analogy with the proof of equation (5.8), it is straightforward to show that

$$
\lim _{N \rightarrow \infty} \frac{1}{N} b=\lim _{N \rightarrow \infty} \frac{1}{N} c_{N}=\lim _{N \rightarrow \infty} \frac{1}{N} e_{N}=0 \quad \text { almost-surely. }
$$

Now we consider the term $a_{N}$. In the context of Lemma 5.5, we may write $a_{N}:=-p X_{r, \gamma_{N}}$. Using (5.17) to obtain the second equality below, and Lemma 5.5 to obtain the third, we have

$$
\lim _{N \rightarrow \infty} \frac{1}{N} a_{N}:=-\lim _{N \rightarrow \infty} \frac{p}{N} X_{r, \gamma_{N}}=-\frac{c^{\prime}}{c} \lim _{N \rightarrow \infty} \frac{1}{\gamma_{N}} X_{r, \gamma_{N}}=\frac{c^{\prime}}{c} f_{c}(k, \beta) \quad \text { almost-surely. }
$$

Finally, we consider the $d_{N}$ term. Since the vertical coordinates of $\left(N, c^{\prime} N\right)^{\Uparrow k}$ and $\left(\gamma_{N} p, c^{\prime} N\right)^{\Uparrow k}$ are identical, there is only a single $k$-path from $\left(N, c^{\prime} N\right)^{\Uparrow k}$ to $\left(\gamma_{N} p, c^{\prime} N\right)^{\Uparrow k}$, and in particular, $d_{N}$ is simply a sum of $k\left(p \gamma_{N}-N\right)$ independent and identically distributed random variables with the same law as $\beta \omega(0,0)$. Using the fact that $\lim _{N \rightarrow \infty} \frac{1}{N} k\left(p \gamma_{N}-\right.$ $N)=k\left(\frac{c^{\prime}}{c}-1\right)$ and the law of large numbers, we have

$$
\lim _{N \rightarrow \infty} \frac{1}{N} d_{N}=k\left(\frac{c^{\prime}}{c}-1\right) \beta v \quad \text { almost-surely. }
$$

Combining (5.20), (5.21) and (5.22) in (5.19), we obtain (5.9), completing the proof of Lemma 5.6. 
We are now ready to complete the proof of Theorem 2.1 .

Proof of Theorem 2.1 First we recall that in Lemma 5.5, for rational values of $c$ we defined a function $f_{c}(k, \beta)$ as the limit of a rescaled partition function involving diagonal $k$-points at the corners of the $(p, q)$ lattice, where $q / p=c$. We now show that this limit function is continuous in rational values of $c$, and deduce that $f_{c}(k, \beta)$ must also act as a limit function in a broader context.

First of all, if we assume $c^{\prime}$ is also rational, and set $\mathbf{x}=\mathbf{y}=(0,0)^{\Uparrow k}$, it is an immediate consequence of Lemma 5.5 and Lemma 5.6 that for any fixed $k$ and $\beta, F(c):=f_{c}(k, \beta)$ satisfies

$$
F(c)+k v \beta\left(c^{\prime}-c\right) \leq F\left(c^{\prime}\right) \leq \frac{c^{\prime}}{c} F(c)-k \beta v\left(\frac{c^{\prime}}{c}-1\right), \quad \text { for } c<c^{\prime} \text { both rational. }
$$

By rearranging (5.23) we also have

$$
\frac{c}{c^{\prime}} F\left(c^{\prime}\right)+k v \beta\left(1-\frac{c}{c^{\prime}}\right) \leq F(c) \leq F\left(c^{\prime}\right)-k \beta v\left(c^{\prime}-c\right), \quad \text { for } c<c^{\prime} \text { both rational. }
$$

In particular, (5.23) and (5.24) together imply that the function $f_{c}(k, \beta)$, so far only defined for rational values of $c$, is continuous in $c$. In particular, for any $c^{\prime}>0$, we may define

$$
f_{c^{\prime}}(k, \beta):=\lim _{c \uparrow c^{\prime}} f_{c}(k, \beta),
$$

where the limit $c \uparrow c^{\prime}$ in (5.25) is taken through rational values $c$ tending up to $c^{\prime}$.

We are now ready to show that the conclusions of Theorem 2.1 hold for all nice $k$-points $\mathbf{x}$ and $\mathbf{y}$, and for all positive directions $c^{\prime}$, with $f_{c^{\prime}}(k, \beta)$ defined in (5.25) acting as the limit. Indeed, by Lemma 5.6, on the one hand we have

$$
\begin{aligned}
\lim \inf _{N \rightarrow \infty} \frac{1}{N} \log Z_{\mathbf{x} \rightarrow \mathbf{y}+\left(N, c^{\prime} N\right)}(\beta) & \geq \lim _{c \uparrow c^{\prime}}\left\{f_{c}(k, \beta)+k\left(c^{\prime}-c\right) \beta v\right\} \\
& =f_{c^{\prime}}(k, \beta) \quad \text { almost-surely, }
\end{aligned}
$$

and on the other hand

$$
\begin{aligned}
\lim \sup _{N \rightarrow \infty} \frac{1}{N} \log Z_{\mathbf{x} \rightarrow \mathbf{y}+\left(N, c^{\prime} N\right)}(\beta) & \leq \lim _{c \uparrow c^{\prime}}\left\{\frac{c^{\prime}}{c} f_{c}(k, \beta)-k\left(\frac{c^{\prime}}{c}-1\right) \beta v\right\} \\
& =f_{c^{\prime}}(k, \beta) \quad \text { almost-surely. }
\end{aligned}
$$

(Both of the limits taken in (5.26) and (5.27) are taken in rational values $c$ tending up to $c^{\prime}$.) By combining (5.26) and (5.27), we have completed the proof of Theorem 2.1, which states that

$$
\lim _{N \rightarrow \infty} \frac{1}{N} \log Z_{\mathbf{x} \rightarrow \mathbf{y}+\left(N, c^{\prime} N\right)}(\beta)=f_{c^{\prime}}(k, \beta) \quad \text { almost-surely. }
$$

In fact, from our proof of Theorem 2.1, we may immediately deduce Theorem 2.3:

Proof of Theorem 2.3 Since the limit function $f_{c}(k, \beta)$ is continuous in $c$, the inequalities (5.23) and (5.24) hold for all pairs of positive numbers $c<c^{\prime}$. In particular, by setting $k=1$ we prove Theorem 2.3 . 


\subsection{Proof of Theorem 2.2}

We now prove Theorem 2.2, which states that $f_{c}(k, \beta)=k f_{c}(\beta)$, where $f_{c}(\beta):=f_{c}(1, \beta)$. In this section we will use the notation

$$
Z[\mathbf{x} \rightarrow \mathbf{y}]:=Z_{\mathbf{x} \rightarrow \mathbf{y}}(\beta)
$$

since the $k$-points $\mathbf{x}$ and $\mathbf{y}$ appearing in here can be notationally heavy, and we will not be considering different values of $\beta$. The proof of Theorem 2.2 is split into two inequalities, the first of which is significantly easier than the other.

Proof of the Inequality $f_{c}(k, \beta) \leq k f_{c}(\beta)$ Consider setting $\mathbf{x}=(0,0)^{\Uparrow k}=\mathbf{y}$ in Theorem 2.1. Then by definition

$$
\lim _{N \rightarrow \infty} \frac{1}{N} \mathbb{E} \log Z\left[(0,0)^{\Uparrow k} \rightarrow(N, c N)^{\Uparrow k}\right]=f_{c}(k, \beta)
$$

On the other hand, the partition function for the analogous 1-path satisfies

$$
\lim _{N \rightarrow \infty} \frac{1}{N} \mathbb{E} \log Z[(0,0) \rightarrow(N, c N)]=f_{c}(1, \beta)=: f_{c}(\beta) .
$$

However, by the iterated parallel inequality (2.3), for every pair of positive integers $m$ and $n$ we have the bound

$$
Z\left[(0,0)^{\Uparrow k} \rightarrow(n, m)^{\Uparrow k}\right] \leq \prod_{i=1}^{k} Z[(-i+1, i-1) \rightarrow(m-i+1, n+i-1)] .
$$

Since each $Z[(-i+1, i-1) \rightarrow(m-i+1, n+i-1)]$ has the same distribution as $Z[(0,0) \rightarrow(n, m)]$, comparing (5.28) and (5.29) and letting $N \rightarrow \infty$ with $m=c N$ yields the result.

Proof of the Inequality $f_{c}(k, \beta) \geq k f_{c}(\beta)$ Since both $f_{c}(k, \beta)$ and $f_{c}(\beta):=f_{c}(1, \beta)$ are continuous functions of $c$, it suffices to prove the result for rational $c$. To this end, let $p$ and $q$ be positive integers and let $c=q / p$. Then letting $\mathbf{x}=\mathbf{y}=(0,0)^{\Uparrow k}$ in Theorem 2.1 we have

$$
\lim _{M \rightarrow \infty} \frac{1}{M^{2} p} \mathbb{E}\left[\log Z\left[(0,0)^{\Uparrow k} \rightarrow\left(M^{2} p, M^{2} q\right)^{\Uparrow k}\right]=f_{c}(k, \beta) .\right.
$$

The main idea of this proof is to show that the set of $k$-paths from $(0,0)^{\Uparrow k}$ to $\left(M^{2} p, M^{2} q\right)^{\Uparrow k}$ contains a subset of $k$-paths travelling an distance order $M$ apart whose contribution is asymptotically close to $k f_{c}(\beta)$. More specifically, consider the 1-points

$$
z_{i}^{j}(M):=(i-1)(0, M q+1)+j(M p, M q) \quad 1 \leq i \leq k, 0 \leq j \leq M-k,
$$

and for $0 \leq j \leq M-k$ define associated $k$ points $\mathbf{z}^{j}(M):=\left(z_{1}^{j}(M), \ldots, z_{k}^{j}(M)\right)$. It is straightforward to check that

$$
(0,0)^{\Uparrow k} \leq \mathbf{z}^{0}(M) \leq \ldots \leq \mathbf{z}^{j-1}(M) \leq \mathbf{z}^{j}(M) \leq \ldots \leq \mathbf{z}^{M-k}(M) \leq\left(M^{2} p, M^{2} q\right)^{\Uparrow k} .
$$

We remark that since the points $z_{i}^{j}(M)$ form a lattice, the partition functions $Z\left[\mathbf{z}^{j-1}(M)\right.$ $\left.\rightarrow \mathbf{z}^{j}(M)\right]$ are identically distributed for different values of $j$. In particular, by applying the series bound (1.3) to partition functions associated with the chain of $k$-points in (5.30), taking 
logarithms and then expectations, and then using the fact that $Z\left[\mathbf{z}^{j-1}(M) \rightarrow \mathbf{z}^{j}(M)\right]$ are identically distributed, we have

$$
\begin{aligned}
& \frac{1}{M^{2} p} \mathbb{E}\left[\log Z\left[(0,0)^{\Uparrow k} \rightarrow\left(M^{2} p, M^{2} q\right)^{\Uparrow k}\right]\right. \\
& \geq \frac{1}{M^{2} p} \mathbb{E}\left[\log Z\left[(0,0)^{\Uparrow k} \rightarrow \mathbf{z}^{0}(M)\right]\right] \\
& \quad+\frac{1}{M^{2} p} \mathbb{E}\left[\log Z\left[\mathbf{z}^{M-k}(M) \rightarrow\left(M^{2} p, M^{2} q\right)^{\Uparrow k}\right]\right] \\
& \quad+\frac{M-k}{M^{2} p} \mathbb{E}\left[\log Z\left[\mathbf{z}^{0}(M) \rightarrow \mathbf{z}^{1}(M)\right]\right],
\end{aligned}
$$

We now consider the asymptotics of each of the three terms on the right hand side of (5.31). First we look to use Corollary 5.1 to obtain a lower bound for the quantity

$$
\lim \inf _{M \rightarrow \infty} \frac{1}{M^{2} p} \mathbb{E}\left[\log Z\left[(0,0)^{\Uparrow k} \rightarrow \mathbf{z}^{0}(M)\right]\right] .
$$

It is easy to see there is a sufficiently large constant $C_{p, q}$ not depending on $M$ such that $\varpi\left((0,0) \Uparrow k, \mathbf{z}^{0}(M)\right)$, the total number of points contained in any $k$-path from $(0,0) \Uparrow k$ to $\mathbf{z}^{0}(M)$, satisfies

$$
\varpi\left((0,0)^{\Uparrow k}, \mathbf{z}^{0}(M)\right) \leq C_{p, q} M .
$$

Now using Corollary 5.1 to obtain the first inequality below, we have

$$
\mathbb{E}\left[\log Z\left[(0,0)^{\Uparrow k} \rightarrow \mathbf{z}^{0}(M)\right]\right] \geq \varpi\left((0,0)^{\Uparrow k}, \mathbf{z}^{0}(M)\right) \beta v \geq-|\beta v| C_{p, q} M .
$$

In particular,

$$
\lim \inf _{M \rightarrow \infty} \frac{1}{M^{2} p} \mathbb{E}\left[\log Z\left[(0,0)^{\Uparrow k} \rightarrow \mathbf{z}^{0}(M)\right]\right] \geq \lim \inf _{M \rightarrow \infty} \frac{1}{M^{2}}\left(-|\beta \nu| C_{p, q} M\right)=0 .
$$

A similar argument allows us to obtain the bound for the term involving the partition functions of $k$-paths $\mathbf{z}^{M-k}(M)$ to $\left(M^{2} p, M^{2} q\right)^{\Uparrow k}$, giving us

$$
\lim \inf _{M \rightarrow \infty} \frac{1}{M^{2} p} \mathbb{E}\left[\log Z\left[\mathbf{z}^{M-k}(M) \rightarrow\left(M^{2} p, M^{2} q\right)^{\Uparrow k}\right]\right] \geq 0 .
$$

Finally, we now consider the term involving $\mathbb{E}\left[\log Z\left[\mathbf{z}^{0}(M) \rightarrow \mathbf{z}^{1}(M)\right]\right]$. Suppose for each $1 \leq i \leq k$, we have a collection $\pi_{1}, \ldots, \pi_{k}$ of 1-paths such that each $\pi_{i}$ travels from $z_{i}^{0}(M) \rightarrow z_{i}^{1}(M)$. Note that for any $i$, the vertical coordinate of $z_{i+1}^{0}$ is given by $i M q+i$, which is strictly greater that the vertical coordinate of $z_{i}^{1}$, which is given by $i M q+i-1$. This implies that every $k$-tuple of $k$-paths from $\mathbf{z}^{0}(M) \rightarrow \mathbf{z}^{1}(M)$ is guaranteed to be nonintersecting, and hence

$$
Z\left[\mathbf{z}^{0}(M) \rightarrow \mathbf{z}^{1}(M)\right]=\prod_{i=1}^{k} Z\left[z_{i}^{0}(M) \rightarrow z_{i}^{1}(M)\right],
$$

and in particular,

$$
\mathbb{E}\left[\log Z\left[\mathbf{z}^{0}(M) \rightarrow \mathbf{z}^{1}(M)\right]\right]=k \mathbb{E}[\log Z[(0,0) \rightarrow(M p, M q)]] .
$$


Now using (5.32), (5.33) and (5.34) in (5.31), we have

$$
\begin{aligned}
& \lim \inf _{M \rightarrow \infty} \frac{1}{M^{2} p} \mathbb{E}\left[\log Z\left[(0,0)^{\Uparrow k} \rightarrow\left(M^{2} p, M^{2} q\right)^{\Uparrow k}\right]\right. \\
& \geq \lim \inf _{M \rightarrow \infty} \frac{k(M-k)}{M^{2} p} \mathbb{E}[\log Z[(0,0) \rightarrow(M p, M q)]] .
\end{aligned}
$$

Finally, by using the case $k=1$ of Theorem 2.1, we have

$$
\lim _{M \rightarrow \infty} \frac{1}{M p} \mathbb{E}[\log Z[(0,0) \rightarrow(M p, M q)]]=f_{c}(\beta) .
$$

Plugging (5.36) into (5.35), we yield

$$
\lim \inf _{M \rightarrow \infty} \frac{1}{M^{2} p} \mathbb{E}\left[\log Z\left[(0,0)^{\Uparrow k} \rightarrow\left(M^{2} p, M^{2} q\right)^{\Uparrow k}\right] \geq k f_{c}(\beta),\right.
$$

which completes the proof of the inequality $f_{c}(k, \beta) \geq k f_{c}(\beta)$.

\subsection{Proof of Theorem 2.4}

We now prove Theorem 2.4 by a direct application of Kingman's subadditive ergodic theorem, Theorem 5.4.

Proof of Theorem 2.4 Let $h=\left(h^{1}, h^{2}\right)$ such that $h^{1} \leq 0<h^{2}$. For $i \geq 0$, define

$$
x_{i}:=(i-1) h, \quad y_{i}=y+(i-1) h .
$$

For positive integers $r<s$, define the $(s-r)$-points $\mathbf{x}^{r, s}$ and $\mathbf{y}^{r, s}$ by

$$
\mathbf{x}^{r, s}:=\left(x_{r}, x_{r+1}, \ldots, x_{s-1}\right), \quad \mathbf{y}^{r, s}=\left(y_{r}, x_{r+1}, \ldots, y_{s-1}\right),
$$

and define the random variable

$$
X_{r, s}:=\log Z\left[\mathbf{x}^{r, s} \rightarrow \mathbf{y}^{r, s}\right] .
$$

We now verify that with the collection of random variables $\left(X_{r, s}\right)_{0 \leq r<s}$ are in the set up of Theorem 5.4, Kingman's subadditive ergodic theorem. Note first that by the parallel bound (1.6), for any $r<s<t$ we have the subadditivity

$$
X_{r, s}+X_{s, t} \geq X_{r, t},
$$

which proves the first condition. The second condition is immediate since the weights are independent and identically distributed. Finally, the third point followings from Corollary 5.1. To see this, it is simple to verify that $\mathbf{x}^{0, r} \leq \mathbf{y}^{0, r}$ and $\varpi\left(\mathbf{x}^{0, r}, \mathbf{y}^{0, r}\right)=r\|y-x\|_{1}$, and hence by Corollary 5.1

$$
\mathbb{E}\left[X_{0, r}\right]=\mathbb{E}\left[\log Z\left[\mathbf{x}^{0, r} \rightarrow \mathbf{y}^{0, r}\right]\right] \geq \beta \nu\|y-x\|_{1} r .
$$

It follows that we are in the setting of Theorem 5.4, and hence

$$
\frac{1}{k} X_{0, k}=\frac{1}{k} \log Z\left[\mathbf{x}^{h \uparrow k} \rightarrow \mathbf{y}^{h \uparrow k}\right]
$$

converges to a deterministic limit $J_{x, y, h}(\beta)$ depending on $x, y, h$, and $\beta$. Since the weights of the polymer are independent and identically distributed, it is plain that $J$ only depends on $x$ and $y$ through the difference $y-x$. This completes the proof of Theorem 2.4. 
In the sequel we return to writing $Z_{\mathbf{x} \rightarrow \mathbf{y}}(\beta)$ (as opposed to $Z[\mathbf{x} \rightarrow \mathbf{y}]$ ) for the $\mathbf{x}$ to $\mathbf{y}$ partition function.

\subsection{Proof of Theorem 2.5}

To prove Theorem 2.5, we recall Szegö's limit theorem, following Böttcher and Silbermann [5, Chap. 5] and Bump [6, Chap. 42]. Szegö's limit theorem is concerned with the asymptotics of the determinants of Toeplitz matrices-those matrices of the form of the form $\left(d_{j-i}\right)_{1 \leq i, j \leq k}$.

To set up the limit theorem, for every collection of coefficients $\left(d_{m}\right)_{m \in \mathbb{Z}}$ we associate a Laurent series $f(s)=\sum_{m \in \mathbb{Z}} d_{m} s^{m}$ representing a function $f: \mathbb{T} \rightarrow \mathbb{C}$ on the unit circle. This Laurent series is known as the symbol of the Toeplitz matrix.

We define the Wiener and Besov norms of the symbol $f$ by

$$
\|f\|_{W}:=\sum_{m \in \mathbb{Z}}\left|d_{m}\right|<\infty \text { and }\|f\|_{B_{2}^{1 / 2}}:=\sum_{m \in \mathbb{Z}}(|m|+1)\left|d_{m}\right|^{2}<\infty
$$

respectively. We say $f \in W \cap B_{2}^{1 / 2}$ if both $\|f\|_{W}$ and $\|f\|_{B_{2}^{1 / 2}}$ are finite. Recalling the definition of winding number stated in (2.11), we now state Szegö's limit theorem.

Theorem 5.7 (Szegö's limit theorem) Suppose $f(s):=\sum_{m \in \mathbb{Z}} d_{m} s^{m}$ is a Laurent series in $W \cap B_{2}^{1 / 2}$ with no zeroes on $\mathbb{T}$ and winding number zero. Moreover, suppose there is a Laurent series $\sum_{m \in \mathbb{Z}} c_{m} s^{m}$ satisfying

$$
f(s)=\exp \left(\sum_{m \in \mathbb{Z}} c_{m} s^{m}\right) .
$$

Then

$$
\lim _{k \rightarrow \infty}\left(e^{-k c_{0}} \operatorname{det}_{i, j=1}^{k}\left(d_{j-i}\right)\right)=\exp \left(\sum_{m=1}^{\infty} m c_{m} c_{-m}\right) .
$$

We now use the Lindström-Gessel-Viennot formula to relate the asymptotics of manypath infinite temperature polymer to the asymptotics of Toeplitz determinants. Now, for $k$-points $\mathbf{x}$ and $\mathbf{y}$, by the (2.4)

$$
Z_{\mathbf{x} \rightarrow \mathbf{y}}(0)=\operatorname{det}_{i, j=1}^{k}\left(Z_{x_{i} \rightarrow y_{j}}(0)\right) .
$$

We are interested in circumstances where the matrix $\left(Z_{x_{i} \rightarrow y_{j}}(0)\right)_{1 \leq i, j \leq k}$ is a Toeplitz matrix for every $k$, which occurs whenever each $Z_{x_{i} \rightarrow y_{j}}(0)$ is a function of $j-i$ only, and the $k$-points $\mathbf{x}$ and $\mathbf{y}$ have the form

$$
\mathbf{x}_{i}=x+(i-1) h, \quad \mathbf{y}_{i}=y+(i-1) h, \quad i=1, \ldots, k .
$$

for some base points $x$ and $y$ in $\mathbb{Z}^{2}$, and some direction $h \in \mathbb{Z}^{2}$. That is, $\mathbf{x}$ and $\mathbf{y}$ are of the form $\mathbf{x}^{h \uparrow k}$ and $\mathbf{y}^{h \uparrow k}$.

Indeed, when $\mathbf{x}=\mathbf{x}^{h \uparrow k}$ and $\mathbf{y}=\mathbf{y}^{h \uparrow k}$, each $Z_{x_{i} \rightarrow y_{j}}(0)=d_{j-i}$, where

$$
d_{m}:=\left(\begin{array}{c}
z^{1}+z^{2}+m\left(h^{1}+h^{2}\right) \\
z^{1}+m h^{1}
\end{array}\right),
$$


where $z:=y-x$, and it follows that the partition function may be written as a Toeplitz determinant: $Z_{\mathbf{x} \rightarrow \mathbf{y}}(0)=\operatorname{det}_{i, j=1}^{k}\left(d_{j-i}\right)$. Now define the symbol

$$
a_{z, h}(s):=\sum_{m \in \mathbb{Z}} d_{m} s^{m} .
$$

Whenever $h_{1}<0<h_{2}$, it is clear that only finitely many of the $d_{m}$ are non-zero, and hence $a_{z, h}(s):=\sum_{m \in \mathbb{Z}} d_{m} s^{m}$ is contained in $W \cap B_{S}^{1 / 2}$. By the assumption in Theorem 2.5, $a_{z, h}$ has winding number 0, and it follows that we are in the set up of the Szegö's limit theorem, Theorem 5.7. In particular, if $\left(c_{m}\right)_{m \in \mathbb{Z}}$ are such that

$$
a_{z, h}(s)=\exp \left(\sum_{m \in \mathbb{Z}} c_{m} s^{m}\right)
$$

then

$$
\lim _{k \rightarrow \infty}\left(e^{-k c_{0}} Z_{\mathbf{x}^{h \uparrow k} \rightarrow \mathbf{y}}{ }^{h \uparrow k}(0)\right)=\lim _{k \rightarrow \infty}\left(e^{-k c_{0}} \underset{i, j=1}{k} \operatorname{det}_{i, j}\left(d_{j-i}\right)\right)=\exp \left(\sum_{m=1}^{\infty} m c_{m} c_{-m}\right),
$$

completing the proof of Theorem 2.5.

\subsection{The Asymptotics of the High-Temperature Scaled-k Limit}

This section is devoted to proving Lemma 2.6. In this direction, first we state-and, for completeness, include a proof of - the following result regarding the infinite temperature partition function on a finite rectangle. We note that, via the well-known bijection between non-intersecting paths and plane partitions, this is equivalent to Macmahon's formula. We refer the reader to Stanley [42, Sect. 7.20] for an approach using the RSK correspondence.

Proposition 5.8 Let $m, n, k$ be positive integers satisfying $k \leq m \wedge n$. Then

$$
Z_{(1,1)^{\uparrow k} \rightarrow(n, m) \downarrow k}(0)=\frac{H(m+n-k) H(k) H(m-k) H(n-k)}{H(m) H(n) H(m+n-2 k)},
$$

where $H(N):=\prod_{j=0}^{N-1} j !$ is the superfactorial.

Proof Let $m, n, k$ be positive integers satisfying $k \leq m \wedge n$, and suppose $\pi=\left(\pi_{1}, \ldots, \pi_{k}\right)$ is a $k$-path going from $(1,1)^{\uparrow k} \rightarrow(n, m)^{\downarrow k}$. It is easily seen that for each $i$, the $i^{\text {th }}$ path $\pi_{i}$ must go through the points $x_{i}=(k+1-i, i)$ and $y_{i}:=(n-k+i, m-i+1)$. Let $\mathbf{x}=\left(x_{1}, \ldots, x_{k}\right)$ and $y=\left(y_{1}, \ldots, y_{k}\right)$ be the associated $k$-points. Then we have the following identity for the rectangular zero-temperature partition function

$$
Z_{(1,1) \uparrow k} \rightarrow(n, m) \downarrow k(0)=Z_{\mathbf{x} \rightarrow \mathbf{y}}(0) .
$$

By the Lindström-Gessel-Viennot formula (2.4),

$$
Z_{\mathbf{x} \rightarrow \mathbf{y}}(0)=\operatorname{det}_{i, j=1}^{k}\left(Z_{x_{i} \rightarrow y_{j}}(0)\right) .
$$

Now note that the partition function $Z_{x_{i} \rightarrow y_{j}}(0)$ simply counts the number of paths from $x_{i}$ to $y_{j}$, that is

$$
Z_{x_{i} \rightarrow y_{j}}(0)=\left(\begin{array}{c}
m+n-2 k \\
m-k+j-i
\end{array}\right)
$$


It bares remarking at this stage that the right-hand-side of (5.41) can be expanded as a more tractable determinant than the left-hand-side, since the path length from each $x_{i}$ to each $y_{i}$ is $m+n-2 k$, which does not depend on $i$ or $j$.

According to a well known determinant identity (see Krattenthaler [27, Equation (2.17)]),

$$
\operatorname{det}_{i, j=1}^{k}\left(\left(\begin{array}{c}
a+b \\
a+j-i
\end{array}\right)\right)=\prod_{r=1}^{k} \prod_{s=1}^{a} \prod_{t=1}^{b} \frac{r+s+t-1}{r+s+t-2} .
$$

Moreover, it easily verified that

$$
\prod_{r=1}^{k} \prod_{s=1}^{a} \prod_{t=1}^{b} \frac{r+s+t-1}{r+s+t-2}=\frac{H(k+a+b) H(k) H(a) H(b)}{H(k+a) H(k+b) H(a+b)},
$$

where $H(N):=\prod_{j=1}^{N-1} j$ ! is the superfactorial. Setting $a=m-k$ and $b=n-k$, and combining (5.41), (5.42), (5.43) and (5.44), we obtain (5.40).

Proof of Lemma 2.6 To prove Lemma 2.6, it remains to study the large- $N$ asymptotics of (5.40) when $m=c N, n=N$ and $k=\alpha N$, for any $c>0$ and $\alpha \leq c \wedge 1$. By adapting (4.26), we see that for $p>0$,

$$
\log H(p N)=N^{2}\left(\frac{p^{2}}{2} \log p+\frac{p^{2}}{2} \log N-\frac{3 p^{2}}{4}+o(1)\right) .
$$

Now the result follows from (5.40) and (5.45), noting the identity

$$
(c+1-\alpha)^{2}+\alpha^{2}+(c-\alpha)^{2}+(1-\alpha)^{2}-c^{2}-1^{2}-(c+1-2 \alpha)^{2}=0 .
$$

\section{Proofs of Results in Section 3}

\subsection{Proofs of Proposition 3.2}

In this section we prove Propositions 3.2, which states that certain random functions associated with the partition functions of a log-gamma polymer may be expressed in terms of a stochastic interface. Proposition 3.2 follows in essence from the main result in Corwin et al. [14], though the explicit formulation we use is closer to the set up O'Connell et al. [37], and hence we choose to follow the exposition of the latter. We will be following Sects. 2 and 3 of [37], though in order to maintain consistency in our notation, we will index our variables differently here to [37]. In particular, in the present paper we work in the more restrictive square case, and hence the quantities $h, n$ and $m$ appearing in [37] will all be equal to $N$ here.

We now introduce the geometric RSK correspondence, a bijective mapping

$$
T: \mathbb{R}_{>0}^{N \times N} \rightarrow \mathbb{R}_{>0}^{N \times N}
$$

O'Connell et al. [37] give a definition of the geometric RSK correspondence first via a sequence of 'local moves', and then in an alternative expression in terms of non-intersecting lattice paths. Focussing here only on the latter approach, for $m \geq k$ define the partition functions

$$
\tau_{m, k}(w):=\sum_{\pi \in \Gamma^{N}(m, k)} \prod_{(i, j) \in \pi} w_{i, j}
$$


and

$$
\tilde{\tau}_{m, k}(w):=\sum_{\pi \in \Gamma^{N}(m, k)} \prod_{(i, j) \in \pi} w_{j, i} .
$$

Now given a set of weights $w:=\left(w_{i, j}: 1 \leq i, j \leq N\right)$, we define $\left(t_{i, j}\right):=T\left(w_{i, j}\right)$ by setting

$$
t_{i, j}:=\left\{\begin{array}{l}
\tau_{N-j+i, i}(w) / \tau_{N-j+i, i-1}(w), \quad 1 \leq i \leq j \leq N, \\
\tilde{\tau}_{N-i+j, j}(w) / \tilde{\tau}_{N-i+j, j-1}(w), \quad 1 \leq j \leq i \leq N .
\end{array}\right.
$$

(The Eq. (6.1) amounts to [37, (3.10)].)

Now, given a function $f: S_{N} \rightarrow \mathbb{R}$, we define the diagonal products $\rho_{0}, \ldots, \rho_{N}$ and $\tilde{\rho}_{0}, \ldots, \tilde{\rho}_{N}$ of $f$ by first setting

$$
d_{k}:=\prod_{(i, j) \in S_{N}: j-i=k} f(i, j) \text { for } k \in \mathbb{Z}
$$

(with the convention that the empty product is equal to 1 ) and now letting

$$
\rho_{k}:=d_{N-k} \quad \tilde{\rho}_{k}:=d_{k-N} .
$$

Finally, define

$$
f^{\alpha, \hat{\alpha}}:=\prod_{k=1}^{N}\left(\rho_{k} / \rho_{k-1}\right)^{\alpha_{k}} \prod_{k=1}^{N}\left(\tilde{\rho}_{k} / \tilde{\rho}_{k-1}\right)^{\hat{\alpha}_{k}} .
$$

The definition for $f^{\alpha, \hat{\alpha}}$ is based on Eq. (2.9) of [37]. Finally, turning to the definition (3.12) of [37], given a function $f: S_{N} \rightarrow \mathbb{R}$, for $s \in \mathbb{C}$ define

$$
\mathcal{E}_{S}(f):=\frac{s}{f(N, N)}+\sum_{\langle x, y\rangle \in S_{N}^{*}} \frac{f(y)}{f(x)} .
$$

We are now ready to use Corollary 3.3 of [37], which states that if the weights $w:=\left(w_{i, j}\right.$ : $1 \leq i, j \leq N)$ are random and distributed according to a certain probability distribution, then the law of the random variable $t=T(w)$ has a tractable representation. More specifically, define the probability measure

$$
\mathcal{I}_{\theta, \hat{\theta} ; s}(d w):=\prod_{i, j} \Gamma\left(\hat{\theta}_{i}+\theta_{j}\right)^{-1} \exp \left(-\sum_{1 \leq i, j \leq N} 1 / w_{i, j}\right) \prod_{i, j} w_{i, j}^{-\hat{\theta}_{i}-\theta_{j}} d w_{i, j} / w_{i, j},
$$

where we require that each $\hat{\theta}_{i}+\theta_{j}>0$. Namely, $\mathcal{I}_{\theta, \hat{\theta}}$ is the product measure of a collection of independent inverse-gamma distributed random variables with parameters $\hat{\theta}_{i}+\theta_{j}$. We are now ready to give the statement.

Lemma 6.1 (Corollary 3.3 of [37]) If $\left(w_{i, j}\right)$ are distributed according to $\mathcal{I}_{\theta, \hat{\theta}}$, then the random function $t: S_{N} \rightarrow \mathbb{R}_{>0}$ defined by setting $t:=T(w)$ is distributed according to the probability measure

$$
\prod_{1 \leq i, j \leq N} \Gamma\left(\hat{\theta}_{i}+\theta_{j}\right)^{-1} t^{-\theta,-\hat{\theta}} e^{-\mathcal{E}_{1}(t)} \prod_{1 \leq i, j \leq N} \frac{d t_{i, j}}{t_{i, j}} .
$$

We are now equipped to prove Proposition 3.2. 
Proof of Proposition 3.2 Consider setting $\theta_{j}=\mu$ and $\hat{\theta}_{i}=0$ for each $i, j$ in Lemma 6.1. Namely, this reduces to the statement that if $w:=\left(w_{i, j}: 1 \leq i, j \leq N\right)$ are independent and identically distributed with the inverse gamma law of parameter $\mu$, then the random function $t: S_{N} \rightarrow \mathbb{R}_{>0}$ defined by setting $t=T(w)$ is distributed according to the probability measure

$$
\Gamma(\mu)^{-N^{2}} \prod_{i=1}^{N} t(i, i)^{-\mu} e^{-\mathcal{E}_{1}(t)} \prod_{1 \leq i, j \leq N} \frac{d t_{i, j}}{t_{i, j}} .
$$

Now define the random function $\varphi_{\mu}^{N}: S_{N} \rightarrow \mathbb{R}$ by setting $\varphi_{\mu}^{N}(i, j):=\log t_{i, j}$. First we remark that by (6.1), $\varphi_{\mu}^{N}$ agrees with the definition given in (3.9). Secondly, it is plain by transforming the density (6.2) that the law of $\varphi_{\mu}^{N}(i, j)$ is given by

$$
\Gamma(\mu)^{-N^{2}} \exp \left(-e^{-\phi(N, N)}-\mu \sum_{i=1}^{N} \phi(i, i)-\sum_{\langle x, y\rangle \in S_{N}^{*}} e^{\phi(y)-\phi(x)}\right) \prod_{z \in S_{N}} d \phi(z) .
$$

In other words, $\varphi_{\mu}^{N}$ has the same distribution as the stochastic interface defined in Proposition 3.2, completing the proof.

\subsection{Proof of Proposition 3.4}

In this section we prove Proposition 3.4, which states that the eigenvalue processes of certain random matrices may be expressed in terms of stochastic interfaces. In the proof of Proposition 3.4, we will require the following result by Baryshnikov, which we paraphrase in terms of the eigenvalue process interface $\varphi_{H, U}$ defined in (3.12).

Theorem 6.2 (Baryshnikov [3], Proposition 4.7) Let $H$ be a unitarily invariant $N \times N$ random matrix. Then conditional on the diagonal values $\varphi_{H, U}(1,1)=\lambda_{1}, \ldots, \varphi_{H, U}(N, N)=\lambda_{N}$, the law of $\varphi_{H, U}$ on the triangle $T_{N}$ is uniformly distributed on the set of Gelfand-Tsetlin pattern with diagonal $\lambda_{1}, \ldots, \lambda_{N}$.

Proof of Proposition 3.4 We prove the result for the eigenvalue process associated with the Gaussian unitary ensemble, omitting a proof for the Laguerre unitary ensemble, which is almost identical.

Let $\varphi^{N}: S_{N} \rightarrow \mathbb{R}$ be a stochastic interface with interaction potential bead and weight functions $W_{i, j}^{\mathrm{GUE}}(u)=\mathbb{1}_{i=j} \frac{1}{2} u^{2}$. By Lemma 3.1 and the continuous Gelfand-Tsetlin volume formula (3.3), it is plain that the diagonal $\left(\lambda_{1}, \ldots, \lambda_{N}\right):=\left(\varphi^{N}(1,1), \ldots, \varphi^{N}(N, N)\right)$ is distributed according to the probability measure

$$
Z_{N}^{-1} \Delta_{N}(\lambda)^{2} \exp \left(-\frac{1}{2} \sum_{i=1}^{N} \lambda_{i}^{2}\right) \prod_{i=1}^{N} d \lambda_{i}
$$

where $Z_{N}$ is some normalisation constant. It is well known that the $N$ eigenvalues $\lambda_{1}>$ $\ldots>\lambda_{N}$ of a matrix $H$ distributed according to the Gaussian Unitary Ensemble also have this law (see e.g. Mehta [31, Theorem 3.3.1]). In other words, the stochastic interfaces $\varphi^{N}$ and $\varphi_{H, U}^{N}$ have the same marginal distribution on the diagonal $i=j$.

It is now a consequence of Proposition 6.2 that the equality in distribution for $\varphi_{H, U}^{N}$ and $\varphi^{N}$ holds not just on the diagonal but on $T_{N}$. Likewise, by the distribution symmetry of both 
the eigenvalue process $\varphi_{H, U}$ and the stochastic interface $\varphi$ about the diagonal $i=j, \varphi_{H, U}$ and $\varphi$ have the same marginal law on the reflected triangle $\tilde{T}_{N}:=\{(i, j): 1 \leq j \leq i \leq N\}$. It remains to establish that the processes $\left\{\varphi_{H, U}(x): x \in T_{N}\right\}$ and $\left\{\varphi_{H, U}: y \in \tilde{T}_{N}\right\}$ are conditionally independent given the diagonal $\left\{\varphi_{H, U}(i, i): 1 \leq i \leq N\right\}$, but this holds immediately from the fact that $U$ is independent of $H$. It follows that $\varphi_{H, U}$ has the same law as $\varphi$ on the entire square $S_{N}$, completing the proof in the GUE case.

\subsection{Proof of Theorem 3.5}

Proof of Theorem 3.5 The rescaled process $\theta_{\mu}^{N}:=\mu \varphi_{\mu}^{N}$ has law $\tilde{P}_{\mu}^{N}(d \theta):=(\mu \Gamma(\mu))^{-N^{2}}$ $f_{\mu}(\theta) d \theta$, where

$$
f_{\mu}(\theta):=\exp \left(-\sum_{\langle x, y\rangle \in S_{N}^{*}} e^{\mu^{-1}(\theta(y)-\theta(x))}-\sum_{i=1}^{N} \theta(i, i)-e^{-\mu^{-1} \theta(N, N)}\right) .
$$

We note that as $\mu \downarrow 0, f_{\mu}(\theta)$ converges pointwise almost-everywhere on $\mathbb{R}^{S_{N}}$ to $f_{0}(\theta)$, where

$$
f_{0}(\theta):=\mathbb{1}_{\theta(N, N) \geq 0} \prod_{\langle x, y\rangle \in S_{N}^{*}} \mathbb{1}_{\theta(y) \leq \theta(x)} \exp \left(-\sum_{i=1}^{N} \theta(i, i)\right) .
$$

Fix $\ell \in \mathbb{R}$ and let $\left\{A_{z}: z \in S_{N}\right\}$ be a collection of compact subsets of $[\ell, \infty)$. Consider a cylinder subset of $\mathbb{R}^{S_{N}}$ of the form

$$
A:=\left\{\theta: S_{N} \rightarrow \mathbb{R} \text { satisfying } \theta(z) \in A_{z} \text { for each } z \in S_{N}\right\} .
$$

As $\mu$ varies, the functions $f_{\mu}(\theta) \mathbb{1}_{A}(\theta)$ are uniformly bounded by $e^{-N \ell} \mathbb{1}_{A}(\theta)$, which is integrable since each $A_{z}$ is compact. It follows that by the bounded convergence theorem that $\int_{\mathbb{R}^{S_{N}}} f_{\mu}(\theta) \mathbb{1}_{A}(\theta) d \theta \rightarrow \int_{\mathbb{R}^{S_{N}}} f_{0}(\theta) \mathbb{1}_{A}(\theta) d \theta$. Using the fact that $\mu \Gamma(\mu) \rightarrow 1$ as $\mu \downarrow 0$, we see that as $\mu \downarrow 0$,

$$
\tilde{P}_{\mu}^{N}(A) \rightarrow \tilde{P}_{0}^{N}(A)
$$

where $\tilde{P}_{0}^{N}(d \theta)=f_{0}(\theta) d \theta$, which is precisely the law of the LUE eigenvalue process. Since $\ell$ and the $A_{z}$ were arbitrary, this proves the result.

\subsection{Proof of Theorem 3.7}

In this section we consider the large- $\mu$ asymptotics of the interface $\varphi_{\mu}^{N}$, providing a proof of Theorem 3.7.

Proof of Theorem 3.7 By definition, the law of the interface $\varphi_{\mu}^{N}: S_{N} \rightarrow \mathbb{R}$ is proportional to

$$
\exp \left(-e^{-\phi(N, N)}-\mu \sum_{i=1}^{N} \phi(i, i)-\sum_{\langle x, y\rangle \in S_{N}^{*}} e^{\phi(y)-\phi(x)}\right) .
$$

We want to take a change of variables so that the interaction term competes with the weight term for large $\mu$. To this end, consider the change of variables

$$
\theta_{\mu}^{N}(i, j):=\varphi_{\mu}^{N}(i, j)+(2 N+1-j-i) \log \mu .
$$


It is straightforward to show that the random function $\theta_{\mu}^{N}: S_{N} \rightarrow \mathbb{R}$ is itself a stochastic interface whose law is proportional to $\exp \left(-\mu \mathcal{F}^{N}[\theta]\right)$, where

$$
\mathcal{F}^{N}[\phi]:=e^{-\phi(N, N)}+\sum_{i=1}^{N} \phi(i, i)+\sum_{\langle x, y\rangle \in S_{N}^{*}} e^{\phi(y)-\phi(x)} .
$$

Plainly, as $\mu \rightarrow \infty$ the interface $\theta_{\mu}^{N}$ converges in distribution to the deterministic function $\theta_{\min }^{N}: S_{N} \rightarrow \mathbb{R}$ minimising $F^{N}[\theta]$. That completes the proof that $\theta_{\mu}^{N}$ converges in distribution to a deterministic function $\theta_{\mathrm{min}}^{N}$ minimising a variational problem. It remains to show that this minimiser has the form (3.19).

Since the law of $\theta_{\mu}^{N}$ is symmetric about the diagonal $i=j$, the limit function $\theta_{\min }$ must also be symmetric, and without loss of generality we consider $\theta_{\min }(i, j)$ for $i \leq j$.

To compute $\theta_{\min }(i, j)$, we consider the direct implications for the polymer partition functions of sending $\mu \rightarrow \infty$. For integer values of $\mu, \frac{1}{\zeta_{\mu}(z)}$ can be written as a sum of $\mu$ independent and identically distributed exponential variables with mean 1 . It follows that as $\mu \rightarrow \infty$

$$
\frac{1}{\mu \zeta_{\mu}(z)} \text { converges in distribution to } 1 \text {. }
$$

Since each product in the sum $\tau_{\mu}^{N}(m, k):=\sum_{\pi \in \Gamma^{N}(m, k)} \prod_{z \in \pi} \zeta_{\mu}(z)$ contains $k(N+m-k)$ weights, we have

$$
\mu^{k(N+m-k)} \tau_{\mu}^{N}(m, k) \text { converges in distribution to \# } \Gamma^{N}(m, k) .
$$

Now using the definitions of $\varphi_{\mu}^{N}$ and $\theta_{\mu}^{N}$ (which appear in (3.9) and (6.4) respectively), for $i \leq j$ we have

$$
\begin{aligned}
\theta_{\mu}^{N}(i, j) & :=\varphi_{\mu}^{N}(i, j)+(2 N+1-(j+i)) \log \mu \\
& :=\log \left(\frac{\mu^{i(N+(N-j+i)-i)} \tau_{\mu}^{N}(N-j+i, i)}{\mu^{(i-1)(N+(N-j+i)-(i-1))} \tau_{\mu}^{N}(N-j+i, i-1)}\right) .
\end{aligned}
$$

Combining (6.8) with (6.7), it follows that as $\mu \rightarrow \infty$

$$
\theta_{\mu}^{N}(i, j) \text { converges in distribution to } \log \left(\frac{\# \Gamma^{N}(N-j+i, i)}{\# \Gamma^{N}(N-j+i, i-1)}\right) .
$$

It remains to compute

$$
\theta_{\min }^{N}(i, j)=\log \left(\frac{\# \Gamma^{N}(N-j+i, i)}{\# \Gamma^{N}(N-j+i, i-1)}\right) .
$$

In Lemma 5.8, we gave the following explicit expression for the cardinality of each $\Gamma^{N}(m, k)$

$$
\# \Gamma^{N}(m, k)=\frac{H(N+m-k) H(k) H(N-k) H(m-k)}{H(N) H(m) H(N+m-2 k)} .
$$

Combining (6.10) with (6.11), and using the definition $H(n):=\prod_{i=1}^{N}(i-1)$ ! of the superfactorial, after some calculation we obtain the formula

$$
\theta_{\min }^{N}(i, j):=\log \left(\frac{(i-1) !(2 N-j-i+1) !(2 N-j-i) !}{(2 N-j) !(N-j) !(N-i) !}\right), \quad i \leq j,
$$

completing the proof. 


\section{Proofs of Results in Sect. 4}

\subsection{Proof of Theorem 4.6}

In this section we prove Theorem 4.6.

Proof of Theorem 4.6 Since $\theta_{\mathrm{min}}^{N}$ is symmetric, the limit function of the rescaled interface $\bar{\theta}_{\text {min }}^{N}$ must also be symmetric, and hereafter without loss of generality we consider $\{s \leq t\}$. In this case, by (3.19) and Definition 4.3, the rescaled interface associated with $\theta_{\min }^{N}$ is given by

$$
\bar{\theta}_{\min }^{N}(s, t)=\frac{1}{N} \log \left(\frac{(i-1) !(2 N-j-i+1) !(2 N-j-i) !}{(2 N-j) !(N-j) !(N-i) !}\right) \quad \text { for } i=\lfloor s N\rfloor \text { and } j=\lfloor t N\rfloor .
$$

By Stirling's formula, $\frac{1}{N} \log (\lfloor u N\rfloor)=u(\log N-1)+q(u)+o(1)$, where $q(u):=u \log u$. Using the fact that

$$
s+2(2-s-t)-(2-t)-(1-t)-(1-s)=0,
$$

it follows that $\bar{\theta}_{\mathrm{min}}^{N}(s, t) \rightarrow \xi_{\mathrm{ht}}(s, t)$, given by

$$
\xi_{\text {ht }}(s, t)=q(s)+2 q(2-s-t)-q(2-t)-q(1-t)-q(1-s) .
$$

\subsection{Calculations Surrounding Conjecture 4.7}

Section 7.2 is dedicated to sketching calculations leading us to Conjecture 4.7, which anticipaties Gaussian fluctuations of the logarithmic partition functions of the log-gamma polymer at high temperature.

First of all, consider that by the central limit theorem, for each $z$, as $\mu \rightarrow \infty$ the random variable

$$
N_{\mu}(z):=\frac{1 / \zeta_{\mu}(z)-\mu}{\sqrt{\mu}}
$$

converges in distribution to a standard normal random variable. Now define the variable $r_{\mu}(z)$ through the equation

$$
1+\mu^{-1 / 2} r_{\mu}(z)=\mu \zeta_{\mu}(z)
$$

It is straightforward to check that $r_{\mu}(z)=\left(1+\mu^{-1 / 2} N_{\mu}(z)\right)^{-1} N_{\mu}(z)$, and hence $r_{\mu}(z)$ also converges in distribution to a standard normal random variable as $\mu \rightarrow \infty$.

Assuming (4.18) and (4.19), we now consider the asymptotics of the partition functions under a suitable scaling as $\mu$ and $N$ tend to infinity. Consider the random variable

$$
G_{\mu}^{N}(m, k):=\frac{\mu^{k(N+m-k)} \tau_{\mu}^{N}(m, k)}{\# \Gamma^{N}(m, k)}
$$


Using (7.1) and the fact that each path in $\Gamma^{N}(m, k)$ contains $k(N+m-k)$ weights, we have

$$
\begin{aligned}
G_{\mu}^{N}(m, k) & =\frac{1}{\# \Gamma^{N}(m, k)} \sum_{\pi \in \Gamma^{N}(m, k)} \prod_{z \in \pi}\left(1+\frac{1}{\sqrt{\mu}} r_{\mu}(z)\right) \\
& =\frac{1}{\# \Gamma^{N}(m, k)} \sum_{\pi \in \Gamma^{N}(m, k)} \sum_{F \subset \pi} \prod_{z \in F} \frac{1}{\sqrt{\mu}} r_{\mu}(z) \\
& =\sum_{F \subset S_{N}} \mu^{-\# F / 2} Q_{m, k}^{N}(F \in \pi) \prod_{z \in F} r_{\mu}(z),
\end{aligned}
$$

where the sum in the final line above is taken over all subsets $F$ of the square $S_{N}:=$ $\{1, \ldots, N\}^{2}$. Expanding further, and considering the $n$ ! ways of ordering the elements of a set $F$ of size $n$, we have

$$
G_{\mu}^{N}(m, k)=\sum_{n=0}^{\infty} \frac{\mu^{-n / 2}}{n !} \sum_{\left(z_{1}, \ldots, z_{n}\right) \in S_{N}^{(n)}} Q_{m, k}^{N}\left(\left\{z_{1}, \ldots, z_{n}\right\} \in \pi\right) \prod_{i=1}^{n} r_{\mu}\left(z_{i}\right),
$$

where the internal sum is taken over all distinct $n$-tuples of elements of $S_{N}$. When $N$ is large, for each $n$ the set of non-distinct $k$-tuples $S_{N}^{n}-S_{N}^{(n)}$ is small compared to the set of distinct $k$-tuples $S_{N}^{(n)}$. Using this fact as well as the asymptotic decoupling (4.19), we anticipate that when $N$ is large, and both $m$ and $k$ have order $N$, then

$$
\sum_{\left(z_{1}, \ldots, z_{n}\right) \in S_{N}^{(n)}} Q_{m, k}^{N}\left(\left\{z_{1}, \ldots, z_{n}\right\} \in \pi\right) \prod_{i=1}^{n} r_{\mu}\left(z_{i}\right)=(1+o(1))\left(\sum_{z \in S_{N}} Q_{m, k}^{N}(z \in \pi) r_{\mu}(z)\right)^{n}
$$

in which case using (7.2) reduces to

$$
G_{\mu}^{N}(m, k)=(1+o(1)) \sum_{n=0}^{\infty} \frac{1}{n !}\left(\mu^{-1 / 2} \sum_{z \in S_{N}} Q_{m, k}^{N}(z \in \pi) r_{\mu}(z)\right)^{n} .
$$

Taking the scaling $\mu=\kappa N^{2}$, and using (7.3), we anticipate that

$$
\log G_{\kappa N^{2}}^{N}(c N, \alpha N) \rightarrow \frac{1}{\kappa} \int_{S} q_{c, \alpha}(u, v) \dot{W}(u, v),
$$

where $q_{c, \alpha}(u, v)$ is defined in (4.18), and $\dot{W}$ is a space-time white noise on $S$ independent of $c$ and $\alpha$. (We remark that by considering $\tilde{\tau}$ in place of $\tau$, we also expect

$$
\log \tilde{G}_{\kappa N^{2}}^{N}(c N, \alpha N):=\log \left(\frac{\mu^{k(N+m-k)} \tilde{\tau}_{\mu}^{N}(m, k)}{\# \tilde{\Gamma}^{N}(m, k)}\right)
$$

converges in distribution to $\frac{1}{\kappa} \int_{S} \tilde{q}_{c, \alpha}(u, v) \dot{W}(u, v)$, where $\tilde{q}$ is defined in analogy to (4.18), instead using the uniform measure $\tilde{Q}_{m, k}^{N}$ on $\tilde{\Gamma}^{N}(m, k)$, the set of $k$-paths travelling from $(1,1)^{\uparrow k}$ to $(m, N)^{\downarrow k}$.)

Now define

$$
H_{\kappa}^{N}(s, t):= \begin{cases}\log G_{\kappa N^{2}}^{N}(t N, s N) & s \leq t \\ \log \tilde{G}_{\kappa N^{2}}^{N}(s N, t N) & s \geq t\end{cases}
$$


Then by (7.4), we anticipate that $H_{\kappa}^{N}$ converges in distribution to a Gaussian process $H_{\kappa}(s, t)$ on $S$ with convariance

$$
\mathbb{E}\left[H_{\kappa}(s, t) H_{\kappa}\left(s^{\prime}, t^{\prime}\right)\right]=\frac{1}{\kappa^{2}}\left\langle h_{s, t}, h_{s^{\prime}, t^{\prime}}\right\rangle_{L^{2}(S)}
$$

where for $s \leq t, h_{s, t}(u, v):=q_{t, s}(u, v), h_{t, s}(u, v):=h_{s, t}(v, u)$, and $\langle f, g\rangle_{L^{2}(S)}:=$ $\int_{S} f(z) g(z) d z$.

\section{Derivation of the Surface Tension of the Bead Model}

\subsection{Coordinate Changes and Wulff Functionals}

It will be useful to define the change of coordinates mapping the set $T:=\{(x, y): 0 \leq x \leq$ $y \leq 1\}$ to set $U:=\{(r, \tau): 0 \leq \tau \leq 1, \tau / 2 \leq r \leq 1-\tau / 2\}$ defined by setting

$$
\tau:=y-x \text { and } r:=\frac{y+x}{2} .
$$

We say that $(r, \tau)$ are the tilted coordinates. Note that the transformation $(x, y) \mapsto(r, \tau)$ has unit Jacobian, and for suitable functions $f$ we have

$$
\frac{\partial f}{\partial x}=\frac{1}{2} \frac{\partial f}{\partial r}-\frac{\partial f}{\partial \tau} \quad \text { and } \quad \frac{\partial f}{\partial y}=\frac{1}{2} \frac{\partial f}{\partial r}+\frac{\partial f}{\partial \tau} .
$$

It turns out to be useful to study the surface tension in the tilted change of coordinates $(r, \tau)$. In light of (8.2), we define the surface tension associated with an interaction potential $V$ in the tilted coordinates by

$$
\sigma_{\text {tilted }}^{V}(p, q):=\sigma^{V}\left(\frac{1}{2} p-q, \frac{1}{2} p+q\right) .
$$

Now with these definitions at hand, we define the triangular Wulff functional $\mathcal{W}: \mathcal{C}^{1}(U) \rightarrow \mathbb{R}$ associated with the interaction potential $V$ by

$$
\mathcal{W}^{V}[v]:=\int_{U} \sigma_{\text {tilted }}^{V}\left(\frac{\partial v}{\partial r}, \frac{\partial v}{\partial \tau}\right) d r d \tau .
$$

Given a function defined on the bottom of the triangle $U$, we would like to study the minimum energy attained by a shape defined on $U$ agreeing with this function on the boundary. Namely, define the minimal Wulff functional $\mathcal{M}^{V}: \mathcal{C}^{1}([0,1]) \rightarrow \mathbb{R}$ by

$$
\mathcal{M}^{V}[\rho]:=\min _{v \in \mathcal{C}^{1}(U)_{\rho}} \mathcal{W}^{V}[v]=\min _{v \in \mathcal{C}^{1}(U)_{\rho}} \int_{U} \sigma_{\text {tilted }}^{V}\left(\frac{\partial v}{\partial r}, \frac{\partial v}{\partial \tau}\right) d r d \tau,
$$

where the minimisation is taken over $\mathcal{C}^{1}(U)_{\rho}:=\left\{v \in \mathcal{C}^{1}(U): v(r, 0)=\rho(r) \forall r \in[0,1]\right\}$.

We will be interested in functions $\rho:[0,1] \rightarrow \mathbb{R}$ minimising $\mathcal{M}^{V}[\rho]$ subject to certain costs. (Of course, here there are two different steps of minimisation taken place, first over internal values on the triangle $U$, and then on the boundary shape in $[0,1]$.) In any case, in order to study these sort of problems, we need a functional calculus allowing us to differentiate functionals. To this end, let $A: \mathcal{C}^{1}([0,1]) \rightarrow \mathbb{R}$ be a functional, and define the functional derivative $D_{A}$ of $A$ to be the map $D_{A}: \mathcal{C}^{1}([0,1]) \times \mathcal{C}^{1}([0,1]) \rightarrow \mathbb{R}$ given by

$$
D_{A}(\rho, \eta):=\left.\frac{d}{d u} A[\rho+u \eta]\right|_{u=0} .
$$


It is easily verified that $D_{A}$ is linear in the second argument for every $\rho$, and hence by the Riesz representation theorem [38], for every $\rho$ there exists a Radon measure $\Lambda_{A}(\rho, \cdot)$ on $[0,1]$ such that

$$
D_{A}(\rho, \eta)=\int_{0}^{1} \eta(r) \Lambda_{A}(\rho, d r)
$$

We call the measure $\Lambda_{A}(\rho, \cdot)$ the Riesz measure associated with $A$ and $\rho$. Note that whenever $\rho$ is a local minima or maxima of the functional $A$, the Riesz measure $\Lambda_{A}(\rho, \cdot)$ is zero. Let us also remark that whenever $A: \mathcal{C}^{1}([0,1]) \rightarrow \mathbb{R}$ is itself a linear map we have

$$
D_{A}(\rho, \eta)=A[\eta]
$$

Now to see an example of the functional derivative, consider the thermodynamic Vandermonde determinant

$$
\Delta[\rho]:=\int_{0<s<t<1} \log (\rho(s)-\rho(t))
$$

which was introduced in Sect. 4.6. A simple calculation taking the derivative inside the integral verifies that the Riesz measure associated with $\Delta$ has continuous density given by

$$
\Lambda_{\Delta}(\rho, r)=\int_{0}^{1} \frac{1}{\rho(r)-\rho(s)} d s .
$$

(That is, for measurable $\eta$ we have $\int \eta(r) \Lambda_{\Delta}(\rho, r) d r=\int \eta(r) \int_{0}^{1} \frac{1}{\rho(r)-\rho(s)} d s d r$.) We will be interested in studying the functional derivatives of minimal Wulff functionals. Namely, given a surface tension $V$, and a minimal Wulff functional $\mathcal{M}^{V}$, suppose that for a given $\rho$ the Riesz measure $\Lambda_{\mathcal{M}^{V}}(\rho, \cdot)$ has a continuous density which we denote by $\Lambda(\rho, r)$ for $r \in[0,1]$.

We now show that given $\rho$, the shape $v^{*}: U \rightarrow \mathbb{R}$ minimising the Wulff functional $\mathcal{W}^{V}$ subject to $v^{*}(r, 0)=\rho(r)$ must satisfying the differential equation

$$
\frac{\partial \sigma_{\text {tilted }}^{V}}{\partial q}\left(\rho^{\prime}(r), \frac{\partial v^{*}}{\partial \tau}(r, 0)\right)+\Lambda(\rho, r)=0 \quad \text { for } r \in[0,1] .
$$

Derivation of (8.8) Given a function $\rho:[0,1] \rightarrow \mathbb{R}$, consider breaking the minimisation procedure into two steps, by writing

$$
\min _{v \in \mathcal{C}^{1}(U)_{\rho}} \mathcal{W}^{V}[\rho]=\min _{f} \min _{v \in \mathcal{C}^{1}(U)_{\rho, f}} \mathcal{W}^{V}[v]
$$

where the former minimisation on the right hand side is taken over continuously differentiable functions $f:[0,1] \rightarrow \mathbb{R}$, and the latter minimisation on the right hand side is taken over $\min _{v \in \mathcal{C}^{1}(U)_{\rho, f}}$, the set of continuously differentiable $v: U \rightarrow \mathbb{R}$ satisfying

$$
v(r, 0)=\rho(r) \quad \text { and } \quad \frac{\partial v}{\partial \tau}(r, 0)=f(r) .
$$

Now given functions $\rho, f$, consider finding a $v$ minimising $\mathcal{W}^{V}[v]$ subject to the boundary conditions (8.10). For subsets $I$ of $[0,1]$, let $U_{I}:=U \cap\{\tau \in I\}$ and consider the disjoint union $U=U_{[0, h)} \cup U_{[h, 1]}$. Indeed, by separately considering the integration over the regions 
in this disjoint union, for small $h$ we have

$$
\begin{aligned}
& \min _{v \in \mathcal{C}^{1}(U)_{\rho, f}} \mathcal{W}^{V}[v] \\
& =o(h)+h \int_{0}^{1} \sigma_{\text {tilted }}\left(\rho^{\prime}(r), f(r)\right) d r+\min _{v \in \mathcal{C}^{1}\left(U_{[h, 1]}\right)_{\rho+h f}} \int_{U_{[h, 1]}} \sigma_{\text {tilted }}^{V}\left(\frac{\partial v}{\partial r}, \frac{\partial v}{\partial \tau}\right) d r d \tau,
\end{aligned}
$$

where $\mathcal{C}^{1}\left(U_{[h, 1]}\right)_{g}$ denotes the set of continuously differentiable functions defined on $U_{[h, 1]}$ and satisfying $v(h, r)=g(r)$ for all $-h / 2<r<1-h / 2$. For small $h$, it is easy to check from geometric considerations that

$$
\min _{v \in \mathcal{C}^{1}\left(U_{[h, 1]}\right)_{g}} \int_{U_{[h, 1]}} \sigma_{\text {tilted }}^{V}\left(\frac{\partial v}{\partial r}, \frac{\partial v}{\partial \tau}\right) d r d \tau=(1-2 h) \mathcal{M}^{V}[g]+o(h) .
$$

(This follows from the fact that $\operatorname{Leb}\left(U_{[h, 1]}\right)=(1-2 h+o(h)) \operatorname{Leb}(U)$, where Leb denotes the Lebesgue measure on $\mathbb{R}^{2}$.) In particular,

$$
\begin{aligned}
& \min _{\substack{v \in \mathcal{C}^{1}\left(U_{[h, 1]}\right)_{\rho+h f}\\
}} \int_{U_{[h, 1]}} \sigma_{\text {tilted }}^{V}\left(\frac{\partial v}{\partial r}, \frac{\partial v}{\partial \tau}\right) d r d \tau \\
& =\mathcal{M}^{V}[\rho]+h\left(-2 \mathcal{M}^{V}[\rho]+D_{\mathcal{M}^{V}}(\rho, f)\right)+o(h) .
\end{aligned}
$$

Plugging (8.13) into (8.11), we obtain

$$
\begin{aligned}
& \min _{v \in \mathcal{C}^{1}(U)_{\rho, f}} \mathcal{W}^{V}[v]:=\mathcal{M}^{V}[\rho] \\
& +h\left\{\int_{0}^{1} \sigma_{\text {tilted }}\left(\rho^{\prime}(r), f(r)\right) d r-2 \mathcal{M}^{V}[\rho]+D_{\mathcal{M}^{V}}(\rho, f)\right\}+o(h)
\end{aligned}
$$

Comparing (8.14) and (8.9), we see that the minimising function $f$ in (8.9) must minimise $\mathcal{G}_{\rho}[f]$, where

$$
\mathcal{G}_{\rho}[f]:=\int_{0}^{1} \sigma_{\text {tilted }}\left(\rho^{\prime}(r), f(r)\right) d r+D_{\mathcal{M}^{v}}(\rho, f) .
$$

Using our assumption that the Riesz derivative has a continuous density $\Lambda_{\mathcal{M}^{v}}(\rho, r)$, we may write

$$
\mathcal{G}_{\rho}[f]:=\int_{0}^{1}\left\{\sigma_{\text {tilted }}\left(\rho^{\prime}(r), f(r)\right)+\Lambda_{\mathcal{M}^{V}}(\rho, r) f(r)\right\} d r .
$$

The result now follows from a standard variational argument. Indeed, suppose $f^{*}$ is a minimiser of $\mathcal{G}_{\rho}[f]$. Then for every test function $\eta$ we have $\frac{d}{d s} \mathcal{G}_{\rho}\left[f^{*}+s \eta\right]=0$, which amounts to

$$
\int_{0}^{1}\left\{\frac{\partial \sigma_{\text {tilted }}}{\partial q}\left(\rho^{\prime}(r), f^{*}(r)\right)+\Lambda_{\mathcal{M}^{v}}(\rho, r)\right\} \eta(r) d r .
$$

In order for (8.15) to hold for every $\eta$, we must have $\frac{\partial \sigma_{\text {tilted }}}{\partial q}\left(\rho^{\prime}(r), f^{*}(r)\right)+\Lambda_{\mathcal{M}^{v}}(\rho, r)=0$. This equation must now be satisfied by $f^{*}(r):=\frac{\partial v^{*}}{\partial \tau}(r, 0)$, where $v^{*}$ is the minimiser of $\mathcal{W}^{V}[v]$ over $\mathcal{C}^{1}(U)_{\rho}$, completing the derivation of (8.8). 


\subsection{A Scaling Property of the Surface Tension}

The surface tension of the bead model satisfies a certain scaling property. Namely it is easily seen by setting $V(u)=\operatorname{bead}(u)$ in (4.1) that for all $s, t<0$, and $\lambda>0$, we have

$$
\sigma_{N}^{\text {bead }}(\lambda s, \lambda t)=-\log \lambda+\sigma_{N}^{\text {bead }}(s, t),
$$

for each of the finite surface tensions $\sigma_{N}^{\text {bead }}$. The limit $\sigma^{\text {bead }}(s, t):=\lim _{N \rightarrow \infty} \sigma_{N}^{\text {bead }}(s, t)$ inherits the same property, and using (8.3), so does the tilted surface tension:

$$
\sigma_{\text {tilted }}^{\text {bead }}(\lambda p, \lambda q)=-\log \lambda+\sigma_{\text {tilted }}^{\text {bead }}(p, q) \text {. }
$$

In particular, for $p<0$ by setting $\lambda=|p|$ in (8.17), we see that there is a function $\Omega$ such that

$$
\sigma_{\text {tilted }}^{\text {bead }}(p, q)=-\log (|p|)+\Omega(q /|p|) .
$$

We remark that in particular,

$$
\frac{\partial \sigma_{\text {tilted }}^{V}}{\partial q}(p, q)=\frac{1}{|p|} \Omega^{\prime}(q /|p|) .
$$

\subsection{Derivation of Equation 4.21}

Recall now that the goal of this section is to derive the formula

$$
\sigma_{\text {tilted }}^{\text {bead }}(p, q):= \begin{cases}C-\log \left(|p| \cos \left(\pi \frac{q}{|p|}\right)\right) & \text { if } p<0,|q|<\frac{1}{2}|p| \\ \infty & \text { otherwise, }\end{cases}
$$

for the surface tension of the bead model. First, we remark that whenever we do not have $p<0$ and $q<|p|$, we have $\sigma_{\text {tilted }}^{\text {bead }}(p, q):=\infty$. Indeed, it is immediate from the definition that for the finite (untilted) surface tension we have $\sigma_{N}^{\text {bead }}(s, t)<\infty$ if and only if both $s$ and $t$ are strictly negative. In particular, $\sigma^{\text {bead }}(s, t):=\lim _{N \rightarrow \infty} \sigma_{N}^{\text {bead }}(s, t)$ also inherits this property. Now setting $s=\frac{1}{2} p-q$ and $t=\frac{1}{2} p+q$, we see that $(s<0, t<0)$ is equivalent to $\left(p<0,|q|<\frac{1}{2}|p|\right)$. In particular,

$$
\sigma_{\text {tilted }}^{\text {bead }}(p, q)<\infty \quad \text { only if } \quad p<0,|q|<\frac{1}{2}|p| .
$$

It remains to actually compute $\sigma_{\text {tilted }}^{\text {bead }}(p, q)$ in the case that $p<0$ and $|q|<|p|$.

Clearly, by (8.18), (8.19) and (8.21), in order to establish (8.20) it is remains to show that

$$
\Omega^{\prime}(s)=\pi \tan \pi s \quad \text { for } s \in(-1 / 2,1 / 2) .
$$

To this end, recall that in Sect. 4.6 we introduced the semicircle law as the minimiser of a variational problem. We now restate this problem here in terms of the tilted coordinates system. Namely given $x \in[0,1]$, we recall that $\rho_{\mathrm{sc}}(x)$ is the solution in $[-2,2]$ to the equation

$$
\int_{\rho_{\mathrm{sc}}(x)}^{2} \frac{1}{2 \pi} \sqrt{4-u^{2}} d u=x .
$$


Now consider the function $\xi_{s c}$ in (4.22) defined in the tilted change of coordinates (8.1), namely

$$
\xi_{\mathrm{sC}}(r, \tau):=\sqrt{1-\tau} \rho_{\mathrm{sC}}\left(\frac{r-\tau / 2}{1-\tau}\right) .
$$

Let $K:=\{(r, \tau): \tau \in[-1,1],|\tau| / 2<r<1-|\tau| / 2\}$ be the diamond in $\mathbb{R}^{2}$. Restating the variational characterisation of $\xi_{\mathrm{sc}}$ in Sect. 4.6 here in tilted coordinates, the function $\xi_{\mathrm{sc}}$ is the minimiser over $v: K \rightarrow \mathbb{R}$ of the functional

$$
E[v]:=\int_{K} \sigma_{\text {tilted }}^{V}\left(\frac{\partial v}{\partial r}, \frac{\partial v}{\partial \tau}\right) d r d \tau+\frac{1}{2} \int_{0}^{1} v(r, 0)^{2} d r .
$$

Equivalently, the restriction of $\xi_{\mathrm{sc}}$ to $U=\{(r, \tau): \tau \in[0,1], \tau / 2<r<1-\tau / 2\}$ is the minimiser of

$$
E^{\prime}[v]:=2 \int_{U} \sigma_{\text {tilted }}^{V}\left(\frac{\partial v}{\partial r}, \frac{\partial v}{\partial \tau}\right) d r d \tau+\frac{1}{2} \int_{0}^{1} v(r, 0)^{2} d r .
$$

Note that $\xi_{\mathrm{sc}}(r, 0)=\rho_{\mathrm{sc}}(r)$. In particular, breaking the minimisation of $E^{\prime}[v]$ into two steps, we see that the function $\xi_{\mathrm{sc}}(r, \tau)$ is the minimiser of $\int_{U} \sigma_{\text {tilted }}^{V}\left(\frac{\partial v}{\partial r}, \frac{\partial v}{\partial \tau}\right) d r d \tau$ over $\mathcal{C}^{1}(U)_{\rho_{\mathrm{sc}}}$. It follows that by (8.8), we must have

$$
\frac{\partial \sigma_{\text {tilted }}^{V}}{\partial q}\left(\rho_{\mathrm{sc}}^{\prime}(r), \frac{\partial \xi_{\mathrm{sc}}}{\partial \tau}(0, r)\right)+\Lambda^{\text {bead }}\left(\rho_{\mathrm{sc}}, r\right)=0 \quad \text { for every } r \in[0,1] .
$$

Using the fact that the Eq. (8.25) involves each of the known quantities $\rho_{\mathrm{sc}}$, given in (8.23), $\xi_{\text {sc }}$ given in (8.24) and $\Lambda^{\text {bead }}$ given in (8.7), along with the representation (8.18), this is sufficient to establish (8.22).

Indeed, as a first step, using (8.19) and (8.7) we may rewrite (8.25) as

$$
\frac{1}{\rho_{\mathrm{SC}}^{\prime}(r)} \Omega^{\prime}\left(\frac{\frac{\partial \xi_{\mathrm{SC}}}{\partial \tau}(r, 0)}{\rho_{\mathrm{SC}}^{\prime}(r)}\right)+\int_{0}^{1} \frac{1}{\rho_{\mathrm{SC}}(r)-\rho_{\mathrm{SC}}(s)} d s=0 \quad \text { for every } r \in[0,1] \text {. }
$$

It turns out to be easiest to consider Eq. (8.26) through the change of variable $\rho_{\mathrm{sc}}(r)=$ $2 \sin \phi$. Now some computations are required. Let $f_{\mathrm{sc}}(x)=\frac{1}{2 \pi} \sqrt{4-x^{2}} \mathbb{1}_{x \in[-2,2]}$, and let $F_{\mathrm{SC}}(x):=\int_{-\infty}^{x} f(u) d u$. Then $\rho_{\mathrm{sc}}$ is the inverse function of $1-F$. Straightforward geometric considerations tell us that for $\phi \in[-\pi / 2, \pi / 2]$,

$$
F_{\mathrm{sC}}(2 \sin \phi)=\frac{1}{2}+\frac{\phi}{\pi}+\frac{\sin \phi \cos \phi}{\pi} .
$$

Using the inverse function theorem to obtain the first inequality below, it follows that with $\rho_{\mathrm{sc}}(r)=2 \sin \phi$ we have

$$
\rho_{\mathrm{sc}}^{\prime}(r)=-\frac{1}{F_{\mathrm{sc}}^{\prime}\left(\rho_{\mathrm{sc}}(r)\right)}=-\frac{1}{\frac{1}{2 \pi} \sqrt{4-(2 \sin \phi)^{2}}}=-\frac{\pi}{\cos \phi} .
$$

A computation shows that

$$
\frac{\partial \xi_{\mathrm{sc}}}{\partial \tau}(\tau, r)=\frac{-1 / 2}{(1-\tau)^{1 / 2}} \rho_{\mathrm{sc}}\left(\frac{r-\tau / 2}{1-\tau}\right)+\left(\frac{r-\tau / 2}{(1-\tau)^{3 / 2}}-\frac{1 / 2}{(1-\tau)^{1 / 2}}\right) \rho_{\mathrm{sc}}^{\prime}\left(\frac{r-\tau / 2}{1-\tau}\right),
$$

and in particular, on the line $\tau=0$ we have

$$
\frac{\partial \xi_{\mathrm{sc}}}{\partial \tau}(r, \tau)=-\frac{1}{2} \rho_{\mathrm{sc}}(r)+\left(r-\frac{1}{2}\right) \rho_{\mathrm{sc}}^{\prime}(r) .
$$


Now note that if $\rho_{\mathrm{SC}}(r)=2 \sin \phi$, then $r=1-F_{\mathrm{SC}}\left(\rho_{\mathrm{SC}}(r)\right)=\frac{1}{2}-\frac{\phi}{\pi}-\frac{\sin \phi \cos \phi}{\pi}$. Thus using (8.27), (8.28) reduces to

$$
\frac{\partial \xi_{\mathrm{sc}}}{\partial \tau}(r, \tau)=\frac{-1}{2} 2 \sin \phi+\left(\frac{\phi}{\pi}+\frac{\sin \phi \cos \phi}{\pi}\right) \frac{\pi}{\cos \phi}=\frac{\phi}{\cos \phi} .
$$

We are now ready to show that Eq. (8.26) implies (8.22). On the one hand, by (8.27) and (8.29) with $\rho_{\mathrm{sc}}(r)=2 \sin \phi$ we have

$$
\frac{1}{\rho_{\mathrm{SC}}^{\prime}(r)} \Omega^{\prime}\left(\frac{\frac{\partial \xi_{\mathrm{sc}}}{\partial \tau}(r, 0)}{\rho_{\mathrm{sc}}^{\prime}(r)}\right)=-\frac{\cos \phi}{\pi} \Omega^{\prime}(-\phi / \pi) .
$$

On the other hand, we now claim that thanks to a special property of the semicircle law, we have

$$
\int_{0}^{1} \frac{1}{\rho_{\mathrm{sc}}(r)-\rho_{\mathrm{sC}}(s)} d s=\frac{1}{2} \rho_{\mathrm{sC}}(r)=\sin \phi .
$$

Indeed, by changing variable at the point $\rho_{\mathrm{sc}}(r)=2 \sin \phi$, we have

$$
\int_{0}^{1} \frac{1}{\rho_{\mathrm{SC}}(r)-\rho_{\mathrm{SC}}(s)} d s=-\int_{-2}^{2} \frac{1}{2 \sin \phi-u} \frac{1}{2 \pi} \sqrt{4-u^{2}} d u .
$$

It is now a property of the semicircle law well known in random matrix theory (see e.g. [31, Eq. 4.2.7]) that for $\alpha \in[-2,2]$ we have $\int_{-2}^{2} \frac{1}{\alpha-u} \frac{1}{2 \pi} \sqrt{4-u^{2}} d u=\frac{1}{2} \alpha$, and hence by (8.32) we have

$$
\int_{0}^{1} \frac{1}{\rho_{\mathrm{sC}}(r)-\rho_{\mathrm{SC}}(s)} d s=-\sin \phi
$$

(where $\phi$ is implicit in the equation $\rho_{\mathrm{sC}}(r)=2 \sin \phi$ ).

Plugging (8.30) and (8.33) into (8.26), we obtain

$$
\Omega^{\prime}(-\phi / \pi)=-\pi \tan \phi,
$$

which implies that $\Omega^{\prime}(s)=\pi \tan \pi s$, proving (8.22), and therefore the formula (4.21).

Acknowledgements Open access funding provided by University of Graz. The authors are extremely grateful to an anonymous referee whose suggestions have greatly improved this article. Research supported by the European Research Council (Grant No. 669306).

Open Access This article is licensed under a Creative Commons Attribution 4.0 International License, which permits use, sharing, adaptation, distribution and reproduction in any medium or format, as long as you give appropriate credit to the original author(s) and the source, provide a link to the Creative Commons licence, and indicate if changes were made. The images or other third party material in this article are included in the article's Creative Commons licence, unless indicated otherwise in a credit line to the material. If material is not included in the article's Creative Commons licence and your intended use is not permitted by statutory regulation or exceeds the permitted use, you will need to obtain permission directly from the copyright holder. To view a copy of this licence, visit http://creativecommons.org/licenses/by/4.0/.

\section{References}

1. Anderson, G., Guionnet, A., Zeitouni, O.: A Introduction to Random Matrices. Cambridge Studies in Advanced Mathematics. Cambridge University Press, Cambridge (2009)

2. Barraquand, G., Corwin, I.: Random-walk in beta-distributed random environment. Probab. Theor. Relat. Fields 167(3), 1057-1116 (2017) 
3. Baryshnikov, Y.: GUEs and queues. Probab. Theor. Relat. Fields 119, 256-274 (2001)

4. Boutillier, C.: The bead model and limit behaviours of dimer models. Ann. Probab. 37(1), 107-142 (2009)

5. Böttcher, A., Silbermann, B.: Introduction to Large Truncated Toeplitz Matrices. Universitext. Springer, New York (1999)

6. Bump, D.: Lie Groups. GTM, 2nd edn. Springer, New York (2013)

7. Chen, C.-P.: Asymptotic expansions for superfactorial. J. Number. Theory 135, 36-42 (2014)

8. Cohn, H., Larson, M., Propp, J.: The shape of a typical boxed plane partition New York. J. Math. 4, 137-165 (1998)

9. Cohn, H., Kenyon, R., Propp, J.: A variational principle for domino tilings. J. Am. Math. Soc. 14(2), 297-346 (2000)

10. Comets, F.: Directed Polymers in Random Environments. École d'Éte de Probabilités de Saint-Flour XLVI. Springer, New York (2016)

11. Corwin, I., Hammond, A.: Brownian Gibbs property for Airy line ensembles. Invent. Math. 195, 441-508 (2014)

12. Corwin, I., Hammond, A.: KPZ Line ensemble. Probab. Theory Relat. Field. 166, 67-185 (2015)

13. Corwin, I., Nica, M.: Intermediate disorder directed polymers and the multi-layer extension of the stochastic heat equation. Electron. J. Probab. 22, 49 (2017)

14. Corwin, I., O’Connell, N., Seppäläinen, T., Zygouras, N.: Tropical combinatorics and Whittaker functions. Duke Math. J. 163(3), 513-563 (2014)

15. Corwin, I., Seppäläinen, T., Shen, H.: The strict-weak lattice polymer. J. Stat. Phys. 160(4), 1027-1053 (2014)

16. Dauvergne, D., Orthmann, J., Virág, B.: The directed landscape (2018). arXiv:1812.00309

17. Doumerc, Y.: A note on representations of eigenvalues of classical Gaussian matrices. Seminaire de Probabilités XXXVII. Lecture Notes in Mathematics, vol. 1832, pp. 370-384. Springer, Berlin (2003)

18. Forrester, P.J.: Log-Gases and Random Matrices. Princeton University Press, Princeton (2010)

19. Funaki, T.: Stochastic Interface Models. École d'Éte de Probabilités de Saint-Flour XXXIII. Lectures on Probability Theory and Statistics. Springer, New York (2003)

20. Funaki, T., Spohn, H.: Motion by mean curvature from the Ginzburg-Landau $\nabla \phi$ interface model. Commun. Math. Phys. 185, 1-36 (1997)

21. Glynn, P.W., Whitt, W.: Departures from many queues in series. Ann. Appl. Probab. 1(4), 546-572 (1991)

22. Johansson, K.: Shape fluctuations and random matrices. Commun. Math. Phys. 209(2), 437-476 (2000)

23. Johansson, K.: Non-intersecting, simple, symmetric random walks and the extended Hahn kernel. Ann. Inst. Fourier 55(6), 2129-2145 (2005)

24. Johannson, K., Nordenstam, E.: Eigenvalues of GUE minors. Electron. J. Probab. 11, 1342-1371 (2006)

25. Kenyon, R.: Lectures on dimers (2009). arXiv:0910.3129

26. Kingman, J.F.C.: Subadditive ergodic theory. Ann. Probab. 1(6), 883-899 (1973)

27. Krattenthaler, C.: Advanced determinant calculus. In: Foata, D., Han, G.N. (eds.) The Andrews Festschrift. Springer, Heidelberg (2001)

28. Liggett, T.M.: An improved subadditive ergodic theorem. Ann. Probab. 13(4), 1278-1285 (1985)

29. Macdonald, I.G.: Symmetric Functions and Hall Polynomials, 2nd edn. Oxford University Press Inc., New York (1995)

30. Metcalfe, T.: Universality properties of Gelfand-Tsetlin patterns. Probab. Theor. Relat. Fields 155, 303346 (2013)

31. Mehta, M.L.: Random Matrices, 3rd edn. Elsevier, Amsterdam (2004)

32. Moriarty, J., O'Connell, N.: On the free energy of a directed polymer in a Brownian environment. Markov Process. Relat. 13(2), 251-266 (2007)

33. O'Connell, N.: Directed percolation and tandem queues. HPL-BRIMS-2000-28 (2000)

34. O'Connell, N.: Directed polymers and the quantum Toda lattice. Ann. Probab. 40(2), 437-458 (2012)

35. O'Connell, N., Ortmann, J.: Tracy-Widom asymptotics for a random polymer model with gammadistributed weights. Electron. J. Probab. 20, 25 (2015)

36. O'Connell, N., Yor, M.: Brownian analogues of Burke's theorem. Stoch. Proc. Appl. 96, 285-304 (2001)

37. O'Connell, N., Seppäläinen, T., Zygouras, N.: Geometric RSK correspondence, Whittaker functions and symmetrized random polymers. Invent. Math. 197, 361-416 (2014)

38. Riesz, F.: Sur les opérations fonctionnelles linéaires. C. R. Acad. Sci. Paris. 149, 974-977 (1909)

39. Rost, H.: Non-equilibrium behaviour of a many particle process: density profile and local equilibria. Z. Wahrsch. Verw. Gebeite 58, 41-53 (1981)

40. Seppäläinen, T.: Scaling limit for a one-dimensional directed polymer with boundary conditions. Ann. Probab. 40(1), 19-73 (2012)

41. Sheffield, S.: Random surfaces. Astérisque 304, 175 (2005)

42. Stanley, R.P.: Enumerative Combinatorics, vol. 2. Cambridge University Press, Cambridge (1999) 
43. Sun, W.: Dimer model, bead model and standard Young tableaux: finite cases and limit shapes (2018). arXiv: 1804.03414

44. Wu, X.: Tightness of discrete Gibbsian line ensembles with exponential interaction hamiltonians (2019). arXiv: 1909.00946

Publisher's Note Springer Nature remains neutral with regard to jurisdictional claims in published maps and institutional affiliations. 